\title{
Modular symmetry origin of texture zeros and quark-lepton unification
}

\author{
Jun-Nan Lu๑, ${ }^{1,2, *}$ Xiang-Gan Liu, ${ }^{1, \dagger}$ and Gui-Jun Ding $\oplus^{1, \hbar}$ \\ ${ }^{1}$ Interdisciplinary Center for Theoretical Study and Department of Modern Physics, \\ University of Science and Technology of China, Hefei, Anhui 230026, China \\ ${ }^{2}$ AHEP Group, Institut de Física Corpuscular-CSIC/Universitat de València, \\ Parc Científic de Paterna. C/ Catedrático José Beltrán, 2 E-46980 Paterna (Valencia), Spain
}

(Received 22 January 2020; accepted 17 May 2020; published 17 June 2020)

\begin{abstract}
The even-weight modular forms of level $N$ can be arranged into the common irreducible representations of the inhomogeneous finite modular group $\Gamma_{N}$ and the homogeneous finite modular group $\Gamma_{N}^{\prime}$ which is the double covering of $\Gamma_{N}$, and the odd-weight modular forms of level $N$ transform in the new representations of $\Gamma_{N}^{\prime}$. We find that the above structure of modular forms can naturally generate texture zeros of the fermion mass matrices if we properly assign the representations and weights of the matter fields under the modular group. We perform a comprehensive analysis for the $\Gamma_{3}^{\prime} \cong T^{\prime}$ modular symmetry. The three generations of left-handed quarks are assumed to transform as a doublet and a singlet of $T^{\prime}$, and we find six possible texture-zero structures of the quark mass matrix up to row and column permutations. We present five benchmark quark models which can produce very good fits to the experimental data. These quark models are further extended to include the lepton sector, and the resulting models can give a unified description of both quark and lepton masses and flavor mixing simultaneously, although they contain a smaller number of free parameters than the observables.
\end{abstract}

DOI: $10.1103 /$ PhysRevD.101.115020

\section{INTRODUCTION}

The standard model (SM) of particle physics has been extensively tested. However, neither significant evidence of departures from the SM nor convincing hints for the presence of new physics have been found. The masses of quarks and charged leptons are parametrized by the Yukawa coupling constants in the SM. The Yukawa sector is still poorly understood, and the SM itself cannot predict the exact values of quark masses and the CabibboKobayashi-Maskawa (CKM) mixing matrix. The fundamental principle that determines the hierarchical charged fermion mass spectra, tiny neutrino masses, flavor mixing, and $C P$ violation is still elusive. Neutrino oscillation experiments have made enormous progress in recent years. The three lepton mixing angles and neutrino mass-squared differences have been precisely measured. The latest global fit of neutrino oscillation data gives the best-fit values and $1 \sigma$ errors of the lepton mixing angles as $\theta_{12} /{ }^{\circ}=33.82_{-0.76}^{+0.78}$,

\footnotetext{
hitman@mail.ustc.edu.cn

†hepliuxg@mail.ustc.edu.cn

\#dinggj@ustc.edu.cn
}

Published by the American Physical Society under the terms of the Creative Commons Attribution 4.0 International license. Further distribution of this work must maintain attribution to the author(s) and the published article's title, journal citation, and DOI. Funded by SCOAP. $\theta_{23} /^{\circ}=49.6_{-1.2}^{+1.0}$, and $\theta_{13} /^{\circ}=8.61 \pm 0.13$ [1]. The present neutrino oscillation data favor normal ordering for the neutrino mass spectrum over inverted ordering, $C P$ conservation in neutrino oscillation is disfavored at the $2 \sigma$ confidence level, and a value of the leptonic Dirac $C P$ phase $\delta_{C P}$ around $3 \pi / 2$ is preferred [2,3].

Neutrino oscillation provides us new insights into understanding the flavor puzzle. It was found that the neutrino mixing angles can be reproduced by extending the SM with a finite discrete non-Abelian flavor symmetry [4-10]. The observed neutrino mixing pattern arises as the result of a particular vacuum alignment of scalar fields called flavons, which spontaneously break certain discrete flavor symmetries. The flavons are SM singlets and they transform nontrivially under the flavor symmetry group. Usually a number of flavons are necessary, and the scalar potential as well as additional shaping symmetries have to be cleverly designed to obtain the desired vacuum alignment. Another drawback of this approach is that the predictability of a discrete flavor symmetry model could be degraded by possible higher-dimensional operators. Moreover, flavor symmetry is usually used to constrain the neutrino mixing angles, while the neutrino masses are undetermined except in some specific models.

In order to overcome the above-mentioned drawbacks of the conventional finite discrete flavor symmetry, a new approach where modular invariance plays the role of flavor symmetry was recently proposed in Ref. [11]. Modular 
invariance has a long history in both string and field theories. The idea of modular invariance as a flavor symmetry to constrain the Yukawa couplings has been naturally realized in string theory [12-14], $D$-brane compactification $[15,16]$, magnetized extra dimensions $[17,18]$, and orbifold compactification [19,20]. Modular invariance has been used to address several aspects of the flavor problem in model building [21-23]. In the present paper, we follow Ref. [11] and take a bottom-up approach based on supersymmetric modular invariant theories. In the most economical version of the modular invariant models, the flavon fields other than the modulus are not needed and the flavor symmetry is uniquely broken by the vacuum expectation value (VEV) of the complex modulus $\tau$. The complicated vacuum alignment is not required, although one needs some mechanism to fix the value of $\tau$. The Yukawa couplings are functions of modular forms which are holomorphic functions of $\tau$, and all higher-dimensional operators in the superpotential are completely determined by modular invariance in the limit of supersymmetry [11]. It is notable that the neutrino mass models based on modular invariance could involve only a few coupling constants such that neutrino masses and mixing parameters are correlated. This formalism has been extended to consistently combine with the generalized $C P$ symmetry, and the consistency conditions require that the modulus transforms as $\tau \rightarrow-\tau^{*}$ up to modular transformations under the action of $C P$ symmetry [24-29]. In a symmetric basis where the representation matrices of both $S$ and $T$ are symmetric, the multiplets of modular forms become complex conjugated under $C P$ transformation if they are properly normalized. As a consequence, the generalized $C P$ symmetry would constrain all of the couplings in a modular-invariant model to be real [24], and thus the predictive power of such models is enhanced. It was noted that the Kähler potential is not fixed by the symmetries and transformation properties of the models, although the superpotential is completely fixed by the modular transformations. The corrections from the most general Kähler potential consistent with the symmetries of the model could potentially reduce the predictive power of this formalism [30].

The crucial element of this new approach is the modular forms of even weight and level $N$ which can be arranged into irreducible representations of the inhomogeneous finite modular group $\Gamma_{N}$. Several models of lepton masses and mixing have been constructed based on the finite modular groups $\Gamma_{2} \cong S_{3}$ [31-34], $\Gamma_{3} \cong A_{4}[11,31,32,35-$ $51], \Gamma_{4} \cong S_{4}[48,52-58]$, and $\Gamma_{5} \cong A_{5}[57,59,60]$. This new approach has been extended to modular forms of general integer weight which can be arranged into irreducible representations of the homogeneous finite modular group $\Gamma_{N}^{\prime}$ [61]. Notice that $\Gamma_{N}^{\prime}$ is the double covering of $\Gamma_{N}$. The modular forms of weight 1 and level 3 have been explicitly constructed and shown to furnish a two-dimensional irreducible representation of $\Gamma_{3}^{\prime} \cong T^{\prime}$ [61]. The phenomenological predictions of modular symmetry models for leptogenesis have been discussed in Refs. [47,58]. The $\mathrm{SU}(5)$ grand unified models with modular symmetry have been constructed $[33,37]$. The modular symmetry has also been applied to dark matter and radiative neutrino mass models $[40,44,45]$.

An interesting attempt to understand the dynamics of fermion mass generation and flavor mixing is to assume some entries of the fermion mass matrices are vanishing [62-64], and such scenarios are more popularly known as texture-zero models. Usually, Abelian flavor symmetry is used to exactly or approximately realize texture-zero structures $[65,66]$. Systematic and complete studies of all possibilities have been performed for both the lepton sector [67] and quark sector [68]. For recent reviews on texture zeros we refer the reader to Refs. [69,70]. The homogeneous finite modular group $\Gamma_{N}^{\prime}$ is the double covering of the inhomogeneous finite modular group $\Gamma_{N}$, and $\Gamma_{N}^{\prime}$ has twice as many elements as $\Gamma_{N}$. Besides the irreducible representations of $\Gamma_{N}, \Gamma_{N}^{\prime}$ has other new representations [61]. The even-weight modular forms of level $N$ can be arranged into the irreducible representations of $\Gamma_{N}$ up to the automorphy factor [11], while the odd-weight modular forms of level $N$ are arranged into the new representations of $\Gamma_{N}^{\prime}$ [61]. In this work, we show that the above structure of modular forms can naturally produce texture zeros of the fermion mass matrices if we properly assign the representations and weights of the matter fields under the modular group. In this sense, the modular invariance approach has the merits of both Abelian flavor symmetry and discrete non-Abelian flavor symmetry.

In the present paper, we use the modular forms of level 3 to show concrete examples. In addition to the representations $\mathbf{1}, \mathbf{1}^{\prime}, \mathbf{1}^{\prime \prime}$, and $\mathbf{3}$ of $\Gamma_{3} \cong A_{4}, \Gamma_{3}^{\prime} \cong T^{\prime}$ has three doublet representations: $\mathbf{2}, \mathbf{2}^{\prime}$, and $\mathbf{2}^{\prime \prime}$. The even-weight modular forms of level 3 transform as singlets and triplets of $\Gamma_{3}^{\prime}$, while the odd-weight modular forms of level 3 are arranged into $\Gamma_{3}^{\prime}$ doublets. We perform a comprehensive analysis of possible texture zeros of the quark mass matrices with the introduction of the $T^{\prime}$ modular symmetry. Since the mass of the third-generation quark is much heavier than the first two generations, we assume that the three generations of lefthanded quark fields transform as a doublet and a singlet under $T^{\prime}$ modular symmetry, while the right-handed quark fields can be assigned to a direct sum of a doublet and singlet of $T^{\prime}$, or they could be three singlets of $T^{\prime}$.

This paper is structured as follows. In Sec. II we briefly review the basic aspects of modular symmetry and give the expressions of the modular forms of level 3. In Sec. III we present the possible structures and texture zeros of the quark mass matrices if both left-handed and righthanded quark fields are assigned to a direct sum of singlet and doublet representations of $T^{\prime}$. The texture-zero structures of the quark mass matrices for singlet assignments of 
right-handed quark fields are given in Sec. IV. Furthermore, in Sec. V we give five benchmark quark models, which lead to up- and down-quark mass matrices with texture zeros. These models contain only 10 or 11 independent real parameters, and they produce excellent fits to the data on quark masses and the CKM mixing matrix. In Sec. VI we include the lepton sector to give a unified description of both quark and lepton masses and flavor mixing simultaneously. The nonminimal Kähler potential and its contribution in the quark sector are discussed in Sec. VII, and we give the most general form of the Kähler potential consistent with modular symmetry. Finally, we conclude and draw our conclusions in Sec. VIII. We give the representation matrices of the generators of the $T^{\prime}$ group and the Clebsch-Gordan (CG) coefficients in our basis in Appendix A. The modular forms of higher weights $k=5$, 6, 7, 8 and level 3 are collected in Appendix B. The nonminimal Kähler potential in the lepton sector is discussed in Appendix C.

\section{MODULAR SYMMETRY AND MODULAR FORMS OF LEVEL $N=3$}

The modular group $\bar{\Gamma}$ can be regarded as the group of linear fraction transformations acting on the complex modulus $\tau$ with $\operatorname{Im} \tau>0$,

$\tau \rightarrow \gamma \tau=\frac{a \tau+b}{c \tau+d}, \quad a, b, c, d \in \mathbb{Z}, \quad a d-b c=1$.

Obviously, the linear fraction transformation $\frac{a \tau+b}{c \tau+d}$ is identical to $\frac{-a \tau-b}{-c \tau-d}$. Therefore, the modular group $\bar{\Gamma}$ is isomorphic to $\operatorname{PSL}(2, \mathbb{Z})=S L(2, Z) /\{I,-I\}$, where $S L(2, \mathbb{Z})$ is the group of $2 \times 2$ matrices with integer entries and determinant 1 , and $I$ refers to the two-dimensional unit matrix. The modular group has infinite elements, and it can be generated by two transformations $S$ and $T$,

$$
S: \tau \rightarrow-\frac{1}{\tau}, \quad T: \tau \rightarrow \tau+1,
$$

which fulfill the relations

$$
S^{2}=(S T)^{3}=1
$$

and $(T S)^{3}=1$, which is equivalent to $(S T)^{3}=1$ if $S^{2}=1$. Let $N$ be a positive integer. The principal congruence subgroup of level $N$ is

$$
\begin{aligned}
\Gamma(N) & =\left\{\left(\begin{array}{ll}
a & b \\
c & d
\end{array}\right) \in S L(2, \mathbb{Z}),\left(\begin{array}{ll}
a & b \\
c & d
\end{array}\right)\right. \\
& \left.=\left(\begin{array}{ll}
1 & 0 \\
0 & 1
\end{array}\right)(\bmod \mathrm{N})\right\},
\end{aligned}
$$

which is an infinite normal subgroup of $S L(2, \mathbb{Z})$. It is obvious that $\Gamma(1) \cong S L(2, \mathbb{Z})$. We define $\bar{\Gamma}(N)=$ $\Gamma(N) /\{I,-I\}$ for $N=1,2$ and $\bar{\Gamma}(N)=\Gamma(N)$ for $N>2$ since the element $-I$ does not belong to $\Gamma(N)$ for $N>2$. Note that $\bar{\Gamma}(1) \cong \operatorname{PSL}(2, \mathbb{Z}) \equiv \bar{\Gamma}$. The inhomogeneous finite modular groups are defined as the quotient groups $\Gamma_{N} \equiv \bar{\Gamma} / \bar{\Gamma}(N)$. Because the element $T^{N}$ belongs to $\Gamma(N)$, the finite modular group $\Gamma_{N}$ can be generated by $S$ and $T$, which obey the relations [71]

$$
S^{2}=(S T)^{3}=T^{N}=1 .
$$

The groups $\Gamma_{N}$ for $N=2,3,4,5$ are isomorphic to $S_{3}, A_{4}$, $S_{4}$, and $A_{5}$, respectively. Additional relations besides those in Eq. (5) are necessary in order to render the group finite for $N>5$. The homogeneous finite modular groups are the quotient groups $\Gamma_{N}^{\prime} \equiv S L(2, \mathbb{Z}) / \Gamma(N)$, and they can be generated by three generators $S, T$, and $\mathbb{R}$, which satisfy [61]

$S^{2}=\mathbb{R}, \quad(S T)^{3}=T^{N}=\mathbb{R}^{2}=1, \quad \mathbb{R} T=T \mathbb{R}$,

and additional constraints should be imposed for $N>5$. The group $\Gamma_{2}^{\prime}$ is isomorphic to $S_{3}$, and $\Gamma_{N}^{\prime}$ is the double covering of $\Gamma_{N}$ and it has twice as many elements as $\Gamma_{N}$. In the present work, we focus on $\Gamma_{3}^{\prime}$ which is the double covering of $A_{4}$ and is isomorphic to the binary tetrahedral group $T^{\prime}$.

Modular forms of weight $k$ and level $N$ are holomorphic functions $f(\tau)$, which transforms under the action of $\Gamma(N)$ in the following way:

$f(\gamma \tau)=(c \tau+d)^{k} f(\tau), \quad \gamma=\left(\begin{array}{ll}a & b \\ c & d\end{array}\right) \in \Gamma(N)$,

where $k$ is a generic non-negative integer. The modular forms of weight $k$ and level $N$ span a linear space of finite dimension. As shown in Ref. [61], the modular forms can be organized into some modular multiplets $f_{\mathbf{r}} \equiv$ $\left(f_{1}(\tau), f_{2}(\tau), \ldots\right)^{T}$ which transform as a certain irreducible representation $\mathbf{r}$ of the finite modular group $\Gamma_{N}^{\prime}[11,61]$, i.e.,

$f_{\mathbf{r}}(\gamma \tau)=(c \tau+d)^{k} \rho_{\mathbf{r}}(\gamma) f_{\mathbf{r}}(\tau) \quad$ for $\quad \forall \gamma \in S L(2, \mathbb{Z})$,

where $\gamma$ is the representative element of the $\operatorname{coset} \gamma \Gamma(N)$ in $\Gamma_{N}^{\prime}$, and $\rho_{\mathbf{r}}(\gamma)$ is the representation matrix of the element $\gamma$ in the irreducible representation $\mathbf{r}$.

\section{A. Modular forms of level 3}

The linear space of modular forms of weight $k$ and level 3 has dimension $k+1$. The modular forms of level 3 have been constructed in terms of the Dedekind eta function $\eta(\tau)$ [61]. There are two linearly independent modular forms of the lowest weight 1 with $k=1$, and they can be arranged into a doublet $\mathbf{2}$ of $T^{\prime}$, 


$$
\begin{aligned}
Y_{2}^{(1)}(\tau) & \equiv\left(\begin{array}{c}
Y_{2,1}^{(1)}(\tau) \\
Y_{2,2}^{(1)}(\tau)
\end{array}\right) \\
& =\left(\sqrt{2} e^{i 7 \pi / 12} \frac{\eta^{3}(3 \tau)}{\eta(\tau)}, \frac{\eta^{3}(3 \tau)}{\eta(\tau)}-\frac{1}{3} \frac{\eta^{3}(\tau / 3)}{\eta(\tau)}\right)^{T} .
\end{aligned}
$$

Note that the overall coefficient of $Y_{2}^{(1)}$ cannot be uniquely determined. We also use the notation $Y_{\mathbf{2}, 1}^{(1)}(\tau)=Y_{1}(\tau)$ and $Y_{\mathbf{2}, 2}^{(1)}(\tau)=Y_{2}(\tau)$ for simplicity in the following. The doublet modular forms $Y_{\mathbf{2}, 1}^{(1)}$ and $Y_{\mathbf{2}, 2}^{(1)}$ have the following $q$ expansions:

$$
\begin{aligned}
Y_{2,1}^{(1)}(\tau)= & \sqrt{2} e^{7 \pi i / 12} q^{1 / 3}\left(1+q+2 q^{2}+2 q^{4}+q^{5}\right. \\
& \left.+2 q^{6}+q^{8}+2 q^{9}+2 q^{10}+\cdots\right), \\
Y_{2,2}^{(1)}(\tau)= & 1 / 3+2 q+2 q^{3}+2 q^{4}+4 q^{7}+2 q^{9} \\
& +2 q^{12}+\cdots,
\end{aligned}
$$

with $q=e^{2 \pi i \tau}$. The modular forms of higher weights can be constructed from the tensor products of $Y_{2}^{(1)}$. There are three linearly independent weight- 2 modular forms which can be arranged into a $T^{\prime}$ triplet,

$$
Y_{\mathbf{3}}^{(2)} \equiv\left(\begin{array}{c}
Y_{\mathbf{3}, 1}^{(2)}(\tau) \\
Y_{\mathbf{3}, 2}^{(2)}(\tau) \\
Y_{3,3}^{(2)}(\tau)
\end{array}\right)=\left(\begin{array}{c}
e^{i \pi / 6} Y_{2}^{2} \\
\sqrt{2} e^{i\urcorner \pi / 12} Y_{1} Y_{2} \\
Y_{1}^{2}
\end{array}\right)
$$

At weight 3, we have four independent modular forms which can be decomposed into two doublets transforming in the representations $\mathbf{2}$ and $\mathbf{2}^{\prime \prime}$ of $T^{\prime}$,

$$
\begin{aligned}
& Y_{\mathbf{2}}^{(3)} \equiv\left(\begin{array}{c}
Y_{\mathbf{2}, 1}^{(3)} \\
Y_{\mathbf{2}, 2}^{(3)}
\end{array}\right)=\left(\begin{array}{c}
3 e^{i \pi / 6} Y_{1} Y_{2}^{2} \\
\sqrt{2} e^{i 5 \pi / 12} Y_{1}^{3}-e^{i \pi / 6} Y_{2}^{3}
\end{array}\right), \\
& Y_{\mathbf{2}^{\prime \prime}}^{(3)} \equiv\left(\begin{array}{c}
Y_{\mathbf{2}^{\prime \prime}, 1}^{(3)} \\
Y_{\mathbf{2}^{\prime \prime}, 2}^{(3)}
\end{array}\right)=\left(\begin{array}{c}
Y_{1}^{3}+(1-i) Y_{2}^{3} \\
-3 Y_{2} Y_{1}^{2}
\end{array}\right) .
\end{aligned}
$$

The modular forms of weight 4 can be obtained from the contractions of $Y_{\mathbf{2}}^{(1)}$ and $Y_{\mathbf{2}}^{(3)}, Y_{\mathbf{2}^{\prime \prime}}^{(3)}$, and they decompose as $\mathbf{3} \oplus \mathbf{1} \oplus \mathbf{1}^{\prime}$ under $T^{\prime}$,

$$
\begin{gathered}
Y_{\mathbf{1}}^{(4)}=-4 Y_{1}^{3} Y_{2}-(1-i) Y_{2}^{4}, \\
Y_{\mathbf{3}}^{(4)}=\sqrt{2} e^{i 5 \pi / 12} Y_{1}^{4}-4 e^{i \pi / 6} Y_{1} Y_{2}^{3}, \\
Y_{\mathbf{3}, 1}^{(4)} \equiv\left(\begin{array}{c}
\sqrt{2} e^{i 7 \pi / 12} Y_{1}^{3} Y_{2}-e^{i \pi / 3} Y_{2}^{4} \\
Y_{\mathbf{3}, 2}^{(4)} \\
Y_{\mathbf{3}, 3}^{(4)}
\end{array}\right)=\left(\begin{array}{c}
1-i) Y_{1} Y_{2}^{3} \\
3 e^{i \pi / 6} Y_{1}^{2} Y_{2}^{2}
\end{array}\right) .
\end{gathered}
$$

TABLE I. Summary of modular forms of level 3 up to weight 8, where the subscript $\mathbf{r}$ denotes the transformation property under $T^{\prime}$ modular symmetry. Here $Y_{\mathbf{3} I}^{(6)}$ and $Y_{\mathbf{3} I I}^{(6)}$ stand for two weight-6 modular forms transforming in the representation 3 of $T^{\prime}$. Similar conventions are adopted for $Y_{2 I}^{(7)}, Y_{2 I I}^{(7)}$ and $Y_{3 I}^{(8)}, Y_{3 I I}^{(8)}$.

\begin{tabular}{lc}
\hline \hline Modular weight $k$ & Modular form $Y_{\mathbf{r}}^{(k)}$ \\
\hline$k=1$ & $Y_{\mathbf{2}}^{(1)}$ \\
$k=2$ & $Y_{\mathbf{3}}^{(2)}$ \\
$k=3$ & $Y_{2}^{(3)}, Y_{\mathbf{2}^{\prime \prime}}^{(3)}$ \\
$k=4$ & $Y_{\mathbf{1}}^{(4)}, Y_{\mathbf{1}^{\prime}}^{(4)}, Y_{\mathbf{3}}^{(4)}$ \\
$k=5$ & $Y_{2}^{(5)}, Y_{\mathbf{2}^{\prime}}^{(5)}, Y_{\mathbf{2}^{\prime \prime}}^{(5)}$ \\
$k=6$ & $Y_{\mathbf{1}}^{(6)}, Y_{\mathbf{3}}^{(6)}, Y_{\mathbf{3}}^{(6)}$ \\
$k=7$ & $Y_{\mathbf{2 I}}^{(7)}, Y_{\mathbf{2 I I}}^{(7)}, Y_{\mathbf{2}^{\prime}}^{(7)}, Y_{\mathbf{2}^{\prime \prime}}^{(7)}$ \\
$k=8$ & $Y_{\mathbf{1}}^{(8)}, Y_{\mathbf{1}^{\prime}}^{(8)}, Y_{\mathbf{1}^{\prime \prime}}^{(8)}, Y_{\mathbf{3}}^{(8)}, Y_{\mathbf{3}}^{(8)}$ \\
\hline \hline
\end{tabular}

The analytical expressions of modular forms of weights 5 , 6,7 , and 8 are reported in Appendix B. The modular multiplets of level 3 at different weights are summarized in Table I. It is remarkable that the odd-weight modular forms always transform in the doublet irreducible representations $\mathbf{2}, \mathbf{2}^{\prime}$, and $\mathbf{2}^{\prime \prime}$ of $T^{\prime}$, while the even-weight modular forms arrange themselves into the $T^{\prime}$ triplet 3 and singlets $\mathbf{1}, \mathbf{1}^{\prime}$, and $\mathbf{1}^{\prime \prime}$ which are identical to the representation matrices of $A_{4}$ in our working basis. This interesting structure of modular forms can help to produce texture zeros of the quark mass matrix, as we show in the following.

We formulate our models in the framework of $\mathcal{N}=1$ global supersymmetry. In modular invariant theory, it is generally assumed that the chiral supermultiplet $\Phi_{I}$ carries a modular weight $-k_{I}$ and transforms according to a certain representation $\rho_{I}$ of $\Gamma_{N}^{\prime}[11,72,73]$,

$\tau \rightarrow \gamma \tau=\frac{a \tau+b}{c \tau+d}, \quad \Phi_{I} \rightarrow(c \tau+d)^{-k_{I}} \rho_{I}(\gamma) \Phi_{I}$.

In the present paper, we choose a minimal form of the Kähler potential,

$\mathcal{K}\left(\tau, \bar{\tau}, \Phi_{I}, \bar{\Phi}_{I}\right)=-h \Lambda^{2} \log (-i \tau+i \bar{\tau})+\sum_{I} \frac{\left|\Phi_{I}\right|^{2}}{(-i \tau+i \bar{\tau})^{k_{I}}}$,

which is invariant up to a Kähler transformation under the modular transformations given in Eq. (14), and $h$ is a positive constant. It is the leading-order Kähler potential of a general matter field $\Phi_{I}$ with modular weight $-k_{I}$ in the context of string compactifications on Abelian orbifolds [74]. The Kähler potential is less constrained by modular symmetry: the operators $(-i \tau+i \bar{\tau})^{k-k_{I}}\left(\Phi_{I}^{\dagger} Y_{\mathbf{r}}^{(k) \dagger} Y_{\mathbf{r}}^{(k)} \Phi_{I}\right)_{1}$ for any integer $k$ and irreducible representation $\mathbf{r}$ cannot be avoided, and they can lead to off-diagonal elements of 
the Kähler metric and the predictions for neutrino masses and mixing parameters can be changed considerably [30]. As a consequence, in the bottom-up model construction based on modular symmetry models [11], it is necessary to better constrain the Kähler potential to enhance the predictive power. Motivated by top-down model building in string theory, the modular flavor symmetry is extended to combine with traditional flavor symmetry $[25,29,75,76]$. In this new scheme, both the superpotential and Kähler potential are strongly constrained, and the nondiagonal contributions to the Kähler metric are forbidden by all symmetries of the theory. Therefore, the extra terms depending on modular forms in the Kähler potential do not considerably alter the phenomenological predictions which have been obtained by using just the standard Kähler potential in Eq. (15) [76]. Hence, we mainly focus on the minimal canonical Kähler potential of Eq. (15) in the present work, and the nonminimal Kähler potential is discussed in Sec. VII and Appendix C. The superpotential $\mathcal{W}\left(\tau, \Phi_{I}\right)$ can be expanded in power series of the supermultiplets $\Phi_{I}$ as follows:

$$
\mathcal{W}\left(\tau, \Phi_{I}\right)=\sum_{n} Y_{I_{1} \ldots I_{n}}(\tau) \Phi_{I_{1}} \ldots \Phi_{I_{n}}
$$

where the function $Y_{I_{1} \ldots I_{n}}(\tau)$ should consist of modular forms of weight $k_{Y}$ and level $N$, and transform in the representation $\rho_{Y}$ of $\Gamma_{N}^{\prime}$,

$$
\begin{aligned}
\tau \rightarrow \gamma \tau & =\frac{a \tau+b}{c \tau+d}, \\
Y_{I_{1} \ldots I_{n}}(\tau) \rightarrow Y_{I_{1} \ldots I_{n}}(\gamma \tau) & =(c \tau+d)^{k_{Y}} \rho_{Y}(\gamma) Y_{I_{1} \ldots I_{n}}(\tau) .
\end{aligned}
$$

The requirement that $\mathcal{W}$ be invariant under the modular transformation implies that $k_{Y}$ and $\rho_{Y}$ must fulfill the following constraints:

$$
k_{Y}=k_{I_{1}}+k_{I_{1}}+\cdots+k_{I_{n}}, \quad \rho_{Y} \otimes \rho_{I_{1}} \otimes \ldots \rho_{I_{n}} \supset \mathbf{1},
$$

where 1 denotes the invariant singlet representation of $\Gamma_{N}^{\prime}$. In the following, we investigate the possible texture-zero structures of the quark mass matrix for the assignments where the three generations of right-handed quark fields either transform as a doublet and a singlet under $T^{\prime}$ modular symmetry, or are three singlets of $T^{\prime}$.

\section{QUARK MASS MATRICES FOR DOUBLET PLUS SINGLET ASSIGNMENTS OF THE RIGHT-HANDED QUARKS}

In this case, both left-handed and right-handed quark fields transform as a direct sum of one-dimensional and two-dimensional representations of the $T^{\prime}$ modular group, i.e.,

$$
\begin{aligned}
Q_{D} & \equiv\left(\begin{array}{l}
Q_{1} \\
Q_{2}
\end{array}\right) \sim \mathbf{2}^{i}, \quad Q_{3} \sim \mathbf{1}^{j}, \\
q_{D}^{c} & \equiv\left(\begin{array}{l}
q_{1}^{c} \\
q_{2}^{c}
\end{array}\right) \sim \mathbf{2}^{k}, \quad q_{3}^{c} \sim \mathbf{1}^{l},
\end{aligned}
$$

where $i, j, k, l=0,1,2$ and we have denoted $\mathbf{1} \equiv \mathbf{1}^{0}$, $\mathbf{1}^{\prime} \equiv \mathbf{1}^{1}, \mathbf{1}^{\prime \prime} \equiv \mathbf{1}^{2}$ for singlet representations and $\mathbf{2} \equiv \mathbf{2}^{0}$, $\mathbf{2}^{\prime} \equiv \mathbf{2}^{1}, \mathbf{2}^{\prime \prime} \equiv \mathbf{2}^{2}$ for the doublet representations. The notations $Q_{i}$ and $q_{i}^{c}(i=1,2,3)$ stand for the left-handed and right-handed quark fields, respectively, and $q_{i}^{c}$ can be either up-type quark fields $u_{i}^{c}$ or down-type quark fields $d_{i}^{c}$. In the following we denote the modular weights of $Q_{D}, Q_{3}$, $q_{D}^{c}$, and $q_{3}^{c}$ as $k_{Q_{D}}, k_{Q_{3}}, k_{q_{D}^{c}}$, and $k_{q_{3}^{c}}$, respectively, and the modular weights of the Higgs doublets $H_{u, d}$ are assumed to be vanishing. Thus, the most general form of the superpotential for the quark masses is given by

$$
\begin{aligned}
\mathcal{W}_{q}= & q_{D}^{c} Q_{D} H_{u / d} f_{D D}(Y)+q_{D}^{c} Q_{3} H_{u / d} f_{D 3}(Y) \\
& +q_{3}^{c} Q_{D} H_{u / d} f_{3 D}(Y)+q_{3}^{c} Q_{3} H_{u / d} f_{33}(Y),
\end{aligned}
$$

where $f_{D D}(Y), f_{D 3}(Y), f_{3 D}(Y)$, and $f_{33}(Y)$ are general functions of modular forms, and their explicit forms depend on the group indices $i, j, k, l$ and the modular weights of the quark fields. The coupling constants in front of each term are neglected in Eq. (20). The Higgs field in Eq. (20) is $H_{u}$ for up-type quark fields $u^{c}$ and $H_{d}$ for down-type quark fields $d^{c}$. Each term of Eq. (20) should be a singlet under $T^{\prime}$, and its modular weight should be zero. Moreover, from Eq. (20) we see that the quark mass matrix can be divided into four blocks as follows:

$$
M_{q}=\left(\begin{array}{ccc}
S & \vdots & C \\
\cdots & \ldots & \ldots \\
R & \vdots & T
\end{array}\right) v_{u / d},
$$

where $v_{u / d}$ is the VEV of the Higgs field $H_{u / d}$, the quark mass matrix $M_{q}$ is given in the right-left basis, and $q_{i}^{c}\left(M_{q}\right)_{i j} Q_{j}, S, C, R$, and $T$ are $2 \times 2,2 \times 1,1 \times 2$, and $1 \times 1$ submatrices, respectively. Notice that we can assign the first-generation or second-generation quark field instead of the third-generation quark field to be a singlet under $T^{\prime}$ while the remaining two generations transform as a $T^{\prime}$ doublet; the corresponding quark mass matrix can be obtained by multiplying certain permutation matrices on both sides. As a consequence, the results for the quark masses and mixing matrix are not changed. Using the Kronecker products and the CG coefficients of $T^{\prime}$ given in Appendix A, we can determine the explicit forms of $S, C$, $R$, and $T$, and each matrix element can be expressed in terms of modular forms. We are concerned with modular forms up to weight 6 in the present work; higher-weight modular forms can be discussed in a similar way, but more 
modular invariant interactions and free parameters would be involved.

\section{A. General structure of $S$}

This $2 \times 2$ submatrix is determined by the representations $\mathbf{2}^{i}$ and $\mathbf{2}^{k}$ as well as the modular weights $k_{Q_{D}}$ and $k_{q_{D}^{c}}$. Modular invariance requires that $f_{D D}(Y)$ should be a modular form in the triplet representation $\mathbf{3}$ or singlet representations $\mathbf{1}, \mathbf{1}^{\prime}, \mathbf{1}^{\prime \prime}$ of $T^{\prime}$. Hence, $S$ vanishes if $k_{q_{D}^{c}}+$ $k_{Q_{D}}$ is odd. As a consequence, the rank of the quark mass matrix would be less than three and at least one quark would be massless, which is disfavored by experimental data. For all possible assignments of $\mathbf{2}^{i}$ and $\mathbf{2}^{k}$, we can find the following possible structures for the $2 \times 2$ submatrix $S$.

(1) $\mathbf{2}^{i} \otimes \mathbf{2}^{k}=\mathbf{2} \otimes \mathbf{2}=\mathbf{2}^{\prime} \otimes \mathbf{2}^{\prime \prime}=\mathbf{3} \oplus \mathbf{1}^{\prime}$

In this case, if the summation of the modular weights $k_{Q_{D}}$ and $k_{q_{D}^{c}}$ is equal to 2, i.e., $k_{q_{D}^{c}}+k_{Q_{D}}=$ 2, $f_{D D}(Y)$ would be uniquely proportional to the weight-2 modular form $Y_{\mathbf{3}}^{(2)}=\left(Y_{\mathbf{3}, 1}^{(2)}, Y_{\mathbf{3}, 2}^{(2)}, Y_{\mathbf{3}, 3}^{(2)}\right)^{T}$ which couples to $q_{D}^{c}$ and $Q_{D}$ to form a modularinvariant singlet. From the CG coefficients of $T^{\prime}$ given in Appendix A, we can straightforwardly write the general form of $S$ as

$$
S_{1}=\left(\begin{array}{cc}
\kappa \sqrt{2} e^{\frac{i 5 \pi}{12}} Y_{\mathbf{3}, 2}^{(2)} & -\kappa Y_{\mathbf{3}, 3}^{(2)} \\
-\kappa Y_{\mathbf{3}, 3}^{(2)} & \kappa \sqrt{2} e^{\frac{i 7 \pi}{12}} Y_{\mathbf{3}, 1}^{(2)}
\end{array}\right)
$$

which is a symmetric matrix, and $\kappa$ is in general a complex Yukawa coupling parameter. In the case where $k_{q_{D}^{c}}+k_{Q_{D}}=4, f_{D D}(Y)$ would be the weight4 modular form $Y_{\mathbf{3}}^{(4)}=\left(Y_{\mathbf{3}, 1}^{(4)}, Y_{\mathbf{3}, 2}^{(4)}, Y_{\mathbf{3}, 3}^{(4)}\right)^{T}$, and the submatrix $S$ would be

$$
S_{2}=\left(\begin{array}{cc}
\kappa \sqrt{2} e^{\frac{i 5 \pi}{12}} Y_{\mathbf{3}, 2}^{(4)} & -\kappa Y_{\mathbf{3}, 3}^{(4)} \\
-\kappa Y_{\mathbf{3}, 3}^{(4)} & \kappa \sqrt{2} e^{\frac{i 7 \pi}{12}} Y_{\mathbf{3}, 1}^{(4)}
\end{array}\right)
$$

We have two independent modular forms $Y_{3 I}^{(6)}$ and $Y_{3 I I}^{(6)}$ at weight 6 , as shown in Table I. Thus, $f_{D D}(Y)$ is a linear combination of $Y_{\mathbf{3 I}}^{(6)}$ and $Y_{\mathbf{3} I I}^{(6)}$ for $k_{q_{D}^{c}}+k_{Q_{D}}=6$, and we have

$$
S_{3}=\left(\begin{array}{cc}
\sqrt{2} e^{\frac{i 5 \pi}{12}}\left(\kappa_{1} Y_{\mathbf{3} I, 2}^{(6)}+\kappa_{2} Y_{\mathbf{3} I I, 2}^{(6)}\right) & -\left(\kappa_{1} Y_{\mathbf{3} I, 3}^{(6)}+\kappa_{2} Y_{\mathbf{3} I I, 3}^{(6)}\right) \\
-\left(\kappa_{1} Y_{\mathbf{3} I, 3}^{(6)}+\kappa_{2} Y_{\mathbf{3} I I, 3}^{(6)}\right) & \sqrt{2} e^{\frac{i 7 \pi}{12}}\left(\kappa_{1} Y_{\mathbf{3} I, 1}^{(6)}+\kappa_{2} Y_{\mathbf{3} I I, 1}^{(6)}\right)
\end{array}\right),
$$

where $\kappa_{1}$ and $\kappa_{2}$ are complex free parameters.

(2) $\mathbf{2}^{i} \otimes 2^{k}=\mathbf{2} \otimes \mathbf{2}^{\prime}=\mathbf{2}^{\prime \prime} \otimes \mathbf{2}^{\prime \prime}=\mathbf{3} \oplus \mathbf{1}^{\prime \prime}$

In this case, $f_{D D}(Y)$ has to be a modular form transforming as $\mathbf{3}$ or $\mathbf{1}^{\prime}$ in order to fulfill modular invariance. Hence, $f_{D D}(Y)$ is proportional to $Y_{\mathbf{3}}^{(2)}$ for the value $k_{q_{D}^{c}}+k_{Q_{D}}=2$, and $S$ is of the following form:

$$
S_{4}=\left(\begin{array}{cc}
\kappa \sqrt{2} e^{\frac{i 5 \pi}{12}} Y_{\mathbf{3}, 1}^{(2)} & -\kappa Y_{\mathbf{3}, 2}^{(2)} \\
-\kappa Y_{\mathbf{3}, 2}^{(2)} & \kappa \sqrt{2} e^{\frac{i 7 \pi}{12}} Y_{\mathbf{3}, 3}^{(2)}
\end{array}\right)
$$

For the value of $k_{q_{D}^{c}}+k_{Q_{D}}=4$, both $Y_{\mathbf{3}}^{(4)}$ and $Y_{\mathbf{1}^{\prime}}^{(4)}$ can contribute to the quark mass terms, and the submatrix $S$ is given by

$$
S_{5}=\left(\begin{array}{cc}
\kappa_{1} \sqrt{2} e^{\frac{i 5 \pi}{12}} Y_{\mathbf{3}, 1}^{(4)} & \kappa_{2} Y_{\mathbf{1}^{\prime}}^{(4)}-\kappa_{1} Y_{\mathbf{3}, 2}^{(4)} \\
-\kappa_{2} Y_{\mathbf{1}^{\prime}}^{(4)}-\kappa_{1} Y_{\mathbf{3}, 2}^{(4)} & \kappa_{1} \sqrt{2} e^{\frac{i 7 \pi}{12}} Y_{\mathbf{3}, 3}^{(4)}
\end{array}\right)
$$

which is not a symmetric matrix because of the term $\kappa_{2}\left(q_{D}^{c} Q_{D}\right)_{\mathbf{1}^{\prime \prime}} Y_{\mathbf{1}^{\prime}}^{(4)} H_{u / d}$. Similarly, for $k_{q_{D}^{c}}+k_{Q_{D}}=6$ both $Y_{\mathbf{3}}^{(6)}$ and $Y_{\mathbf{3} I I}^{(6)}$ are relevant, and we find

$$
S_{6}=\left(\begin{array}{cc}
\sqrt{2} e^{\frac{i 5 \pi}{12}}\left(\kappa_{1} Y_{\mathbf{3} I, 1}^{(6)}+\kappa_{2} Y_{\mathbf{3} I I, 1}^{(6)}\right) & -\left(\kappa_{1} Y_{\mathbf{3} I, 2}^{(6)}+\kappa_{2} Y_{\mathbf{3} I I, 2}^{(6)}\right) \\
-\left(\kappa_{1} Y_{\mathbf{3} I, 2}^{(6)}+\kappa_{2} Y_{\mathbf{3} I I, 2}^{(6)}\right) & \sqrt{2} e^{\frac{i 7 \pi}{12}}\left(\kappa_{1} Y_{\mathbf{3} I, 3}^{(6)}+\kappa_{2} Y_{\mathbf{3} I I, 3}^{(6)}\right)
\end{array}\right)
$$

(3) $2^{i} \otimes 2^{k}=\mathbf{2} \otimes 2^{\prime \prime}=2^{\prime} \otimes 2^{\prime}=3 \oplus 1$

If the modular weights satisfy $k_{q_{D}^{c}}+k_{Q_{D}}=2$, the mass term of the first two generations of quarks is given by $\kappa\left(q_{D}^{c} Q_{D} Y_{\mathbf{3}}^{(2)}\right)_{\mathbf{1}} H_{u / d}$, which gives rise to 


$$
S_{7}=\left(\begin{array}{cc}
\kappa \sqrt{2} e^{\frac{i 5 \pi}{12}} Y_{\mathbf{3}, 3}^{(2)} & -\kappa Y_{\mathbf{3}, 1}^{(2)} \\
-\kappa Y_{\mathbf{3}, 1}^{(2)} & \kappa \sqrt{2} e^{\frac{i 7 \pi}{12}} Y_{\mathbf{3}, 2}^{(2)}
\end{array}\right)
$$

For $k_{q_{D}^{c}}+k_{Q_{D}}=4, f_{D D}(Y)$ can be the weight-4 modular forms $Y_{\mathbf{3}}^{(4)}$ and $Y_{\mathbf{1}}^{(4)}$. We can write the general form of the submatrix $S$ as follows:

$$
S_{8}=\left(\begin{array}{cc}
\kappa_{1} \sqrt{2} e^{\frac{i 5 \pi}{12}} Y_{\mathbf{3}, 3}^{(4)} & \kappa_{2} Y_{\mathbf{1}}^{(4)}-\kappa_{1} Y_{\mathbf{3}, 1}^{(4)} \\
-\kappa_{2} Y_{\mathbf{1}}^{(4)}-\kappa_{1} Y_{\mathbf{3}, 1}^{(4)} & \kappa_{1} \sqrt{2} e^{\frac{i 7 \pi}{12}} Y_{\mathbf{3}, 2}^{(4)}
\end{array}\right)
$$

For the value of $k_{q_{D}^{c}}+k_{Q_{D}}=6$, all three of the weight-6 modular forms $Y_{3 I}^{(6)}, Y_{3 I I}^{(6)}$, and $Y_{1}^{(6)}$ are relevant, and $S$ is given by

$$
S_{9}=\left(\begin{array}{cc}
\sqrt{2} e^{\frac{i 5 \pi}{12}}\left(\kappa_{1} Y_{\mathbf{3} I, 3}^{(6)}+\kappa_{2} Y_{\mathbf{3} I I, 3}^{(6)}\right) & -\left(\kappa_{1} Y_{\mathbf{3} I, 1}^{(6)}+\kappa_{2} Y_{\mathbf{3} I I, 1}^{(6)}\right)+\kappa_{3} Y_{\mathbf{1}}^{(6)} \\
-\left(\kappa_{1} Y_{\mathbf{3} I, 1}^{(6)}+\kappa_{2} Y_{\mathbf{3} I I, 1}^{(6)}\right)-\kappa_{3} Y_{\mathbf{1}}^{(6)} & \sqrt{2} e^{\frac{i 7 \pi}{12}}\left(\kappa_{1} Y_{\mathbf{3} I, 2}^{(6)}+\kappa_{2} Y_{\mathbf{3} I I, 2}^{(6)}\right)
\end{array}\right)
$$

We summarize all of the above possible forms of the submatrix $S$ for different assignments of $\mathbf{2}^{i}, \mathbf{2}^{k}$, and the modular weights $k_{Q_{D}}, k_{q_{D}^{c}}$ in Table II.

\section{B. General structures of $\boldsymbol{C}$ and $\boldsymbol{R}$}

The submatrix $C$ comprises the (13) and (23) entries of the quark mass matrix $M_{q}$, and it is determined by the modular form $f_{D 3}(Y)$. Once the assignments for $\mathbf{2}^{k}, \mathbf{1}^{j}$, and the modular weights $k_{q_{D}^{c}}$ and $k_{Q_{3}}$ are specified, we can easily find $f_{D 3}(Y)$ and the explicit form of $C$. The modular invariance requires that $q_{D}^{c}, Q_{3}$, and $f_{D 3}(Y)$ contract into a $T^{\prime}$ singlet. From the Kronecker products $\mathbf{1}^{a} \otimes \mathbf{2}^{b}=$ $2^{a+b(\bmod 3)}$ in Eq. (A2), we know that $f_{D 3}(Y)$ has to be a modular form transforming as $\mathbf{2}^{2-a-b(\bmod 3)}$. As shown in Table I, the modular weights of the doublet modular forms must be odd. Therefore, the sum $k_{q_{D}^{c}}+k_{Q_{3}}$ should be an odd integer; otherwise, the submatrix $C$ would be zero. We find that $C$ vanishes exactly if any of the following conditions are fulfilled:

$$
\begin{aligned}
& k_{q_{D}^{c}}+k_{Q_{3}}=0,2,4,6, \ldots, \\
& \text { or } \quad k_{q_{D}^{c}}+k_{Q_{3}}=1 \quad \text { with } \quad \mathbf{2}^{k} \otimes \mathbf{1}^{j}=\mathbf{2} \text { or } \mathbf{2}^{\prime}, \\
& \text { or } \quad k_{q_{D}^{c}}+k_{Q_{3}}=3 \quad \text { with } \quad \mathbf{2}^{k} \otimes \mathbf{1}^{j}=\mathbf{2}^{\prime} .
\end{aligned}
$$

\begin{tabular}{|c|c|c|}
\hline & Expressions of $S$ & Constraints \\
\hline$S_{0}$ & $\left(\begin{array}{ll}0 & 0 \\
0 & 0\end{array}\right)$ & $k_{q_{D}^{c}}+k_{Q_{D}}=0,1,3,5, \ldots$ \\
\hline$S_{1}$ & $\left(\begin{array}{cc}\kappa \sqrt{2} e^{\frac{i 5 \pi}{11}} Y_{3,2}^{(2)} & -\kappa Y_{3,3}^{(2)} \\
-\kappa Y_{3,3}^{(2)} & \kappa \sqrt{2} e^{\frac{i \pi}{12}} Y_{3,1}^{(2)}\end{array}\right)$ & $k_{q_{D}^{c}}+k_{Q_{D}}=2, k+i=0(\bmod 3)$ \\
\hline$S_{2}$ & $\left(\begin{array}{cc}\kappa \sqrt{2} e^{i 5 \pi} \frac{i 5 \pi}{11} Y_{3,2}^{(4)} & -\kappa Y_{3,3}^{(4)} \\
-\kappa Y_{3,3}^{(4)} & \kappa \sqrt{2} e^{i \frac{17 \pi}{12}} Y_{3,1}^{(4)}\end{array}\right)$ & $k_{q_{D}^{c}}+k_{Q_{D}}=4, k+i=0(\bmod 3)$ \\
\hline$S_{3}$ & 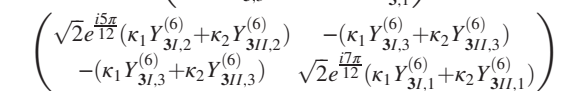 & $k_{q_{D}^{c}}+k_{Q_{D}}=6, k+i=0(\bmod 3)$ \\
\hline$S_{4}$ & $\left(\begin{array}{cc}\kappa \sqrt{2} e^{i \frac{i j \pi}{12}} Y_{3,1}^{(2)} & -\kappa Y_{3,2}^{(2)} \\
-\kappa Y_{3,2}^{(2)} & \kappa \sqrt{2} e^{\frac{i \pi n}{12}} Y_{3,3}^{(2)}\end{array}\right)$ & $k_{q_{D}^{c}}+k_{Q_{D}}=2, k+i=1(\bmod 3)$ \\
\hline$S_{5}$ & $\left(\begin{array}{cc}\kappa_{1} \sqrt{2} e^{15 \pi} \frac{15 \pi}{12} Y_{3,1}^{(4)} & \kappa_{2} Y_{1^{\prime}}^{(4)}-\kappa_{1} Y_{3,2}^{(4)} \\
-\kappa_{2} Y_{1^{\prime}}^{(4)}-\kappa_{1} Y_{3,2}^{(4)} & \kappa_{1} \sqrt{2} e^{\frac{i 7 \pi}{12}} Y_{3,3}^{(4)}\end{array}\right)$ & $k_{q_{D}^{c}}+k_{Q_{D}}=4, k+i=1(\bmod 3)$ \\
\hline$S_{6}$ & $\left(\begin{array}{cc}\sqrt{2} e^{\frac{i 5 \pi}{12}\left(\kappa_{1} Y_{3,1}^{(6)}+\kappa_{2} Y_{3 l l, 1}^{(6)}\right)} & -\left(\kappa_{1} Y_{3 l, 2}^{(6)}+\kappa_{2} Y_{3 l, 2}^{(6)}\right) \\
-\left(\kappa_{1} Y_{3 l, 2}^{(6)}+\kappa_{2} Y_{3 l l, 2}^{(6)}\right) & \sqrt{2} e^{i \frac{17}{12}\left(\kappa_{1} Y_{3 l, 3}^{(6)}+\kappa_{2} Y_{3 l l, 3}^{(6)}\right)}\end{array}\right)$ & $k_{q_{D}^{c}}+k_{Q_{D}}=6, k+i=1(\bmod 3)$ \\
\hline$S_{7}$ & $\left(\begin{array}{cc}\kappa \sqrt{2} e^{i \frac{i 5 \pi}{12}} Y_{3,3}^{(2)} & -\kappa Y_{3,1}^{(2)} \\
-\kappa Y_{3,1}^{(2)} & \kappa \sqrt{2} e^{\frac{77 \pi}{12}} Y_{3,2}^{(2)}\end{array}\right)$ & $k_{q_{D}^{c}}+k_{Q_{D}}=2, k+i=2(\bmod 3)$ \\
\hline$S_{8}$ & 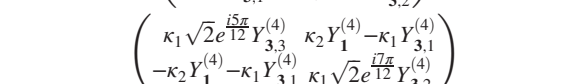 & $k_{q_{D}^{c}}+k_{Q_{D}}=4, k+i=2(\bmod 3)$ \\
\hline$S_{9}$ & 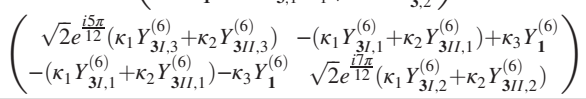 & $k_{q_{D}^{c}}+k_{Q_{D}}=6, k+i=2(\bmod 3)$ \\
\hline
\end{tabular}

In the case that $C$ is nonvanishing, it can take the following nontrivial forms.

TABLE II. The possible structures of the submatrix $S$, where the left-handed quark doublet $Q_{D}$ and the righthanded quark field $q_{D}^{c}$ are assigned to transform as $\mathbf{2}^{i}$ and $\mathbf{2}^{k}$, respectively, under $T^{\prime}$ modular symmetry, and their modular weights are denoted as $k_{Q_{D}}$ and $k_{q_{D}^{c}}$, respectively. 
(1) $\mathbf{2}^{k} \otimes \mathbf{1}^{j}=\mathbf{2}$ for $k+j=0(\bmod 3)$

In this case, $f_{D 3}(Y)$ should be a modular form transforming as $\mathbf{2}^{\prime \prime}$ under $T^{\prime}$. From Table I, we see that modular forms in the representation $\mathbf{2}^{\prime \prime}$ appear at weight 3 and weight 5 . For $k_{q_{D}^{c}}+k_{Q_{3}}=3, f_{D 3}(Y)$ is proportional to $Y_{\mathbf{2}^{\prime \prime}}^{(3)} \equiv\left(Y_{\mathbf{2}^{\prime \prime}, 1}^{(3)}, Y_{\mathbf{2}^{\prime \prime}, 2}^{(3)}\right)^{T}$, and the corresponding structure of $C$ is given by

$$
C_{1}=\left(\kappa Y_{\mathbf{2}^{\prime \prime}, 2}^{(3)},-\kappa Y_{\mathbf{2}^{\prime \prime}, 1}^{(3)}\right)^{T} .
$$

For $k_{q_{D}^{c}}+k_{Q_{3}}=5$, we can easily write the submatrix $C$ as

$$
C_{2}=\left(\kappa Y_{\mathbf{2}^{\prime \prime}, 2}^{(5)},-\kappa Y_{\mathbf{2}^{\prime \prime}, 1}^{(5)}\right)^{T} .
$$

(2) $\mathbf{2}^{k} \otimes \mathbf{1}^{j}=\mathbf{2}^{\prime}$ for $k+j=1(\bmod 3)$

The modular form $f_{D 3}(Y)$ should transform as $\mathbf{2}^{\prime}$ for this assignment. As shown in Table I, the lowestweight modular form in the doublet representation $\mathbf{2}^{\prime}$ is $Y_{\mathbf{2}^{\prime}}^{(5)}=\left(Y_{\mathbf{2}^{\prime}, 1}^{(5)}, Y_{\mathbf{2}^{\prime}, 2}^{(5)}\right)^{T}$. Accordingly, the relevant quark mass term is $\left(q_{D}^{c} Q_{3} Y_{\mathbf{2}^{\prime}}^{(5)}\right)_{\mathbf{1}} H_{u / d}$ for $k_{q_{D}^{c}}+k_{Q_{3}}=5$, and the block $C$ has the following form:

$$
C_{3}=\left(\kappa Y_{\mathbf{2}^{\prime}, 2}^{(5)},-\kappa Y_{\mathbf{2}^{\prime}, 1}^{(5)}\right)^{T} .
$$

(3) $\mathbf{2}^{k} \otimes \mathbf{1}^{j}=\mathbf{2}^{\prime \prime}$ for $k+j=2(\bmod 3)$

A modular form transforming in the doublet representation 2 of $T^{\prime}$ is necessary in order to form an invariant singlet in this case. There are three modular forms $Y_{\mathbf{2}}^{(1)}=\left(Y_{\mathbf{2}, 1}^{(1)}, Y_{\mathbf{2}, 2}^{(1)}\right)^{T}, Y_{\mathbf{2}}^{(3)}=\left(Y_{\mathbf{2}, 1}^{(3)}, Y_{\mathbf{2}, 2}^{(3)}\right)^{T}$, and $Y_{\mathbf{2}}^{(5)}=\left(Y_{\mathbf{2}, 1}^{(5)}, Y_{\mathbf{2}, 2}^{(5)}\right)^{T}$ transforming as $\mathbf{2}$. For the value $k_{q_{D}^{c}}+k_{Q_{3}}=1, Y_{\mathbf{2}}^{(1)}$ is involved, and we find that the submatrix $C$ is

$$
C_{4}=\left(\kappa Y_{\mathbf{2}, 2}^{(1)},-\kappa Y_{\mathbf{2}, 1}^{(1)}\right)^{T}
$$

For $k_{q_{D}^{c}}+k_{Q_{3}}=3$, the modular form $f_{D 3}(Y)$ is $Y_{2}^{(3)}$, and $C$ takes the form

$$
C_{5}=\left(\kappa Y_{\mathbf{2}, 2}^{(3)},-\kappa Y_{\mathbf{2}, 1}^{(3)}\right)^{T}
$$

Similarly, for $k_{q_{D}^{c}}+k_{Q_{3}}=5$, the submatrix $C$ reads as

$$
C_{6}=\left(\kappa Y_{\mathbf{2}, 2}^{(5)},-\kappa Y_{2,1}^{(5)}\right)^{T}
$$

In exactly the same fashion, we can fix the possible structures of the submatrix $R$, and the results are summarized in Table III.

\section{General structure of $\boldsymbol{T}$}

$T$ is the (33) entry of the quark mass matrix, and it is nonzero if any of the following three conditions are satisfied:

$$
\begin{aligned}
k_{q_{3}^{c}}+k_{Q_{3}} & =0, \quad \text { with } \quad \mathbf{1}^{l} \otimes \mathbf{1}^{j}=\mathbf{1}, \\
\text { or } \quad k_{q_{3}^{c}}+k_{Q_{3}}=4, & \text { with } \quad \mathbf{1}^{l} \otimes \mathbf{1}^{j}=\mathbf{1} \quad \text { or } \quad \mathbf{1}^{\prime \prime}, \\
\text { or } \quad k_{q_{3}^{c}}+k_{Q_{3}}=6, & \text { with } \quad \mathbf{1}^{l} \otimes \mathbf{1}^{j}=\mathbf{1} .
\end{aligned}
$$

\begin{tabular}{|c|c|c|c|c|c|}
\hline & Expressions of $C$ & Constraints & & Expressions of $R$ & Constraints \\
\hline$C_{0}$ & $\left(\kappa Y_{7^{\prime \prime 2},}^{(3)},-\kappa Y_{7^{\prime \prime} 1}^{(3)}\right)^{T}$ & 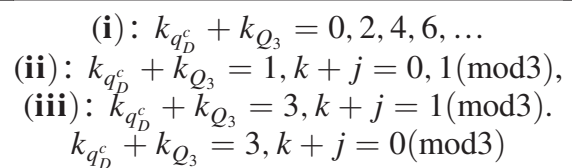 & $R_{0}$ & $\left(\kappa Y_{2^{\prime \prime} \Omega}^{(3)},-\kappa Y_{2^{\prime \prime} 1}^{(3)}\right)$ & $\begin{array}{c}\text { (i): } k_{q_{3}^{c}}+k_{Q_{D}}=0,2,4,6, \ldots \\
\text { (ii): } k_{q_{3}^{c}}+k_{Q_{D}}=1, l+i=0,1(\bmod 3), \\
\text { (iii) }: k_{q_{3}^{c}}+k_{Q_{D}}=3, l+i=1(\bmod 3) . \\
k_{q_{3}^{c}}+k_{Q_{D}}=3, l+i=0(\bmod 3)\end{array}$ \\
\hline$C_{2}$ & $\left(\kappa Y_{2^{\prime \prime 2} 2}^{(5)},-\kappa Y_{2^{\prime \prime} 1}^{(5)}\right)^{T}$ & $k_{q_{D}^{c}}+k_{Q_{3}}=5, k+j=0(\bmod 3)$ & $R_{2}$ & $\left(\kappa Y_{2^{\prime \prime} 2}^{(5)},-\kappa Y_{2^{\prime \prime} 1}^{(5)}\right)$ & $k_{q_{3}^{c}}+k_{Q_{D}}=5, l+i=0(\bmod 3)$ \\
\hline$C_{3}$ & $\left(\kappa Y_{2^{\prime},}^{(5)},-\kappa Y_{2^{\prime} 1}^{(5)}\right)^{T}$ & $k_{q_{D}^{c}}+k_{Q_{3}}=5, k+j=1(\bmod 3)$ & $R_{3}$ & $\left(\kappa Y_{2^{\prime}}^{(5)},-\kappa Y_{2^{\prime} 1}^{(5)}\right)$ & $k_{q_{3}^{c}}+k_{Q_{D}}=5, l+i=1(\bmod 3)$ \\
\hline$C_{4}$ & $\left(\kappa Y_{2,2}^{(1)},-\kappa Y_{2,1}^{(1)}\right)^{T}$ & $k_{q_{D}^{c}}+k_{Q_{3}}=1, k+j=2(\bmod 3)$ & $R_{4}$ & $\left(\kappa Y_{2,2}^{(1)},-\kappa Y_{2,1}^{(1)}\right)$ & $k_{q_{3}^{c}}+k_{Q_{D}}=1, l+i=2(\bmod 3)$ \\
\hline$C_{5}$ & $\left(\kappa Y_{2,}^{(3)},-\kappa Y_{21}^{(3)}\right)^{T}$ & $k_{q_{D}^{c}}+k_{Q_{3}}=3, k+j=2(\bmod 3)$ & $R_{5}$ & $\left(\kappa Y_{2,}^{(3)},-\kappa Y_{, 1}^{(3)}\right)$ & $k_{q_{3}^{c}}+k_{Q_{D}}=3, l+i=2(\bmod 3)$ \\
\hline$C_{6}$ & $\left(\kappa Y_{2,2}^{(5)},-\kappa Y_{2,1}^{(5)}\right)^{T}$ & $k_{q_{D}^{c}}+k_{Q_{3}}=5, k+j=2(\bmod 3)$ & $R_{6}$ & $\left(\kappa Y_{2,2}^{(5)},-\kappa Y_{2,1}^{(5)}\right)$ & $k_{q_{3}^{c}}+k_{Q_{D}}=5, l+i=2(\bmod 3)$ \\
\hline
\end{tabular}

Considering the free coupling constant associated with the term $q_{3}^{c} Q_{3} H_{u / d} f_{33}(Y)$, the above three cases essentially give the same prediction for $T$. Moreover, the element $T$ is vanishes exactly for

TABLE III. The structures of the submatrices $C$ and $R$ for different possible values of modular weights and assignments of the quark fields $q_{D}^{c}, q_{3}^{c}, Q_{D}$, and $Q_{3}$ under the finite modular group $T^{\prime}$. 


$$
\begin{aligned}
k_{q_{3}^{c}}+k_{Q_{3}} & =1,2,3,5, \\
\text { or } \quad k_{q_{3}^{c}}+k_{Q_{3}} & =4, \quad \text { with } \quad \mathbf{1}^{l} \otimes \mathbf{1}^{j}=\mathbf{1}^{\prime}, \\
\text { or } \quad k_{q_{3}^{c}}+k_{Q_{3}} & =0,6, \quad \text { with } \quad \mathbf{1}^{l} \otimes \mathbf{1}^{j}=\mathbf{1}^{\prime} \quad \text { or } \quad \mathbf{1}^{\prime \prime},
\end{aligned}
$$

up to weight-6 modular forms.

\section{Possible structures of the quark mass matrix}

Combining the possible forms of the submatrices $S, C$, $R$, and $T$ summarized in Tables II and III, we can straightforwardly obtain the quark mass matrix. It is well established that no quark is massless, and therefore we only consider quark mass matrices with nonvanishing determinants in this paper. As a result, we find that the quark mass matrix can take the following four possible forms with texture zeros:

$\operatorname{Case} \mathcal{A}:\left(\begin{array}{lll}\times & \times & 0 \\ \times & \times & 0 \\ 0 & 0 & \times\end{array}\right), \quad \operatorname{Case} \mathcal{B}:\left(\begin{array}{lll}\times & \times & \times \\ \times & \times & \times \\ 0 & 0 & \times\end{array}\right)$,
$\operatorname{Case} \mathcal{C}:\left(\begin{array}{lll}\times & \times & 0 \\ \times & \times & 0 \\ \times & \times & \times\end{array}\right), \quad \operatorname{Case} \mathcal{D}:\left(\begin{array}{lll}\times & \times & \times \\ \times & \times & \times \\ \times & \times & 0\end{array}\right)$,

where a cross denotes a nonvanishing entry. It is remarkable that the nonvanishing elements are correlated with each other in the present approach.

\section{QUARK MASS MATRICES FOR SINGLET ASSIGNMENTS OF THE RIGHT-HANDED QUARKS}

In this section, we consider another case in which the three generations of left-handed quarks are assigned to a direct sum of a doublet and singlet of $T^{\prime}$, while the three generations of right-handed quarks are assumed to transform as one-dimensional representations of the $T^{\prime}$ modular group, i.e.,

$$
Q_{D} \equiv\left(\begin{array}{c}
Q_{1} \\
Q_{2}
\end{array}\right) \sim \mathbf{2}^{i}, \quad Q_{3} \sim \mathbf{1}^{j}, \quad q_{a}^{c} \sim \mathbf{1}^{l_{a}}
$$

with $a=1,2,3$,

where $i, j, l_{1,2,3}=0,1,2$ with $\mathbf{1} \equiv \mathbf{1}^{0}, \mathbf{1}^{\prime} \equiv \mathbf{1}^{1}, \mathbf{1}^{\prime \prime} \equiv \mathbf{1}^{2}$ for singlet representations and $\mathbf{2} \equiv \mathbf{2}^{0}, \mathbf{2}^{\prime} \equiv \mathbf{2}^{1}, \mathbf{2}^{\prime \prime} \equiv \mathbf{2}^{2}$ for doublet representations. Thus, the most general superpotential for the quark masses is given by

$\mathcal{W}_{q}=\sum_{a=1}^{3} q_{a}^{c} Q_{D} H_{u / d} f_{a D}(Y)+q_{a}^{c} Q_{3} H_{u / d} f_{a 3}(Y)$, where we have suppressed all coupling constants. As a consequence, we can divide the quark mass matrix $M_{q}$ into six parts as follows:

$$
M_{q}=\left(\begin{array}{ll}
R_{1}^{\prime} & C_{1}^{\prime} \\
R_{2}^{\prime} & C_{2}^{\prime} \\
R_{3}^{\prime} & C_{3}^{\prime}
\end{array}\right),
$$

where $R_{1,2,3}^{\prime}$ and $C_{1,2,3}^{\prime}$ are $1 \times 2$ and $1 \times 1$ submatrices, respectively, and they are determined by the modular forms $f_{a D}(Y)$ and $f_{a 3}(Y)$. We will not explicitly discuss the case where the left-handed quarks transform as three onedimensional representations of $T^{\prime}$ with the right-handed quark fields assigned to a singlet and a doublet under $T^{\prime}$, since we only need to transpose the mass matrix in Eq. (43) to switch the transformation properties of left-handed and right-handed quarks.

\section{A. General structures of $\boldsymbol{R}_{a}^{\prime}$ and $\boldsymbol{C}_{a}^{\prime}$}

It is easy to see that modular invariance requires the modular form $f_{a D}(Y)$ to be in the doublet representations $2^{2-l_{a}-i(\bmod 3)}$ of $T^{\prime}$, and its modular weight should be $k_{q_{a}^{c}}+k_{Q_{D}}$. The submatrix $R_{a}^{\prime}$ vanishes exactly if any of the following relations are satisfied:

$$
\begin{aligned}
k_{q_{a}^{c}}+k_{Q_{D}} & =0,2,4, \ldots, \\
\text { or } \quad k_{q_{a}^{c}}+k_{Q_{D}} & =1, i+l_{a}=1,2 \quad(\bmod 3), \\
\text { or } \quad k_{q_{a}^{c}}+k_{Q_{D}} & =3, i+l_{a}=1 \quad(\bmod 3) .
\end{aligned}
$$

Depending on the assignments $\mathbf{2}^{i}, \mathbf{1}^{l_{a}}$ and the modular weights $k_{q_{a}^{c}}, k_{Q_{D}}, R_{a}^{\prime}$ can take the following nontrivial forms.

(1) $\mathbf{2}^{i} \otimes \mathbf{1}^{l_{a}}=\mathbf{2}$ with $i+l_{a}=0(\bmod 3)$

In this case, $f_{a D}(Y)$ should be in the representation $\mathbf{2}^{\prime \prime}$, and it can be either $Y_{\mathbf{2}^{\prime \prime}}^{(3)}$ or $Y_{\mathbf{2}^{\prime \prime}}^{(5)}$ up to weight 6 . For $k_{q_{a}^{c}}+k_{Q_{D}}=3$, we can write the submatrix $R_{a}^{\prime}$ as

$$
R_{a, 1}^{\prime}=\left(\kappa Y_{\mathbf{2}^{\prime \prime}, 2}^{(3)},-\kappa Y_{\mathbf{2}^{\prime \prime}, 1}^{(3)}\right)
$$

For $k_{q_{a}^{c}}+k_{Q_{D}}=5, R_{a}^{\prime}$ is of the form

$$
R_{a, 2}^{\prime}=\left(\kappa Y_{\mathbf{2}^{\prime \prime}, 2}^{(5)},-\kappa Y_{\mathbf{2}^{\prime \prime}, 1}^{(5)}\right)
$$

(2) $\mathbf{2}^{i} \otimes \mathbf{1}^{l_{a}}=\mathbf{2}^{\prime}$ with $i+l_{a}=1(\bmod 3)$

If we only consider the modular forms with modular weight less than 7 , then $f_{a D}(Y)$ is proportional to $Y_{2^{\prime}}^{(5)}$, and $R_{a}^{\prime}$ takes the following form:

$$
R_{a, 3}^{\prime}=\left(\kappa Y_{\mathbf{2}^{\prime}, 2}^{(5)},-\kappa Y_{\mathbf{2}^{\prime}, 1}^{(5)}\right) .
$$


(3) $\mathbf{2}^{i} \otimes \mathbf{1}^{l_{a}}=\mathbf{2}^{\prime \prime}$ with $i+l_{a}=2(\bmod 3)$

The modular invariance requires that $f_{a D}(Y)$ should transform as the doublet representation 2 of $T^{\prime}$. For $k_{q_{a}^{c}}+k_{Q_{D}}=1$, the quark mass Yukawa term is $\kappa q_{a}^{c} Q_{D} Y_{\mathbf{2}}^{(1)} H_{u / d}$ such that the submatrix $R_{a}^{\prime}$ is given by

$$
R_{a, 4}^{\prime}=\left(\kappa Y_{\mathbf{2}, 2}^{(1)},-\kappa Y_{\mathbf{2}, 1}^{(1)}\right)
$$

For $k_{q_{a}^{c}}+k_{Q_{D}}=3$, we can write $R_{a}^{\prime}$ as

$$
R_{a, 5}^{\prime}=\left(\kappa Y_{\mathbf{2}, 2}^{(3)},-\kappa Y_{\mathbf{2}, 1}^{(3)}\right)
$$

For $k_{q_{a}^{c}}+k_{Q_{D}}=5$, the weight-5 modular form $Y_{\mathbf{2}}^{(5)}=\left(Y_{\mathbf{2}, 1}^{(5)}, Y_{\mathbf{2}, 2}^{(5)}\right)^{T}$ is involved, and the submatrix $R_{a}^{\prime}$ is

$$
R_{a, 6}^{\prime}=\left(\kappa Y_{\mathbf{2}, 2}^{(5)},-\kappa Y_{2,1}^{(5)}\right)
$$

All of the above possible forms of $R_{a}^{\prime}$ are summarized in Table IV.

Since both $q_{a}^{c}$ and $Q_{3}$ are assigned to singlet representations $\mathbf{1}^{l_{a}}$ and $\mathbf{1}^{j}$, respectively, the modular form $f_{a 3}(Y)$ in the last term of Eq. (42) should transform as singlet $\mathbf{1}^{3-l_{a}-j(\bmod 3)}$ under the $T^{\prime}$ modular group. Analogous to previous cases, we find that the element $C_{a}^{\prime}$ is nonzero for

$$
\begin{aligned}
k_{q_{a}^{c}}+k_{Q_{3}} & =0, j+l_{a}=0 \quad(\bmod 3), \\
\text { or } \quad k_{q_{a}^{c}}+k_{Q_{3}} & =4, j+l_{a}=0,2 \quad(\bmod 3), \\
\text { or } \quad k_{q_{a}^{c}}+k_{Q_{3}} & =6, j+l_{a}=0 \quad(\bmod 3) .
\end{aligned}
$$

Otherwise, $C_{a}^{\prime}$ vanishes exactly, in particular when the summation of modular weights $k_{q_{a}^{c}}+k_{Q_{3}}$ is an odd integer.

TABLE IV. The structures of the submatrix $R_{a}^{\prime}$ for different possible values of modular weights and the assignments of the quark fields $q_{a}^{c}, Q_{D}$ under the finite modular group $T^{\prime}$.

\begin{tabular}{lcc}
\hline \hline \multicolumn{2}{c}{ Expressions of $R_{a}^{\prime}$} & Constraints \\
\hline$R_{a, 0}^{\prime}$ & $(0,0)$ & $(\mathbf{i}): k_{q_{a}^{c}}+k_{Q_{D}}=0,2,4,6, \ldots$ \\
& $\begin{array}{c}\text { (ii) }): k_{q_{a}^{c}}+k_{Q_{D}}=1, i+l_{a}=0,1(\bmod 3) \\
(\mathbf{i i i}): k_{q_{a}^{c}}+k_{Q_{D}}=3, i+l_{a}=1(\bmod 3)\end{array}$ \\
$R_{a, 1}^{\prime} \quad\left(\kappa Y_{2^{\prime \prime}, 2}^{(3)},-\kappa Y_{2^{\prime \prime}, 1}^{(3)}\right)$ & $k_{q_{a}^{c}}+k_{Q_{D}}=3, i+l_{a}=0(\bmod 3)$ \\
$R_{a, 2}^{\prime}$ & $\left(\kappa Y_{2^{\prime \prime}, 2}^{(5)},-\kappa Y_{\mathbf{2}^{\prime \prime}, 1}^{(5)}\right)$ & $k_{q_{a}^{c}}+k_{Q_{D}}=5, i+l_{a}=0(\bmod 3)$ \\
$R_{a, 3}^{\prime} \quad\left(\kappa Y_{2^{\prime}, 2}^{(5)},-\kappa Y_{2_{1}, 1}^{(5)}\right)$ & $k_{q_{a}^{c}}+k_{Q_{D}}=5, i+l_{a}=1(\bmod 3)$ \\
$R_{a, 4}^{\prime} \quad\left(\kappa Y_{2,2}^{(1)},-\kappa Y_{2,1}^{(1)}\right)$ & $k_{q_{a}^{c}}+k_{Q_{D}}=1, i+l_{a}=2(\bmod 3)$ \\
$R_{a, 5}^{\prime} \quad\left(\kappa Y_{2,2}^{(3)},-\kappa Y_{2,1}^{(3)}\right)$ & $k_{q_{a}^{c}}+k_{Q_{D}}=3, i+l_{a}=2(\bmod 3)$ \\
$R_{a, 6}^{\prime} \quad\left(\kappa Y_{2,2}^{(5)},-\kappa Y_{2,1}^{(5)}\right)$ & $k_{q_{a}^{c}}+k_{Q_{D}}=5, i+l_{a}=2(\bmod 3)$ \\
\hline \hline
\end{tabular}

\section{B. Possible structures of the quark mass matrix}

Given the possible forms of $R_{a}^{\prime}$ listed in Table IV, we find that the quark mass matrix can take the following five possible forms with texture zeros:

$$
\begin{aligned}
& \operatorname{Case} \mathcal{A}:\left(\begin{array}{ccc}
\times & \times & 0 \\
\times & \times & 0 \\
0 & 0 & \times
\end{array}\right), \quad \operatorname{Case} \mathcal{B}:\left(\begin{array}{ccc}
\times & \times & \times \\
\times & \times & \times \\
0 & 0 & \times
\end{array}\right), \\
& \operatorname{Case} \mathcal{C}:\left(\begin{array}{ccc}
\times & \times & 0 \\
\times & \times & 0 \\
\times & \times & \times
\end{array}\right), \quad \operatorname{Case} \mathcal{D}:\left(\begin{array}{ccc}
\times & \times & \times \\
\times & \times & \times \\
\times & \times & 0
\end{array}\right), \\
& \text { Case } \mathcal{E}:\left(\begin{array}{ccc}
\times & \times & 0 \\
\times & \times & \times \\
0 & 0 & \times
\end{array}\right),
\end{aligned}
$$

up to row and column permutations. The symbol " $\times$ " denotes a nonzero matrix element, and we have neglected the quark mass matrices with zero determinants in Eq. (52). If we exchange the assignments for the left-handed and right-handed quark fields in Eq. (41), another new texture for the quark mass matrix can be obtained:

$$
\text { Case } \mathcal{F}:\left(\begin{array}{ccc}
\times & \times & 0 \\
\times & \times & 0 \\
0 & \times & \times
\end{array}\right) .
$$

\section{PHENOMENOLOGICALLY VIABLE MODELS FOR QUARK MASSES AND CKM MIXING}

As shown in Secs. III and IV, both up-quark and downquark mass matrices can take six possible textures with zero elements-Case $\mathcal{A}$, Case $\mathcal{B}$, Case $\mathcal{C}$, Case $\mathcal{D}$, Case $\mathcal{E}$, and Case $\mathcal{F}$ given in Eqs. (40), (52), and (53) - if we properly assign the modular weights of the quark fields and their transformation properties under $T^{\prime}$. Combining the upquark sector with the down-quark sector, we can obtain the possible up-quark and down-quark mass matrices predicted by $T^{\prime}$ modular symmetry. We find that many cases can accommodate the experimental data on quark masses and the CKM mixing matrix, and the resulting predictions for quark mass matrices can be classified according to the number of zero elements and involved free parameters. In order to show concrete examples, we present five interesting models for quarks in the following.

Model I: Seven zero elements and ten free parameters

The classification of the quark fields under the standard model gauge symmetry and $T^{\prime}$ modular symmetry are listed in Table V. The quark $S U(2)$ doublets are assigned to a doublet and singlet $Q_{D} \sim 2$, $Q_{3} \sim \mathbf{1}^{\prime}$, the up-type quark $S U(2)$ singlets $u^{c}, c^{c}$, and $t^{c}$ are assigned to $\mathbf{1}^{\prime \prime}, \mathbf{1}$, and $\mathbf{1}^{\prime \prime}$, respectively, and the right-handed down-type quarks are assumed to 
TABLE V. The transformation properties of the quark fields under the standard model gauge group $S U(2)_{L} \times U(1)_{Y}$ and under $T^{\prime}$ modular symmetry for different models, where $-k_{I}$ refers to the modular weights. The two Higgs doublets $H_{u, d}$ are invariant under $T^{\prime}$ and their modular weights are assumed to be vanishing.

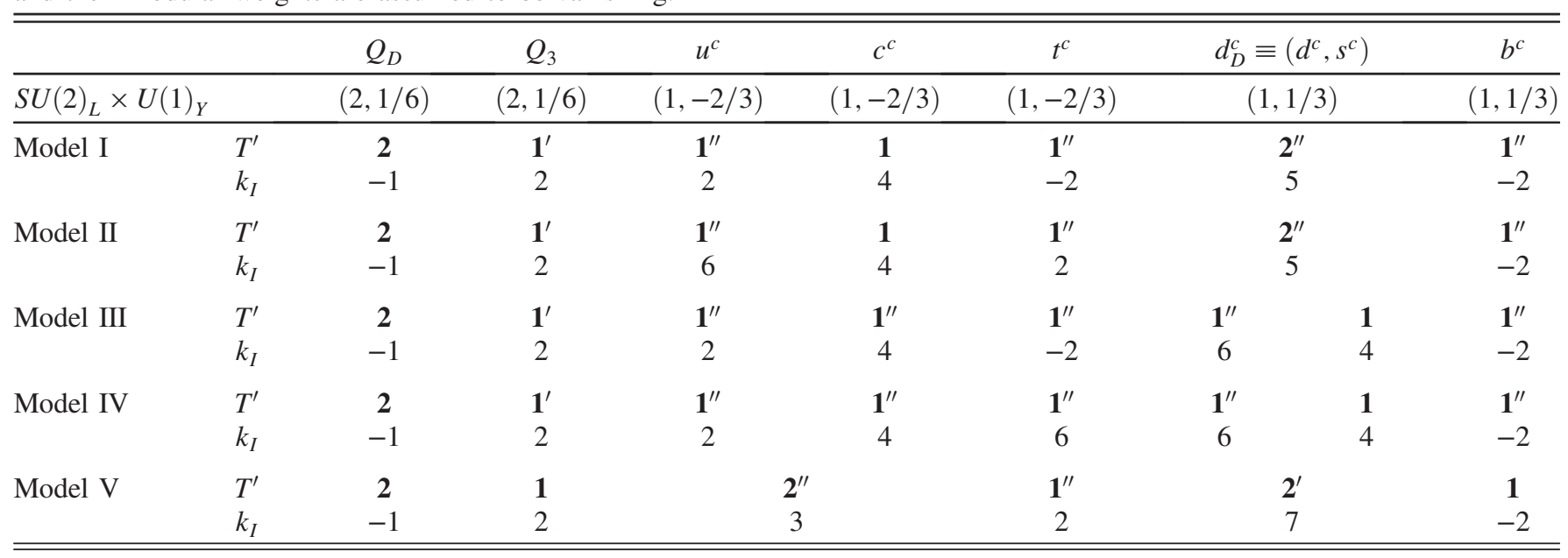

transform as a doublet and singlet $d_{D}^{c} \equiv\left(d^{c}, s^{c}\right) \sim \mathbf{2}^{\prime \prime}$, $b^{c} \sim \mathbf{1}^{\prime \prime}$. Then, we can write the modular-invariant superpotentials for the up and down sectors as follows:

$$
\begin{aligned}
\mathcal{W}_{u}= & y_{1}^{u} u^{c} Q_{D} Y_{\mathbf{2}}^{(1)} H_{u}+y_{2}^{u} u^{c} Q_{3} Y_{\mathbf{1}}^{(4)} H_{u} \\
& +y_{3}^{u} c^{c} Q_{D} Y_{\mathbf{2}^{\prime \prime}}^{(3)} H_{u}+y_{4}^{u} t^{c} Q_{3} H_{u}, \\
\mathcal{W}_{d}= & y_{1}^{d} d_{D}^{c} Q_{D} Y_{\mathbf{3}}^{(4)} H_{d}+y_{2}^{d} d_{D}^{c} Q_{D} Y_{\mathbf{1}}^{(4)} H_{d} \\
& +y_{3}^{d} b^{c} Q_{3} H_{d},
\end{aligned}
$$

where the coupling constants $y_{1,2,3,4}^{u}$ and $y_{1,3}^{d}$ can be taken to be real and positive by rephasing the quark fields without loss of generality, while the phase of $y_{2}^{d}$ cannot be removed. Applying the decomposition rules of the $T^{\prime}$ tensor products in Appendix A, we find that the quark mass matrices are given by

$$
\begin{aligned}
M_{u} & =\left(\begin{array}{ccc}
y_{1}^{u} Y_{\mathbf{2}, 2}^{(1)} & -y_{1}^{u} Y_{\mathbf{2}, 1}^{(1)} & y_{2}^{u} Y_{\mathbf{1}}^{(4)} \\
y_{3}^{u} Y_{\mathbf{2}^{\prime \prime}, 2}^{(3)} & -y_{3}^{u} Y_{\mathbf{2}^{\prime \prime}, 1}^{(3)} & 0 \\
0 & 0 & y_{4}^{u}
\end{array}\right) v_{u}, \\
M_{d} & =\left(\begin{array}{ccc}
-\sqrt{2} e^{\frac{5 i \pi}{12}} y_{1}^{d} Y_{\mathbf{3}, 3}^{(4)} & y_{1}^{d} Y_{\mathbf{3}, 1}^{(4)}-y_{2}^{d} Y_{\mathbf{1}}^{(4)} & 0 \\
y_{1}^{d} Y_{\mathbf{3}, 1}^{(4)}+y_{2}^{d} Y_{\mathbf{1}}^{(4)} & -\sqrt{2} e^{\frac{7 i \pi}{12}} y_{1}^{d} Y_{\mathbf{3}, 2}^{(4)} & 0 \\
0 & 0 & 0
\end{array}\right) v_{d}^{d} .
\end{aligned}
$$

We see that there are a total of seven zero entries in $M_{u}$ and $M_{d}$, and the down-quark mass matrix $M_{d}$ is block diagonal. Apart form the dependence of $M_{u}$ and $M_{d}$ on the VEV of the complex modulus $\tau$, we have six real input parameters $y_{1,2,3,4}^{u}, y_{1,3}^{d}$ and one complex parameter $y_{2}^{d}$ to describe the quark masses, mixing angles, and phases.
Model II: Six zero elements and ten free parameters

The transformation rules of the quarks under the $T^{\prime}$ modular symmetry are identical to those of Model I, while the assignments of the modular weights are different, as shown in Table V. The superpotential for the quark Yukawa interactions is given by

$$
\begin{aligned}
\mathcal{W}_{u}= & y_{1}^{u} u^{c} Q_{D} Y_{\mathbf{2}}^{(5)} H_{u}+y_{2}^{u} c^{c} Q_{D} Y_{\mathbf{2}^{\prime \prime}}^{(3)} H_{u} \\
& +y_{3}^{u} t^{c} Q_{D} Y_{\mathbf{2}}^{(1)} H_{u}+y_{4}^{u} t^{c} Q_{3} Y_{\mathbf{1}}^{(4)} H_{u}, \\
\mathcal{W}_{d}= & y_{1}^{d} d_{D}^{c} Q_{D} Y_{\mathbf{3}}^{(4)} H_{d}+y_{2}^{d} d_{D}^{c} Q_{D} Y_{\mathbf{1}}^{(4)} H_{d} \\
& +y_{3}^{d} b^{c} Q_{3} H_{d},
\end{aligned}
$$

where the phases of the couplings $y_{1,2,3,4}^{u}$ and $y_{1,3}^{d}$ can be absorbed into the quark fields, while the phase of $y_{2}^{d}$ cannot be eliminated by field redefinition. The superpotential in Eq. (56) leads to the following up- anddown quark mass matrices:

$$
\begin{aligned}
M_{u} & =\left(\begin{array}{ccc}
y_{1}^{u} Y_{\mathbf{2}, 2}^{(5)} & -y_{1}^{u} Y_{\mathbf{2}, 1}^{(5)} & 0 \\
y_{2}^{u} Y_{\mathbf{2}^{\prime \prime}, 2}^{(3)} & -y_{2}^{u} Y_{\mathbf{2}^{\prime \prime}, 1}^{(3)} & 0 \\
y_{3}^{u} Y_{\mathbf{2}, 2}^{(1)} & -y_{3}^{u} Y_{\mathbf{2}, 1}^{(1)} & y_{4}^{u} Y_{\mathbf{1}}^{(4)}
\end{array}\right) v_{u}, \\
M_{d} & =\left(\begin{array}{ccc}
-\sqrt{2} e^{\frac{5 i \pi}{12}} y_{1}^{d} Y_{\mathbf{3}, 3}^{(4)} & y_{1}^{d} Y_{\mathbf{3}, 1}^{(4)}-y_{2}^{d} Y_{\mathbf{1}}^{(4)} & 0 \\
y_{1}^{d} Y_{\mathbf{3}, 1}^{(4)}+y_{2}^{d} Y_{\mathbf{1}}^{(4)} & -\sqrt{2} e^{\frac{7 i \pi}{12}} y_{1}^{d} Y_{\mathbf{3}, 2}^{(4)} & 0 \\
0 & 0 & y_{3}^{d}
\end{array}\right) v_{d},
\end{aligned}
$$

which depend on ten real input parameters including the real and imaginary parts of the modulus $\tau$.

Model III: Six zero elements and 11 free parameters

In this model, we assign the three generations of left-handed quark fields to the doublet 2 and singlet $\mathbf{1}^{\prime}$, the right-handed up-type quarks $u^{c}, c^{c}$, and $t^{c}$ all 
transform as $\mathbf{1}^{\prime \prime}$, and the right-handed down-type quarks $d^{c}, s^{c}$, and $b^{c}$ transform as $\mathbf{1}^{\prime \prime}, \mathbf{1}$, and $\mathbf{1}^{\prime \prime}$, respectively. The quark masses are described by

$$
\begin{aligned}
\mathcal{W}_{u}= & y_{1}^{u} u^{c} Q_{D} Y_{2}^{(1)} H_{u}+y_{2}^{u} u^{c} Q_{3} Y_{1}^{(4)} H_{u} \\
& +y_{3}^{u} c^{c} Q_{3} Y_{1}^{(6)} H_{u}+y_{4}^{u} c^{c} Q_{D} Y_{2}^{(3)} H_{u}+y_{5}^{u} t^{c} Q_{3}, \\
\mathcal{W}_{d}= & y_{1}^{d} d^{c} Q_{D} Y_{2}^{(5)} H_{d}+y_{2}^{d} s^{c} Q_{D} Y_{\mathbf{2}^{\prime \prime}}^{(3)} H_{d}+y_{3}^{d} b^{c} Q_{3} H_{d},
\end{aligned}
$$

where all coupling constants except $y_{4}^{u}$ can be taken to be real by using the freedom of field redefinition. We can write the up- and down-quark mass matrices as follows:

$$
\begin{aligned}
M_{u} & =\left(\begin{array}{ccc}
y_{1}^{u} Y_{\mathbf{2}, 2}^{(1)} & -y_{1}^{u} Y_{\mathbf{2}, 1}^{(1)} & y_{2}^{u} Y_{\mathbf{1}}^{(4)} \\
y_{3}^{u} Y_{\mathbf{2}, 2}^{(3)} & -y_{3}^{u} Y_{\mathbf{2}, 1}^{(3)} & y_{4}^{u} Y_{\mathbf{1}}^{(6)} \\
0 & 0 & y_{5}^{u}
\end{array}\right) v_{u}, \\
M_{d} & =\left(\begin{array}{ccc}
y_{1}^{d} Y_{\mathbf{2}, 2}^{(5)} & -y_{1}^{d} Y_{\mathbf{2}, 1}^{(5)} & 0 \\
y_{2}^{d} Y_{\mathbf{2}^{\prime \prime}, 2}^{(3)} & -y_{2}^{d} Y_{\mathbf{2}^{\prime \prime}, 1}^{(3)} & 0 \\
0 & 0 & y_{3}^{d}
\end{array}\right) v_{d} .
\end{aligned}
$$

We see that $M_{u}$ and $M_{d}$ are expressed in terms of 11 free input parameters: $y_{1,2,3,5}^{u},\left|y_{4}^{u}\right|, \arg \left(y_{4}^{u}\right), y_{1,2,3}^{d}, \operatorname{Re} \tau$, and $\operatorname{Im} \tau$.

Model IV: Five zero elements and 11 free parameters

The classification of the quark fields under the $T^{\prime}$ modular symmetry is the same as that of Model III, while the assignments for the modular weights are different, as shown in Table V. The superpotential for quark masses is of the following form:

$$
\begin{aligned}
\mathcal{W}_{u}= & y_{1}^{u} u^{c} Q_{D} Y_{2}^{(1)} H_{u}+y_{2}^{u} u^{c} Q_{3} Y_{1}^{(4)} H_{u} \\
& +y_{3}^{u} c^{c} Q_{3} Y_{1}^{(6)} H_{u}+y_{4}^{u} c^{c} Q_{D} Y_{2}^{(3)} H_{u} \\
& +y_{5}^{u} t^{c} Q_{D} Y_{2}^{(5)} H_{u}, \\
\mathcal{W}_{d}= & y_{1}^{d} d^{c} Q_{D} Y_{2}^{(5)} H_{d}+y_{2}^{d} s^{c} Q_{D} Y_{\mathbf{2}^{\prime \prime}}^{(3)} H_{d}+y_{3}^{d} b^{c} Q_{3} H_{d},
\end{aligned}
$$

where all coefficients except $y_{4}^{u}$ can be taken to be real and positive. Using the decomposition rules of the $T^{\prime}$ group in Appendix A, we obtain

$$
\begin{aligned}
& M_{u}=\left(\begin{array}{ccc}
y_{1}^{u} Y_{\mathbf{2}, 2}^{(1)} & -y_{1}^{u} Y_{\mathbf{2}, 1}^{(1)} & y_{2}^{u} Y_{\mathbf{1}}^{(4)} \\
y_{3}^{u} Y_{\mathbf{2}, 2}^{(3)} & -y_{3}^{u} Y_{\mathbf{2}, 1}^{(3)} & y_{4}^{u} Y_{\mathbf{1}}^{(6)} \\
y_{5}^{u} Y_{\mathbf{2}, 2}^{(5)} & -y_{5}^{u} Y_{\mathbf{2}, 1}^{(5)} & 0
\end{array}\right) v_{u}, \\
& M_{d}=\left(\begin{array}{ccc}
y_{1}^{d} Y_{\mathbf{2}, 2}^{(5)} & -y_{1}^{d} Y_{\mathbf{2}, 1}^{(5)} & 0 \\
y_{2}^{d} Y_{\mathbf{2}^{\prime \prime}, 2}^{(3)} & -y_{2}^{d} Y_{\mathbf{2}^{\prime \prime}, 1}^{(3)} & 0 \\
0 & 0 & y_{3}^{d}
\end{array}\right) v_{d},
\end{aligned}
$$

which involve 11 free real parameters including the real and imaginary parts of the modulus $\tau$.

Model V: Four zero elements and 11 free parameters

In this model, both left-handed and right-handed quark fields are assigned to transform as the direct sum of doublet and singlet representations of $T^{\prime}$, i.e., $Q_{D} \sim \mathbf{2}, \quad Q_{3} \sim \mathbf{1}, \quad u_{D}^{c} \equiv\left(u^{c}, c^{c}\right) \sim \mathbf{2}^{\prime \prime}, \quad t^{c} \sim \mathbf{1}^{\prime \prime}$, $d_{D}^{c} \equiv\left(d^{c}, s^{c}\right) \sim \mathbf{2}^{\prime}$, and $b^{c} \sim \mathbf{1}$. The modular-invariant superpotential in the quark sector is

$$
\begin{aligned}
\mathcal{W}_{u}= & y_{1}^{u} u_{D}^{c} Q_{D} Y_{\mathbf{3}}^{(2)} H_{u}+y_{2}^{u} u_{D}^{c} Q_{3} Y_{\mathbf{2}}^{(5)} H_{u} \\
& +y_{3}^{u} t^{c} Q_{D} Y_{\mathbf{2}}^{(1)} H_{u}+y_{4}^{u} t^{c} Q_{3} Y_{\mathbf{1}^{\prime}}^{(4)} H_{u}, \\
\mathcal{W}_{d}= & y_{1}^{d} d_{D}^{c} Q_{D} Y_{\mathbf{3}}^{(6)} H_{d}+y_{2}^{d} d_{D}^{c} Q_{D} Y_{\mathbf{3} I}^{(6)} H_{d}+y_{3}^{d} b^{c} Q_{3} H_{d},
\end{aligned}
$$

where the coupling constants $y_{1,2,4}^{u}$ and $y_{1,3}^{d}$ can be taken to be real and positive without loss of generality, while the phases of $y_{3}^{u}$ and $y_{2}^{d}$ cannot be absorbed into quark fields. The up- and down-quark mass matrices are given as

$$
\begin{aligned}
M_{u} & =\left(\begin{array}{ccc}
-\sqrt{2} e^{\frac{5 i \pi}{12}} y_{1}^{u} Y_{\mathbf{3}, 3}^{(2)} & y_{1}^{u} Y_{\mathbf{3}, 1}^{(2)} & -y_{2}^{u} Y_{\mathbf{2}, 2}^{(5)} \\
y_{1}^{u} Y_{\mathbf{3}, 1}^{(2)} & -\sqrt{2} e^{\frac{7 \pi \pi}{12}} y_{1}^{u} Y_{\mathbf{3}, 2}^{(2)} & y_{2}^{u} Y_{\mathbf{2}, 1}^{(5)} \\
y_{3}^{u} Y_{\mathbf{2}, 2}^{(1)} & -y_{3}^{u} Y_{\mathbf{2}, 1}^{(1)} & y_{4}^{u} Y_{\mathbf{1}^{\prime}}^{(4)}
\end{array}\right) v_{u}, \\
M_{d} & =\left(\begin{array}{ccc}
\sqrt{2} e^{\frac{5 i \pi}{12}}\left(y_{1}^{d} Y_{\mathbf{3} I, 1}^{(6)}+y_{2}^{d} Y_{\mathbf{3} I I, 1}^{(6)}\right) & -y_{1}^{d} Y_{\mathbf{3} I, 2}^{(6)}-y_{2}^{d} Y_{\mathbf{3} I I, 2}^{(6)} & 0 \\
-y_{1}^{d} Y_{\mathbf{3}, 2}^{(6)}-y_{2}^{d} Y_{\mathbf{3} I I, 2}^{(6)} & \sqrt{2} e^{\frac{7 i \pi}{12}}\left(y_{1}^{d} Y_{\mathbf{3} I, 3}^{(6)}+y_{2}^{d} Y_{\mathbf{3} I I, 3}^{(6)}\right) & 0 \\
0 & 0 & y_{3}^{d}
\end{array}\right) v_{d} .
\end{aligned}
$$




\section{A. Numerical results}

In previous work, the texture-zero structure of the quark mass matrices was an assumption or the zero entry was highly suppressed when imposing certain flavor symmetries, and the mass matrix was assumed to be symmetric or Hermitian. In the present formalism, the texture zero is exactly dictated by the modular symmetry, and the nonvanishing entries are correlated. Therefore, modular symmetry provides a natural framework to realize texture zeros. Moreover, it is remarkable that the down-quark mass matrix $M_{d}$ is block diagonal, where the (13), (23), (31), and (32) elements of $M_{d}$ vanish exactly for all five models summarized in Table V. As a consequence, the small offdiagonal entries $V_{u b}, V_{c b}, V_{t d}$, and $V_{t s}$ of the CKM matrix arise entirely from the up-type quark sector.

The above predictions for the up- and down-quark mass matrices $M_{u}$ and $M_{d}$ in Eqs. (84), (57), (59), (61), and (63) are given at the scale where the modulus $\tau$ obtains a vacuum expectation value. Here we assume that the flavorsymmetry-breaking scale is very large, around the grand unified theory (GUT) scale $2 \times 10^{16} \mathrm{GeV}$. For each set of given values of the coupling constants and the modulus $\tau$, we numerically diagonalize $M_{u}$ and $M_{d}$, and subsequently extract the predictions for quark masses and the CKM mixing matrix which may be compared with their GUTscale values. It is well known that the quark masses and mixing parameters at the GUT scale can be obtained from the values measured in low-energy experiments by performing renormalization group evolution (RGE). The running of best-fit and error values to the GUT scale is generally dependent on supersymmetry (SUSY) parameters, primarily the SUSY-breaking scale $M_{\mathrm{SUSY}}$ and $\tan \beta$. In our numerical analysis, we extract the GUT-scale values of all Yukawa couplings and the CKM parameters from Ref. [77], assuming a SUSY-breaking scale $M_{\mathrm{SUSY}}=$ $10 \mathrm{TeV}$ and $\tan \beta=10$. The central values as well as the $1 \sigma$ uncertainty ranges of different observables used in our analysis are listed in Table VI.

Following Ref. [11] and other papers in the literature, we treat the $\mathrm{VEV}$ of the complex modulus $\tau$ as a free parameter. The modulus value is expected to be dynamically fixed as a minimum of the scalar potential in supergravity theory. The modulus stabilization could be achieved in $\operatorname{SL}(2, Z)$ modular-invariant theories after the nonperturbative superpotential in supergravity theory is taken into account [78,79]. It has been shown that modulus stabilization can also be realized in the bottom-up modular symmetry models in a similar manner [46].

In order to determine the values of the input parameters that best fit the experimental data, we define a $\chi^{2}$ function

$$
\chi^{2}=\sum_{i=1}^{n}\left(\frac{P_{i}\left(x_{1}, x_{2}, \ldots, x_{m}\right)-\mu_{i}}{\sigma_{i}}\right)^{2},
$$

TABLE VI. The best-fit values $\mu_{i}$ and $1 \sigma$ uncertainties of the quark and lepton parameters when evolved to the GUT scale as calculated in Ref. [77], with the SUSY breaking scale $M_{\mathrm{SUSY}}=$ $10 \mathrm{TeV}$ and $\tan \beta=10$, where the error widths represent $1 \sigma$ intervals. The quark masses are given as $m_{u, c, t}=y_{u, c, t} v_{u}$ and $m_{d, s, b}=y_{d, s, b} v_{d}$. The values of lepton mixing angles, leptonic Dirac $C P$ violation phases $\delta_{C P}^{l}$, and the neutrino mass-squared difference are taken from NuFit 4.1 [1].

\begin{tabular}{lc}
\hline \hline Parameters & $\mu_{i} \pm 1 \sigma$ \\
\hline$y_{u} / 10^{-6}$ & $2.73325 \pm 0.84731$ \\
$y_{c} / 10^{-3}$ & $1.41719 \pm 0.04960$ \\
$y_{t}$ & $0.50232 \pm 0.01200$ \\
$y_{d} / 10^{-6}$ & $5.12495 \pm 0.56374$ \\
$y_{s} / 10^{-4}$ & $1.01438 \pm 0.05478$ \\
$y_{b} / 10^{-3}$ & $5.56096 \pm 0.06103$ \\
$y_{e} / 10^{-6}$ & $2.07526 \pm 0.01245$ \\
$y_{\mu} / 10^{-4}$ & $4.38107 \pm 0.02629$ \\
$y_{\tau} / 10^{-3}$ & $7.48026 \pm 0.03898$ \\
$\theta_{12}^{q}$ & $0.22736 \pm 0.00073$ \\
$\theta_{13}^{q} / 10^{-2}$ & $0.34938 \pm 0.01258$ \\
$\theta_{23}^{q}$ & $0.04015 \pm 0.00064$ \\
$\delta_{C P}^{q} /{ }^{\circ}$ & $69.21330 \pm 3.11460$ \\
$\sin ^{2} \theta_{12}^{l}$ & $0.310_{-0.012}^{+0.013}$ \\
$\sin ^{2} \theta_{23}^{l}$ & $0.563_{-0.014}^{+0.018}$ \\
$\sin ^{2} \theta_{13}^{l}$ & $0.02237_{-0.00065}^{+0.0066}$ \\
$\delta_{C P}^{l} /{ }^{\circ}$ & $221_{-28}^{+39}$ \\
$\Delta m_{21}^{2}$ & $7.39_{-0.20}^{+0.21}$ \\
\hline $0^{-5} \mathrm{eV}^{2}$ & $2.528_{-0.031}^{+0.029}$ \\
$m_{31}^{2}$ & \\
\hline \hline $0^{-3} \mathrm{eV}^{2}$ & \\
\hline & \\
\hline
\end{tabular}

where $P_{i}$ are predictions for the physical observables derived from the up-type and down-type quark mass matrices $M_{u}$ and $M_{d}$ as complex nonlinear functions of the free parameters of the model, and $\mu_{i}$ and $\sigma_{i}$ are the GUTscale central values and $1 \sigma$ deviations, respectively, of the corresponding quantities listed in Table VI. The measured values of the top- and bottom-quark masses can be reproduced exactly by properly choosing the values of the overall parameters $y_{1}^{u} v_{u}$ and $y_{1}^{d} v_{d}$. Hence, we include the mass ratios $m_{u} / m_{c}, m_{c} / m_{t}, m_{d} / m_{s}$, and $m_{s} / m_{b}$ instead of quark masses $m_{u, c, t}$ and $m_{d, s, b}$ individually. We take the complex modulus $\tau$ as random complex number in the fundamental domain $\{\tau:|\operatorname{Re} \tau| \leq 1 / 2, \operatorname{Im} \tau>0,|\tau| \geq 1\}$. The absolute values of all coupling constants are scanned in the region $\left[0,10^{6}\right]$, while the phases are freely varied in the range $[0,2 \pi]$. The function $\chi^{2}$ is numerically minimized by using the minimization algorithms included in the package MINUIT developed by CERN to determine the best-fit values of the input parameters. From the best-fit values of the input parameters, one can obtain the corresponding values of quark masses and the CKM mixing matrix.

As shown in Sec. V, both Model I and Model II have ten free parameters and the remaining Models III-V have 
TABLE VII. The best-fit values of the input parameters, quark mass ratios, and the CKM parameters for the five models summarized in Table V, where Obs denotes the observables. The fit is performed for the GUT-scale extrapolated data given in Table VI. Notice that the measured values of the top-quark mass $m_{t}$ and bottom-quark mass $m_{b}$ are reproduced exactly here.

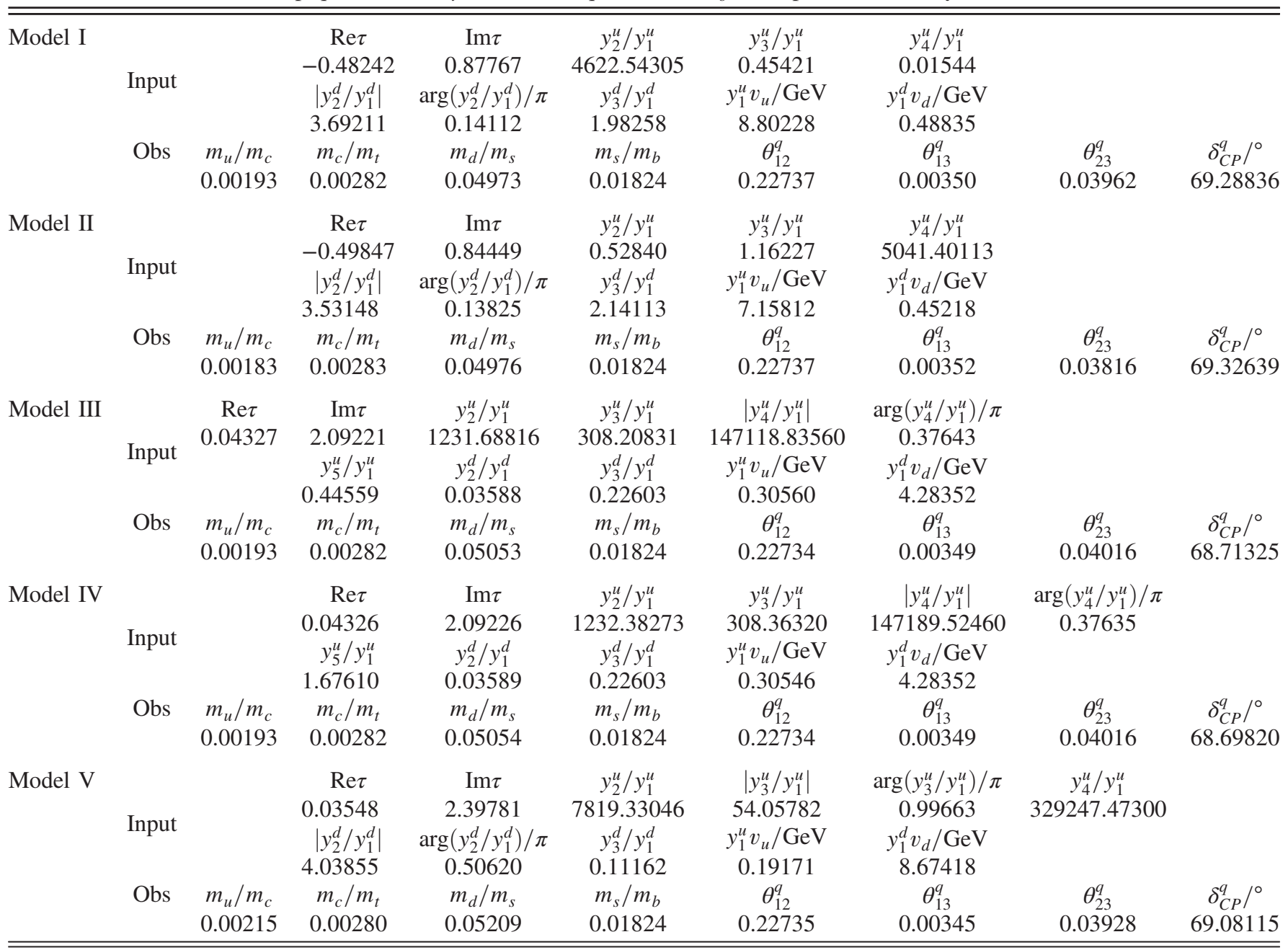

11 free parameters. We fit all 10 or 11 free parameters of each model using the ten observables, including six quark masses $m_{u, c, t}, m_{d, s, b}$, three quark mixing angles $\theta_{12}^{q}, \theta_{13}^{q}$, $\theta_{23}^{q}$, and one quark $C P$ violation phase $\delta_{C P}^{q}$. The results of the fitting are shown in Table VII. We see that all five models can give very good fits to the data, and the resulting quark masses and mixing parameters all lie within their $1 \sigma$ ranges. When extending these models to include the lepton sector in the following section, we fix the complex modulus $\tau$ to be the best-fit values in Table VII which are determined from the precisely measured quark masses and CKM mixing matrix.

\section{QUARK-LEPTON UNIFICATION}

Inspired by the success of $T^{\prime}$ modular symmetry in explaining the quark masses and CKM mixing matrix, we extend this framework to the lepton sector. Since the solar mixing angle $\theta_{12}^{l}$ and atmospheric mixing angle $\theta_{23}^{l}$ are large, and the reactor mixing angle $\theta_{13}^{l}$ is of the same order as the Cabibbo angle, the lepton mixing matrix does not have a hierarchical structure. Therefore, we will not use the doublet representations of $T^{\prime}$ to distinguish the first two generations of leptons from the third generation. The three generations of left-handed lepton doublets $L=$ $\left(L_{1}, L_{2}, L_{3}\right)^{T}$ are assigned to be in a triplet 3 under $T^{\prime}$, while the right-handed charged leptons $e^{c}, \mu^{c}$, and $\tau^{c}$ are assumed to transform as singlet representations $\mathbf{1}, \mathbf{1}^{\prime}$, or $\mathbf{1}^{\prime \prime}$ of the $T^{\prime}$ modular group. For the irreducible representations $\mathbf{3}, \mathbf{1}, \mathbf{1}^{\prime}$, and $\mathbf{1}^{\prime \prime}$, two distinct elements of $T^{\prime}$ group are described by the same matrices that represent the elements in $A_{4}$. Therefore, the group $T^{\prime}$ cannot be distinguished from $A_{4}$ when working with these representations. As a consequence, the flavor symmetry is essentially the $A_{4}$ modular group in the lepton sector. A systematic classification of lepton models with $A_{4}$ modular symmetry was performed in Ref. [43]. In the present work, we focus on two economical models, which were called $\mathcal{D}_{6}$ and $\mathcal{D}_{9}$ in 
TABLE VIII. The transformation properties of the lepton chiral superfields under the standard model gauge group $S U(2)_{L} \times$ $U(1)_{Y}$ and under $T^{\prime}$ modular symmetry for the models $\mathcal{D}_{6}$ and $\mathcal{D}_{9}$ of Ref. [43], where $-k_{I}$ refers to the modular weights.

\begin{tabular}{lcccccc}
\hline \hline & $L$ & $e^{c}$ & $\mu^{c}$ & $\tau^{c}$ & $N^{c}$ \\
\hline$S U(2)_{L} \times U(1)_{Y}$ & $(2,-1 / 2)$ & $(1,1)$ & $(1,1)$ & $(1,1)$ & $(1,0)$ \\
\hline $\mathcal{D}_{6}$ & $T^{\prime}$ & $\mathbf{3}$ & $\mathbf{1}^{\prime}$ & $\mathbf{1}^{\prime}$ & $\mathbf{1}$ & $\mathbf{3}$ \\
& $k_{I}$ & 1 & 1 & 3 & 1 & 1 \\
$\mathcal{D}_{9}$ & $T^{\prime}$ & $\mathbf{3}$ & $\mathbf{1}^{\prime \prime}$ & $\mathbf{1}^{\prime \prime}$ & $\mathbf{1}^{\prime}$ & $\mathbf{3}$ \\
& $k_{I}$ & 1 & 1 & 3 & 1 & 1 \\
\hline \hline
\end{tabular}

Ref. [43]. The transformation properties of the lepton chiral superfields and the right-handed neutrinos $N^{c}=$ $\left(N_{1}^{c}, N_{2}^{c}, N_{3}^{c}\right)^{T}$ under $T^{\prime}$ and their modular weights are summarized in Table VIII. Note that the Higgs doublets $H_{u, d}$ transform trivially under $T^{\prime}$ with zero modular weight.

\section{A. Lepton sector}

In the following, we present the neutrino and charged lepton mass terms and the corresponding lepton mass matrices for the models $\mathcal{D}_{6}$ and $\mathcal{D}_{9}$. The neutrino masses are assumed to be generated from the type-I seesaw mechanism. As listed in Table VIII, the right-handed neutrinos $N^{c}$ compose a $T^{\prime}$ triplet $\mathbf{3}$.

(1) $\mathcal{D}_{6}$ lepton model

In this case, the modular-invariant superpotential in the lepton sector reads

$$
\begin{aligned}
\mathcal{W}_{e}= & \alpha e^{c}\left(L Y_{\mathbf{3}}^{(2)}\right)_{\mathbf{1}^{\prime \prime}} H_{d}+\beta \mu^{c}\left(L Y_{\mathbf{3}}^{(4)}\right)_{\mathbf{1}^{\prime \prime}} H_{d} \\
& +\gamma \tau^{c}\left(L Y_{\mathbf{3}}^{(2)}\right)_{\mathbf{1}} H_{d}, \\
\mathcal{W}_{\nu}= & g_{1}\left(\left(N^{c} L\right)_{\mathbf{3}_{S}} Y_{\mathbf{3}}^{(2)}\right)_{\mathbf{1}} H_{u} \\
& +g_{2}\left(\left(N^{c} L\right)_{\mathbf{3}_{A}} Y_{\mathbf{3}}^{(2)}\right)_{\mathbf{1}} H_{u}+\Lambda\left(\left(N^{c} N^{c}\right)_{\mathbf{3}_{S}} Y_{\mathbf{3}}^{(2)}\right)_{\mathbf{1}} .
\end{aligned}
$$

The resulting charged lepton mass matrix $M_{e}$, the Dirac neutrino mass matrix $M_{D}$, and the Majorana mass matrix $M_{N}$ for heavy neutrinos are given by

$$
\begin{aligned}
M_{e}= & \left(\begin{array}{ccc}
\alpha Y_{\mathbf{3}, 3}^{(2)} & \alpha Y_{\mathbf{3}, 2}^{(2)} & \alpha Y_{\mathbf{3}, 1}^{(2)} \\
\beta Y_{\mathbf{3}, 3}^{(4)} & \beta Y_{\mathbf{3}, 2}^{(4)} & \beta Y_{\mathbf{3}, 1}^{(4)} \\
\gamma Y_{\mathbf{3}, 1}^{(2)} & \gamma Y_{\mathbf{3}, 3}^{(2)} & \gamma Y_{\mathbf{3}, 2}^{(2)}
\end{array}\right) v_{d}, \quad M_{N}=\left(\begin{array}{rrr}
2 Y_{\mathbf{3}, 1}^{(2)} & -Y_{\mathbf{3}, \mathbf{3}}^{(2)} & -Y_{\mathbf{3}, 2}^{(2)} \\
-Y_{\mathbf{3}, 3}^{(2)} & 2 Y_{\mathbf{3}, 2}^{(2)} & -Y_{\mathbf{3}, 1}^{(2)} \\
-Y_{\mathbf{3}, 2}^{(2)} & -Y_{\mathbf{3}, 1}^{(2)} & 2 Y_{\mathbf{3}, 3}^{(2)}
\end{array}\right) \Lambda, \\
M_{D}= & \left(\begin{array}{ccc}
2 g_{1} Y_{\mathbf{3}, 1}^{(2)} & \left(-g_{1}+g_{2}\right) Y_{\mathbf{3}, 3}^{(2)} & \left(-g_{1}-g_{2}\right) Y_{\mathbf{3}, 2}^{(2)} \\
\left(-g_{1}-g_{2}\right) Y_{\mathbf{3}, 3}^{(2)} & 2 g_{1} Y_{\mathbf{3}, 2}^{(2)} & \left(-g_{1}+g_{2}\right) Y_{\mathbf{3}, 1}^{(2)} \\
\left(-g_{1}+g_{2}\right) Y_{\mathbf{3}, 2}^{(2)} & \left(-g_{1}-g_{2}\right) Y_{\mathbf{3}, 1}^{(2)} & 2 g_{1} Y_{\mathbf{3}, 3}^{(2)}
\end{array}\right) v_{u} .
\end{aligned}
$$

The light neutrino mass matrix is given by the seesaw formula $m_{\nu}=-M_{D}^{T} M_{N}^{-1} M_{D}$. The phases of the parameters $\alpha, \beta$, and $\gamma$ can be absorbed into the right-handed charged lepton fields, and the measured charged lepton masses can be reproduced by adjusting their values. The light neutrino mass matrix $m_{\nu}$ only depends on the complex parameter $g_{2} / g_{1}$ and modulus $\tau$, besides the overall scale $g_{1}^{2} v_{u}^{2} / \Lambda$. We find that the modulus $\tau$ lies in narrow regions in order to be compatible with the experimental data on lepton mixing angles and neutrino masses [43].

(2) $\mathcal{D}_{9}$ lepton model

From Table VIII, we see that the models $\mathcal{D}_{6}$ and $\mathcal{D}_{9}$ differ in the representation assignments for the right-handed charged leptons, while the transformation properties of $L$ and $N^{c}$ are exactly the same in the two models. The superpotential for the charged lepton masses is given by

$$
\begin{aligned}
\mathcal{W}_{e}= & \alpha e^{c}\left(L Y_{\mathbf{3}}^{(2)}\right)_{\mathbf{1}^{\prime}} H_{d}+\beta \mu^{c}\left(L Y_{\mathbf{3}}^{(4)}\right)_{\mathbf{1}^{\prime}} H_{d} \\
& +\gamma \tau^{c}\left(L Y_{\mathbf{3}}^{(2)}\right)_{\mathbf{1}^{\prime \prime}} H_{d}
\end{aligned}
$$

which leads to the following charged lepton mass matrix:

$$
M_{e}=\left(\begin{array}{lll}
\alpha Y_{\mathbf{3}, 2}^{(2)} & \alpha Y_{\mathbf{3}, 1}^{(2)} & \alpha Y_{\mathbf{3}, 3}^{(2)} \\
\beta Y_{\mathbf{3}, 2}^{(4)} & \beta Y_{\mathbf{3}, 1}^{(4)} & \beta Y_{\mathbf{3}, 3}^{(4)} \\
\gamma Y_{\mathbf{3}, 3}^{(2)} & \gamma Y_{\mathbf{3}, 2}^{(2)} & \gamma Y_{\mathbf{3}, 1}^{(2)}
\end{array}\right)
$$

The superpotential $\mathcal{W}_{\nu}$ for the neutrino sector coincides with that of Eq. (65), and the relevant neutrino mass matrices $M_{D}$ and $M_{N}$ are given by Eq. (66). 


\section{B. Numerical results}

We can combine the benchmark quark models I-V presented in Sec. $\mathrm{V}$ with the $\mathcal{D}_{6}$ and $\mathcal{D}_{9}$ lepton models to give a unified description of both the quark and lepton sectors. Notice that the modulus $\tau$ in the quark and lepton mass matrices should be identical, and we transfer the bestfit value of $\tau$ in Table VII obtained from the quark sector to the lepton sector. We perform a global fit to the complete models including both quark and lepton sectors, the models would be viable if the observed quark and lepton masses and mixing parameters can be accommodated for certain values of input parameters. It is remarkable that the resulting models have fewer free input parameters than the number of observable quantities, including quark and lepton masses and mixing parameters. Hence, it is highly nontrivial that the model can successfully fit the data. In particular, the light neutrino mass matrix would only depend on a complex parameter $g_{2} / g_{1}$ and a overall factor $g_{1}^{2} v_{u}^{2} / \Lambda$. Consequently, three light neutrino masses, three lepton mixing angles, and three $C P$ violation phases are determined using three real input parameters: $\left|g_{2} / g_{1}\right|$, $\arg \left(g_{2} / g_{1}\right)$, and $g_{1}^{2} v_{u}^{2} / \Lambda$. Thus, the models could make six nontrivial predictions that agree well with the present data. The extrapolated values of the charged lepton masses at the GUT scale are listed in Table VI for $\tan \beta=10$ and $M_{\text {SUSY }}=10 \mathrm{TeV}$. For the neutrino masses and mixing angles, we use the latest results of the global data analysis from NuFit 4.1 [1], and the RGE effects from the low scale to the GUT scale are neglected, since the running of the neutrino masses and mixing angles in the minimal supersymmetric SM is known to be negligible in the case of $\tan \beta \leq 30$. We find that the RGE dependence can really be safely neglected in a sizable region of the $\tan \beta-M_{\text {SUSY }}$ plane [35].

Since the global analysis of neutrino oscillation data favors normal mass ordering over inverted ordering [1], we assume a normal ordering of neutrino masses in the $\chi^{2}$

TABLE IX. The best-fit values of the input parameters, the charged lepton mass ratios, the light neutrino masses, and the lepton mixing parameters for the quark-lepton unified models, where Obs denotes the observables. The modulus $\tau$ is taken to be the best-fit values in Table VII which give rise to realistic values of quark masses and the CKM mixing matrix. The fit is performed for the GUTscale extrapolated data given in Table VI. Notice that the measured values of the tau lepton mass $m_{\tau}$ are reproduced exactly here.

\begin{tabular}{|c|c|c|c|c|c|c|c|}
\hline Model I $+\mathcal{D}_{6}$ & Input & $\begin{array}{c}\beta / \alpha \\
0.37455 \\
m_{e} / m_{\mu} \\
0.00474 \\
\sin ^{2} \theta_{12}^{l} \\
0.31002\end{array}$ & $\begin{array}{c}\gamma / \alpha \\
0.00446 \\
m_{\mu} / m_{\tau} \\
0.05857 \\
\sin ^{2} \theta_{13}^{l} \\
0.02236\end{array}$ & $\begin{array}{c}\left|g_{2} / g_{1}\right| \\
1.11410 \\
m_{1} / \mathrm{eV} \\
0.13241 \\
\sin ^{2} \theta_{23}^{l} \\
0.54645\end{array}$ & $\begin{array}{c}\arg \left(g_{2} / g_{1}\right) / \pi \\
1.62831 \\
m_{2} / \mathrm{eV} \\
0.13269 \\
\delta_{C P}^{l} /^{\circ} \\
277.83689\end{array}$ & $\begin{array}{c}\alpha v_{d} / \mathrm{GeV} \\
8.34476 \\
m_{3} / \mathrm{eV} \\
0.14164 \\
\alpha_{21} /{ }^{\circ} \\
359.49213\end{array}$ & $\begin{array}{c}\left(g_{1}^{2} v_{u}^{2} / \Lambda\right) / \mathrm{eV} \\
0.42538 \\
\left|m_{e e}\right| / \mathrm{eV} \\
0.13258 \\
\alpha_{31} /^{\circ} \\
179.10767\end{array}$ \\
\hline Model II $+\mathcal{D}_{6}$ & $\begin{array}{l}\text { Input } \\
\text { Obs }\end{array}$ & $\begin{array}{c}\beta / \alpha \\
0.36049 \\
m_{e} / m_{\mu} \\
0.00474 \\
\sin ^{2} \theta_{12}^{l} \\
0.30998\end{array}$ & $\begin{array}{c}\gamma / \alpha \\
0.00427 \\
m_{\mu} / m_{\tau} \\
0.05857 \\
\sin ^{2} \theta_{13}^{l} \\
0.02238\end{array}$ & $\begin{array}{c}\left|g_{2} / g_{1}\right| \\
1.11554 \\
m_{1} / \mathrm{eV} \\
0.12929 \\
\sin ^{2} \theta_{23}^{l} \\
0.54777\end{array}$ & $\begin{array}{c}\arg \left(g_{2} / g_{1}\right) / \pi \\
1.37166 \\
m_{2} / \mathrm{eV} \\
0.12958 \\
\delta_{C P}^{l} /^{\circ} \\
261.65953\end{array}$ & $\begin{array}{c}\alpha v_{d} / \mathrm{GeV} \\
8.02910 \\
m_{3} / \mathrm{eV} \\
0.13872 \\
\alpha_{21} /{ }^{\circ} \\
0.55957\end{array}$ & $\begin{array}{c}\left(g_{1}^{2} v_{u}^{2} / \Lambda\right) / \mathrm{eV} \\
0.39971 \\
\left|m_{e e}\right| / \mathrm{eV} \\
0.12945 \\
\alpha_{31} /{ }^{\circ} \\
180.62437\end{array}$ \\
\hline Model III $+\mathcal{D}_{6}$ & $\begin{array}{l}\text { Input } \\
\text { Obs }\end{array}$ & $\begin{array}{c}\beta / \alpha \\
4.32234 \\
m_{e} / m_{\mu} \\
0.00474 \\
\sin ^{2} \theta_{12}^{l} \\
0.31001\end{array}$ & $\begin{array}{c}\gamma / \alpha \\
0.00031 \\
m_{\mu} / m_{\tau} \\
0.05858 \\
\sin ^{2} \theta_{13}^{l} \\
0.02237\end{array}$ & $\begin{array}{c}\left|g_{2} / g_{1}\right| \\
1.15601 \\
m_{1} / \mathrm{eV} \\
0.07922 \\
\sin ^{2} \theta_{23}^{l} \\
0.57919\end{array}$ & $\begin{array}{c}\arg \left(g_{2} / g_{1}\right) / \pi \\
1.37529 \\
m_{2} / \mathrm{eV} \\
0.07969 \\
\delta_{C P}^{l} /{ }^{\circ} \\
250.41152\end{array}$ & $\begin{array}{c}\alpha v_{d} / \mathrm{GeV} \\
10.55424 \\
m_{3} / \mathrm{eV} \\
0.09383 \\
\alpha_{21} /^{\circ} \\
1.45635\end{array}$ & $\begin{array}{c}\left(g_{1}^{2} v_{u}^{2} / \Lambda\right) / \mathrm{eV} \\
0.35880 \\
\left|m_{e e}\right| / \mathrm{eV} \\
0.07921 \\
\alpha_{31} /^{\circ} \\
181.26500\end{array}$ \\
\hline Model IV $+\mathcal{D}_{6}$ & $\begin{array}{l}\text { Input } \\
\text { Obs }\end{array}$ & $\begin{array}{c}\beta / \alpha \\
4.32332 \\
m_{e} / m_{\mu} \\
0.00474 \\
\sin ^{2} \theta_{12}^{l} \\
0.31001\end{array}$ & $\begin{array}{c}\gamma / \alpha \\
0.00031 \\
m_{\mu} / m_{\tau} \\
0.05858 \\
\sin ^{2} \theta_{13}^{l} \\
0.02238\end{array}$ & $\begin{array}{c}\left|g_{2} / g_{1}\right| \\
1.15603 \\
m_{1} / \mathrm{eV} \\
0.07923 \\
\sin ^{2} \theta_{23}^{l} \\
0.57917\end{array}$ & $\begin{array}{c}\arg \left(g_{2} / g_{1}\right) / \pi \\
1.37526 \\
m_{2} / \mathrm{eV} \\
0.07969 \\
\delta_{C P}^{l} /^{\circ} \\
250.41813\end{array}$ & $\begin{array}{c}\alpha v_{d} / \mathrm{GeV} \\
10.55380 \\
m_{3} / \mathrm{eV} \\
0.09383 \\
\alpha_{21} /^{\circ} \\
1.45589\end{array}$ & $\begin{array}{c}\left(g_{1}^{2} v_{u}^{2} / \Lambda\right) / \mathrm{eV} \\
0.35881 \\
\left|m_{e e}\right| / \mathrm{eV} \\
0.07921 \\
\alpha_{31} /^{\circ} \\
181.26512\end{array}$ \\
\hline Model $\mathrm{V}+\mathcal{D}_{9}$ & $\begin{array}{l}\text { Input } \\
\text { Obs }\end{array}$ & $\begin{array}{c}\beta / \alpha \\
149.62275 \\
m_{e} / m_{\mu} \\
0.00474 \\
\sin ^{2} \theta_{12}^{l} \\
0.30988\end{array}$ & $\begin{array}{c}\gamma / \alpha \\
284.15784 \\
m_{\mu} / m_{\tau} \\
0.05857 \\
\sin ^{2} \theta_{13}^{l} \\
0.02241\end{array}$ & $\begin{array}{c}\left|g_{2} / g_{1}\right| \\
1.12239 \\
m_{1} / \mathrm{eV} \\
0.11598 \\
\sin ^{2} \theta_{23}^{l} \\
0.55773\end{array}$ & $\begin{array}{c}\arg \left(g_{2} / g_{1}\right) / \pi \\
0.37191 \\
m_{2} / \mathrm{eV} \\
0.11630 \\
\delta_{C P}^{l} /^{\circ} \\
259.82911\end{array}$ & $\begin{array}{c}\alpha v_{d} / \mathrm{GeV} \\
0.04122 \\
m_{3} / \mathrm{eV} \\
0.12641 \\
\alpha_{21} /^{\circ} \\
0.68898\end{array}$ & $\begin{array}{c}\left(g_{1}^{2} v_{u}^{2} / \Lambda\right) / \mathrm{eV} \\
0.52347 \\
\left|m_{e e}\right| / \mathrm{eV} \\
0.11613 \\
\alpha_{31} /^{\circ} \\
180.73465\end{array}$ \\
\hline
\end{tabular}


analysis. Similar to Sec. V A, the absolute value of each coupling constant freely varies between 0 and $10^{6}$, all phases are treated as random numbers in the range from 0 to $2 \pi$, and the complex modulus $\tau$ is scanned in the fundamental domain of the modular group. The results of the fit are shown in Table IX. We see that a very good fit to the data is obtained, and all observables from the model fall well within the experimentally allowed regions. From the fitted values of the input parameters, we can further obtain predictions for the unmeasured observables, such as the Dirac $C P$ phase $\delta_{C P}^{l}$ and Majorana $C P$ phases $\alpha_{21}$ and $\alpha_{31}$ in the lepton sector, the lightest neutrino mass $m_{1}$, and the effective Majorana mass $\left|m_{e e}\right|$ in neutrinoless double-beta decay, as shown in Table IX.

As an example, we consider the scenario where the Model I in quark sector is combined with the lepton model $\mathcal{D}_{6}$. We have 16 real input parameters $\left|y_{1,2,3,4}^{u}\right|,\left|y_{1,3}^{d}\right|,\left|y_{2}^{d}\right|$, $\arg \left(y_{2}^{d}\right),|\alpha|,|\beta|,|\gamma|,\left|g_{1}\right|,\left|g_{2}\right|, \arg \left(g_{2}\right), \operatorname{Re} \tau$, and $\operatorname{Im} \tau$ to describe the 18 measured quantities, including the quark masses $m_{u, c, t}$ and $m_{d, s, b}$, the CKM mixing parameters $\theta_{12}^{q}$, $\theta_{13}^{q}, \theta_{23}^{q}$, and $\delta_{C P}^{q}$, the charged lepton masses $m_{e, \mu, \tau}$, the neutrino mass-squared splittings $\Delta m_{21}^{2}$ and $\Delta m_{31}^{2}$, and the lepton mixing angles $\theta_{12}^{l}, \theta_{13}^{l}$, and $\theta_{23}^{l}$. From Table VI, we see that the charged fermion masses and the CKM mixing matrix have been measured very precisely and their $1 \sigma$ ranges are quite narrow. Hence, the input parameters are also constrained to be in very narrow regions to accommodate the experimental data. We find that the regions of $\tau$ allowed by the experimental data on the quark and lepton sectors overlap, and excellent agreement with the measured values of the observables in Table VI can be achieved, as shown in Fig. 1. In order to make the common region of $\tau$ visible, we require that the quark mass ratios $y_{c} / y_{t}, y_{d} / y_{s}$, $y_{s} / y_{b}$ and the $C P$-violating phase $\delta_{C P}^{q}$ lie in the $2 \sigma$ intervals, the quark mixing angles $\theta_{12}^{q}, \theta_{13}^{q}, \theta_{23}^{q}$ and the charged lepton mass ratio $y_{e} / y_{\mu}, y_{\mu} / y_{\tau}$ lie in the $5 \sigma$ intervals, and the three neutrino mixing angles and neutrino mass-squared differences lie in their $3 \sigma$ ranges. It is notable that all of the quark and lepton mixing parameters are predicted to lie in narrow regions. In particular, the atmospheric mixing angle $\theta_{23}$ is in the second octant, and the lepton Dirac $C P$ phase $\delta_{C P}^{l}$ is determined to be around $3 \pi / 2$.

\section{NONMINIMAL KÄHLER POTENTIAL}

In the bottom-up models with modular symmetry, the finite modular symmetry is not very restrictive for the Kähler potential. It has been shown that the Kähler potential is not completely fixed by the modular symmetry [30], and the same holds true in discrete flavor symmetry models [80,81]. Besides the minimal Kähler potential chosen in Eq. (15), the Kähler potential can receive unsuppressed contributions from modular forms, and many other terms are also compatible with the modular symmetry. In the quark sector, the most general Kähler potential can be written as
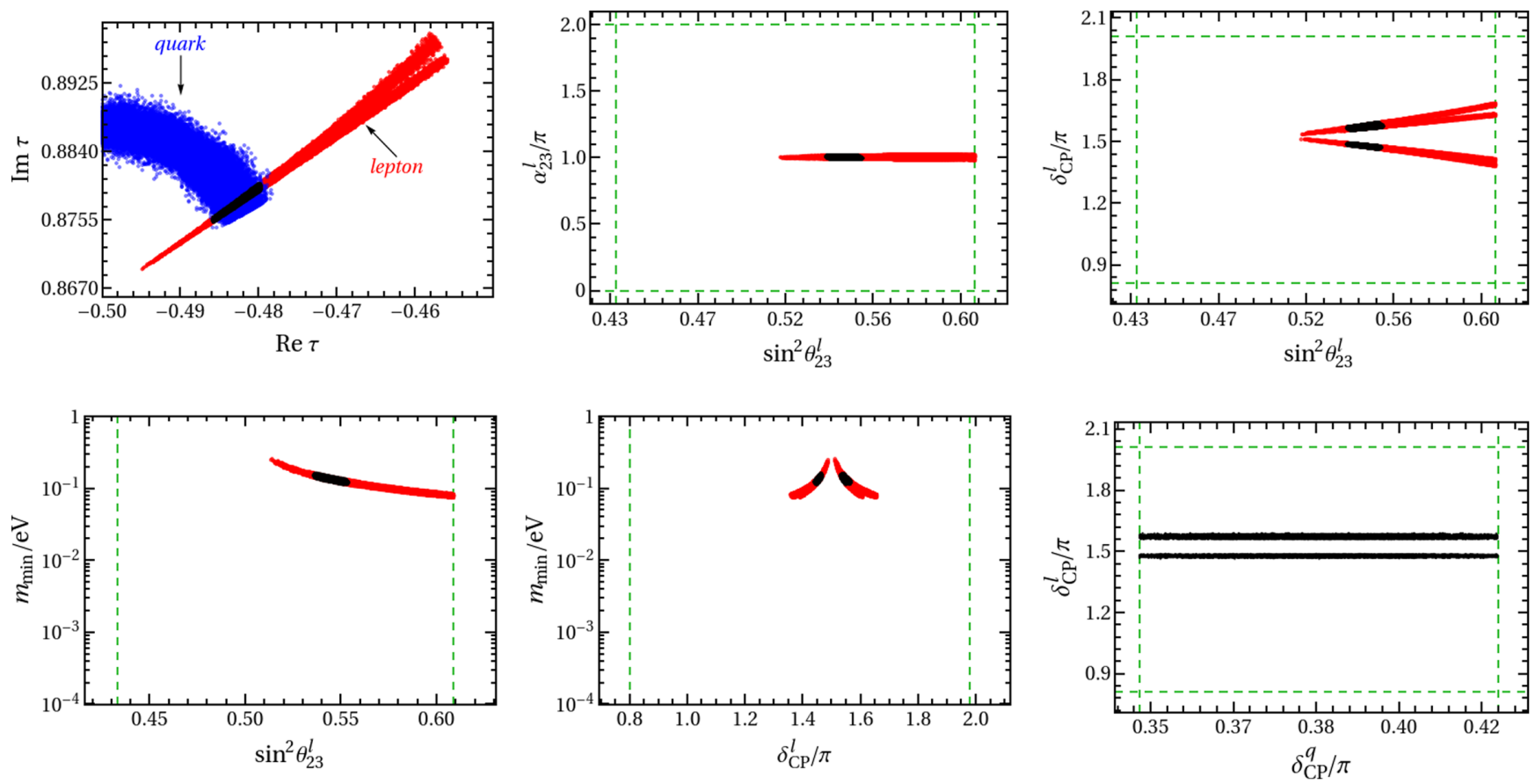

FIG. 1. The region of $\tau$ allowed by the experimental data in the $\operatorname{Re} \tau-\operatorname{Im} \tau$ plane and the correlation between different observables. The quark masses and mixing parameters can be obtained in the blue region, while the lepton masses and parameters can be obtained in the red region. The green dashed lines denote the experimentally preferred $3 \sigma$ ranges adapted from Ref. [1]. 


$$
\mathcal{K}=\mathcal{K}_{\min }+\Delta \mathcal{K}
$$

with

$$
\mathcal{K}_{\text {min }}=(-i \tau+i \bar{\tau})^{-k_{Q_{i}}} Q_{i}^{\dagger} Q_{i}+(-i \tau+i \bar{\tau})^{-k_{q_{i}^{c}}} q_{i}^{c \dagger} q_{i}^{c},
$$

and

$$
\begin{aligned}
\Delta \mathcal{K}= & \sum_{k_{1}, k_{2}, \mathbf{r}_{1}, \mathbf{r}_{2}} \lambda_{Q}^{i j}(-i \tau+i \bar{\tau})^{-k_{Q_{i}}+k_{1}}\left(Q_{i}^{\dagger} Y_{\mathbf{r}_{1}}^{\left(k_{1}\right) \dagger} Y_{\mathbf{r}_{2}}^{\left(k_{2}\right)} Q_{j}\right)_{\mathbf{1}} \\
& +\sum_{k_{3}, k_{4}, \mathbf{r}_{1}, \mathbf{r}_{2}} \lambda_{q^{c}}^{i j}(-i \tau+i \bar{\tau})^{-k_{q_{i}^{c}}+k_{3}}\left(q_{i}^{c \dagger} Y_{\mathbf{r}_{1}}^{\left(k_{3}\right) \dagger} Y_{\mathbf{r}_{2}}^{\left(k_{4}\right)} q_{j}^{c}\right)_{\mathbf{1}} \\
& + \text { H.c. }
\end{aligned}
$$

Note that there are generally a few independent contractions into $T^{\prime}$ singlets for each operator, and the modular weights should satisfy the following constraints:

$-k_{Q_{i}}+k_{1}=-k_{Q_{j}}+k_{2}, \quad-k_{q_{i}^{c}}+k_{3}=-k_{q_{j}^{c}}+k_{4}$.

The weight $k$ of the modular forms $Y_{\mathbf{r}}^{(k)}$ can run from 1 to $+\infty$, and thus an infinite number of possible terms could be present in the most general Kähler potential. The presence of free coefficients $\lambda_{Q}^{i j}$ and $\lambda_{q^{c}}^{i j}$ accompanying each operator in Eq. (71) could reduce the predictivity of modular invariance models, and the impacts of these additional terms on the predictions for both masses and mixing parameters are sizable, as shown in Ref. [30]. After the complex modulus $\tau$ acquires a vacuum expectation value $\langle\tau\rangle$, these additional terms $\Delta \mathcal{K}$ lead to departures from the canonical Kähler metric, i.e.,

$\mathcal{K}_{Q}^{i j}=\frac{\partial^{2} \mathcal{K}}{\partial Q_{i}^{\dagger} \partial Q_{j}}=-\langle i \tau+i \bar{\tau}\rangle^{-k_{Q_{i}}} \delta^{i j}+\Delta \mathcal{K}_{Q}^{i j}(\langle\tau\rangle)$,

$\mathcal{K}_{q^{c}}^{i j}=\frac{\partial^{2} \mathcal{K}}{\partial q_{i}^{c \dagger} \partial q_{j}^{c}}=-\langle i \tau+i \bar{\tau}\rangle^{-k_{q_{i}^{c}}^{c}} \delta^{i j}+\Delta \mathcal{K}_{q^{c}}^{i j}(\langle\tau\rangle)$.

Here both Kähler metric matrices $\mathcal{K}_{Q}$ and $\mathcal{K}_{q^{c}}$ are Hermitian and positive, and they can be rewritten as

$\mathcal{K}_{Q}=\Sigma_{Q} D_{Q}^{2} \Sigma_{Q}^{\dagger}, \quad \mathcal{K}_{u^{c}}=\Sigma_{u^{c}} D_{u^{c}}^{2} \Sigma_{u^{c}}^{\dagger}, \quad \mathcal{K}_{d^{c}}=\Sigma_{d^{c}} D_{d^{c}}^{2} \Sigma_{d^{c}}^{\dagger}$,

where $\Sigma_{Q, u^{c}, d^{c}}$ are unitary matrices and $D_{Q, u^{c}, d^{c}}$ are diagonal matrices with positive nonvanishing entries. In order to obtain a canonical Kähler metric $\mathcal{K}^{i j} \rightarrow \hat{\mathcal{K}}^{i j}=\delta^{i j}$ such that the quark fields are canonically normalized, we perform the field redefinitions

$$
\begin{aligned}
Q & =H_{Q} \hat{Q}, & H_{Q} & =\Sigma_{Q} D_{Q}^{-1} \Sigma_{Q}^{\dagger}, \\
u^{c} & =H_{u^{c}} \hat{u}^{c}, & & H_{u^{c}}=\Sigma_{u^{c}} D_{u^{c}}^{-1} \Sigma_{u^{c}}^{\dagger}, \\
d^{c} & =H_{d^{c}} \hat{d}^{c}, & H_{d^{c}} & =\Sigma_{d^{c}} D_{d^{c}}^{-1} \Sigma_{d^{c}}^{\dagger},
\end{aligned}
$$

where $H_{Q, u^{c}, d^{c}}$ are Hermitian. If $D_{Q, u^{c}, d^{c}}$ or $\Sigma_{Q, u^{c}, d^{c}}$ are proportional to the unit matrix, we see that one should rescale the quark fields to get canonical kinetic terms, and the effect of such rescaling can be absorbed into the arbitrary coefficients of the model. The field redefinition in Eq. (76) affects the superpotential or, equivalently, the Yukawa coupling matrices,

$$
\begin{aligned}
\mathcal{W} & =u^{c T} Y_{u} Q H_{u}+d^{c T} Y_{d} Q H_{d} \rightarrow \hat{\mathcal{W}} \\
& =\hat{u}^{c T} \hat{Y}_{u} \hat{Q} H_{u}+\hat{d}^{c T} \hat{Y}_{d} \hat{Q} H_{d},
\end{aligned}
$$

with

$$
\hat{Y}_{u}=H_{u^{c}}^{T} Y_{u} H_{Q}, \quad \hat{Y}_{d}=H_{d^{c}}^{T} Y_{d} H_{Q} .
$$

Diagonalizing the quark mass matrices in the basis of canonically normalized fields, we can obtain quark mass eigenvalues and the CKM matrix,

$\hat{U}_{u}^{\dagger} \hat{Y}_{u}^{\dagger} \hat{Y}_{u} \hat{U}_{u}=\operatorname{diag}\left(y_{u}^{2}, y_{c}^{2}, y_{t}^{2}\right)$,

$\hat{U}_{d}^{\dagger} \hat{Y}_{d}^{\dagger} \hat{Y}_{d} \hat{U}_{d}=\operatorname{diag}\left(y_{d}^{2}, y_{s}^{2}, y_{b}^{2}\right), \quad V_{\mathrm{CKM}}=\hat{U}_{u}^{\dagger} \hat{U}_{d}$.

Since $D_{Q, u, d}$ is generally not proportional to the unit matrix because of the nondiagonal terms $\Delta \mathcal{K}_{Q}^{i j}(\langle\tau\rangle)$ and $\Delta \mathcal{K}_{q^{c}}^{i j}(\langle\tau\rangle)$ in the Kähler metrics, the additional term $\Delta \mathcal{K}$ in the Kähler potential can lead to nontrivial effects on the observables, and it might significantly modify the predictions of a model [30,80,81].

In the present work, we have focused on doublet plus singlet assignment $Q_{D} \sim \mathbf{2}^{i}, Q_{3} \sim \mathbf{1}^{j}$ for the left-handed quarks. From Eq. (71), we know that the most general form of the extra Kähler potential is given by

$$
\begin{aligned}
\Delta \mathcal{K}_{Q}= & \sum_{k_{1}, k_{2}, k_{3}, \mathbf{r}_{1}, \mathbf{r}_{2}}(-i \tau+i \bar{\tau})^{-k_{Q_{D}}+k_{1}}\left(Q_{D}^{\dagger} Y_{\mathbf{r}_{1}}^{\left(k_{1}\right) \dagger} Q_{D} Y_{\mathbf{r}_{2}}^{\left(k_{1}\right)}\right)_{\mathbf{1}} \\
& +(-i \tau+i \bar{\tau})^{-k_{Q_{3}}+k_{1}} Q_{3}^{\dagger} Q_{3}\left(Y_{\mathbf{r}_{1}}^{\left(k_{1}\right) \dagger} Y_{\mathbf{r}_{2}}^{\left(k_{1}\right)}\right)_{1} \\
& +(-i \tau+i \bar{\tau})^{-k_{Q_{D}}+k_{2}}\left(Q_{D}^{\dagger} Y_{\mathbf{r}_{1}}^{\left(k_{2}\right) \dagger} Q_{3} Y_{\mathbf{r}_{2}}^{\left(k_{3}\right)}\right)_{\mathbf{1}}+\text { H.c., }
\end{aligned}
$$

where we suppress all couplings in front of each operator, and the modular weights fulfill $k_{2}-k_{3}=k_{Q_{D}}-k_{Q_{3}}$. In a similar fashion, if the right-handed quarks transform as a doublet and a singlet $q_{D}^{c} \sim \mathbf{2}^{k}, q_{3}^{c} \sim \mathbf{1}^{l}$ under $T^{\prime}$, the Kähler potential $\Delta \mathcal{K}_{q^{c}}$ has the following form: 


$$
\begin{aligned}
\Delta \mathcal{K}_{q^{c}}= & \sum_{k_{1}, k_{2}, k_{3}, \mathbf{r}_{1}, \mathbf{r}_{2}}(-i \tau+i \bar{\tau})^{-k_{q_{D}^{c}}^{c}+k_{1}}\left(q_{D}^{c \dagger} Y_{\mathbf{r}_{1}}^{\left(k_{1}\right) \dagger} q_{D}^{c} Y_{\mathbf{r}_{2}}^{\left(k_{1}\right)}\right)_{\mathbf{1}} \\
& +(-i \tau+i \bar{\tau})^{-k_{q_{3}^{c}}+k_{1}} q_{3}^{c \dagger} q_{3}^{c}\left(Y_{\mathbf{r}_{1}}^{\left(k_{1}\right) \dagger} Y_{\mathbf{r}_{2}}^{\left(k_{1}\right)}\right)_{\mathbf{1}} \\
& +(-i \tau+i \bar{\tau})^{-k_{q_{D}^{c}}+k_{2}}\left(q_{D}^{c \dagger} Y_{\mathbf{r}_{1}}^{\left(k_{2}\right) \dagger} q_{3}^{c} Y_{\mathbf{r}_{2}}^{\left(k_{3}\right)}\right)_{\mathbf{1}}+\text { H.c. },
\end{aligned}
$$

with $k_{2}-k_{3}=k_{q_{D}^{c}}-k_{q_{3}^{c}}$. For the singlet assignment $q_{a}^{c} \sim \mathbf{1}^{l_{a}}(a=1,2,3), \Delta \mathcal{K}_{q^{c}}$ reads as

$$
\begin{aligned}
\Delta \mathcal{K}_{q^{c}}= & \sum_{a, b, k_{1}, k_{2}, \mathbf{r}_{1}, \mathbf{r}_{2}}(-i \tau+i \bar{\tau})^{-k_{q_{a}^{c}}+k_{1}}\left(q_{a}^{c \dagger} Y_{\mathbf{r}_{1}}^{\left(k_{1}\right) \dagger} q_{b}^{c} Y_{\mathbf{r}_{2}}^{\left(k_{2}\right)}\right)_{1} \\
& + \text { H.c., }
\end{aligned}
$$

with $k_{1}-k_{2}=k_{q_{a}^{c}}-k_{q_{b}^{c}}$. Since the new parameters appearing in the Kähler potential considerably reduce the predictability of the approach [30], a better understanding of the Kähler potential is required. However, a way to better control the Kähler potential remains an open question. The Kähler potential would be better constrained in some more complete setting such as string theory [74]; nevertheless, the complete expressions for higher-order terms are still unknown. Recently, a new approach based on topdown model building in string theory was proposed $[25,29,75,76]$, namely, a hybrid of the top-down and bottom-up approaches to modular flavor symmetry [75]. This new approach combines the traditional flavor symmetry and the modular flavor symmetry, and introduces the concept of eclectic flavor groups. This new scheme severely restricts the representations and modular weights of matter fields, such that both the superpotential and Kähler potential are strongly constrained [76]. In order to show the impact of the additional terms in the Kähler potential, we give a concrete example in the following. The transformation properties of the quark fields under the $S U(2)_{L} \times U(1)_{Y}$ gauge group and $T^{\prime}$ modular symmetry are summarized in Table $X$. The modular-invariant superpotentials for up- and down-quark Yukawa couplings read as

$$
\begin{aligned}
& \mathcal{W}_{u}=y_{1}^{u} u^{c} Q_{D} Y_{\mathbf{2}^{\prime}}^{(5)} H_{u}+y_{2}^{u} c^{c} Q_{D} Y_{\mathbf{2}^{\prime \prime}}^{(3)} H_{u}+y_{3}^{u} t^{c} Q_{3} Y_{\mathbf{1}^{\prime}}^{(4)} H_{u} \\
& \mathcal{W}_{d}=y_{1}^{d} d_{D}^{c} Q_{D} Y_{\mathbf{3}}^{(4)} H_{d}+y_{2}^{d} d_{D}^{c} Q_{D} Y_{\mathbf{1}}^{(4)} H_{d}+y_{3}^{d} b^{c} Q_{3} H_{d},
\end{aligned}
$$

where the coupling constants $y_{1,2,3}^{u}$ and $y_{1,3}^{d}$ can be taken to be real and positive by rephasing the quark fields, while $y_{2}^{d}$ is generically a complex number. Applying the decomposition rules of the $T^{\prime}$ tensor products in Appendix A, we find that the quark mass matrices are given by

$$
\begin{aligned}
M_{u} & =\left(\begin{array}{ccc}
y_{1}^{u} Y_{\mathbf{2}^{\prime}, 2}^{(5)} & -y_{1}^{u} Y_{\mathbf{2}^{\prime}, 1}^{(5)} & 0 \\
y_{2}^{u} Y_{\mathbf{2}^{\prime \prime}, 2}^{(3)} & -y_{2}^{u} Y_{\mathbf{2}^{\prime \prime}, 1}^{(3)} & 0 \\
0 & 0 & y_{3}^{u}
\end{array}\right) v_{u}, \\
M_{d} & =\left(\begin{array}{ccc}
-\sqrt{2} e^{\frac{5 i \pi}{12}} y_{1}^{d} Y_{\mathbf{3}, 3}^{(4)} & y_{1}^{d} Y_{\mathbf{3}, 1}^{(4)}-y_{2}^{d} Y_{\mathbf{1}}^{(4)} & 0 \\
y_{1}^{d} Y_{\mathbf{3}, 1}^{(4)}+y_{2}^{d} Y_{\mathbf{1}}^{(4)} & -\sqrt{2} e^{\frac{7 i \pi}{12}} y_{1}^{d} Y_{\mathbf{3}, 2}^{(4)} & 0 \\
0 & 0 & y_{3}^{d}
\end{array}\right) v_{d} .
\end{aligned}
$$

We see that both the up-quark mass matrix $M_{u}$ and the down-quark mass matrix $M_{d}$ are block diagonal. As a result, only the Cabibbo angle can be accommodated, while the other two quark mixing angles $\theta_{13}^{q}, \theta_{23}^{q}$ and the $C P$ violation phase $\delta_{C P}^{q}$ vanish if only the superpotential is considered. Now we proceed to consider the contribution of the Kähler potential. With the transformation properties of quark fields in Table X, we can use Eqs. (80)-(82) and straightforwardly write down the most general Kähler potential $\mathcal{K}$. As an example, we only consider one extra term of the Kähler potential in the right-hand up quark sector,

$$
\Delta \mathcal{K}_{u^{c}}=\lambda_{u^{c}}(-i \tau+i \bar{\tau})^{-2} u^{c \dagger} Y_{1}^{(4) \dagger} t^{c}+\text { H.c. }
$$

where $\lambda_{u^{c}}$ is an arbitrary complex number. The minimal Kähler potential $\mathcal{K}_{\min }^{u^{c}}$ is

$$
\begin{aligned}
\mathcal{K}_{\min }^{u^{c}}= & (-i \tau+i \bar{\tau})^{-6}\left(u^{c \dagger} u^{c}\right)_{\mathbf{1}}+(-i \tau+i \bar{\tau})^{-4}\left(c^{c \dagger} c^{c}\right)_{\mathbf{1}} \\
& +(-i \tau+i \bar{\tau})^{-2}\left(t^{c \dagger} t^{c}\right)_{\mathbf{1}} .
\end{aligned}
$$

Then, we can find the Kähler metric $\mathcal{K}_{u^{c}}$ as follows:

TABLE X. The transformation properties of the quark fields under the standard model gauge group $S U(2)_{L} \times U(1)_{Y}$ and under $T^{\prime}$ modular symmetry for the example model in which the contribution of the nonminimal Kähler potential is considered, where $-k_{I}$ refers to the modular weights. The two Higgs doublets $H_{u, d}$ are invariant under $T^{\prime}$ and their modular weights vanish.

\begin{tabular}{lcccccccc}
\hline \hline & $Q_{D}$ & $Q_{3}$ & $u^{c}$ & $c^{c}$ & $t^{c}$ & $d_{D}^{c} \equiv\left(d^{c}, s^{c}\right)$ & $b^{c}$ \\
\cline { 2 - 8 }$S U(2)_{L} \times U(1)_{Y}$ & & $(2,1 / 6)$ & $(2,1 / 6)$ & $(1,-2 / 3)$ & $(1,-2 / 3)$ & $(1,-2 / 3)$ & $(1,1 / 3)$ & $(1,1 / 3)$ \\
\hline Model VI & $T^{\prime}$ & $\mathbf{2}$ & $\mathbf{1}^{\prime}$ & $\mathbf{1}^{\prime}$ & $\mathbf{1}$ & $\mathbf{1}^{\prime}$ & $\mathbf{2}^{\prime \prime}$ & $\mathbf{1}^{\prime \prime}$ \\
& $k_{I}$ & -1 & 2 & 6 & 4 & 2 & 5 & -2 \\
\hline \hline
\end{tabular}


TABLE XI. The best-fit values of the input parameters as well as quark and lepton mass ratios and flavor mixing parameters for Model VI and the quark-lepton unified model Model VI $+\mathcal{D}_{6}$.

\begin{tabular}{|c|c|c|c|c|c|c|c|c|c|}
\hline Model VI & Input & $\begin{array}{c}m_{u} / m_{c} \\
0.00203\end{array}$ & $\begin{array}{c}\operatorname{Re} \tau \\
-0.48188 \\
\left|y_{2}^{d} / y_{1}^{d}\right| \\
3.58111 \\
m_{c} / m_{t} \\
0.00282\end{array}$ & $\begin{array}{c}\operatorname{Im} \tau \\
0.87803 \\
\arg \left(y_{2}^{d} / y_{1}^{d}\right) / \pi \\
0.13777 \\
m_{d} / m_{s} \\
0.04983\end{array}$ & $\begin{array}{c}\left|\lambda_{u^{c}}\right| \\
146.59563 \\
y_{3}^{d} / y_{1}^{d} \\
1.98074 \\
m_{s} / m_{b} \\
0.01824\end{array}$ & $\begin{array}{c}\arg \left(\lambda_{u^{c}}\right) / \pi \\
0.91937 \\
y_{1}^{u} v_{u} / \mathrm{GeV} \\
0.57486 \\
\theta_{12}^{q} \\
0.22539\end{array}$ & $\begin{array}{c}y_{2}^{u} / y_{1}^{u} \\
6.96006 \\
y_{1}^{d} v_{d} / \mathrm{GeV} \\
0.48880 \\
\theta_{13}^{q} \\
0.00349\end{array}$ & $\begin{array}{c}\theta_{23}^{q} \\
0.03832\end{array}$ & $\begin{array}{c}\delta_{C P}^{q} /^{\circ} \\
69.55066\end{array}$ \\
\hline Model VI $+\mathcal{D}_{6}$ & $\begin{array}{l}\text { Input } \\
\text { Obs }\end{array}$ & & $\begin{array}{c}\beta / \alpha \\
0.37487 \\
m_{e} / m_{\mu} \\
0.00474 \\
\sin ^{2} \theta_{12}^{l} \\
0.31012\end{array}$ & $\begin{array}{c}\gamma / \alpha \\
0.00433 \\
m_{\mu} / m_{\tau} \\
0.05858 \\
\sin ^{2} \theta_{13}^{l} \\
0.02222\end{array}$ & $\begin{array}{c}\left|g_{2} / g_{1}\right| \\
1.11456 \\
m_{1} / \mathrm{eV} \\
0.13012 \\
\sin ^{2} \theta_{23}^{l} \\
0.54786\end{array}$ & $\begin{array}{c}\arg \left(g_{2} / g_{1}\right) / \pi \\
1.62791 \\
m_{2} / \mathrm{eV} \\
0.13040 \\
\delta_{C P}^{l} /^{\circ} \\
278.10936\end{array}$ & $\begin{array}{c}\alpha v_{d} / \mathrm{GeV} \\
8.34809 \\
m_{3} / \mathrm{eV} \\
0.13948 \\
\alpha_{21} /^{\circ} \\
359.47123\end{array}$ & $\begin{array}{c}\left(g_{1}^{2} v_{u}^{2} / \Lambda\right) / \mathrm{eV} \\
0.41822 \\
\left|m_{e e}\right| / \mathrm{eV} \\
0.13028 \\
\alpha_{31} /^{\circ} \\
179.07277\end{array}$ & \\
\hline
\end{tabular}

$$
\begin{aligned}
\mathcal{K}_{u^{c}}= & \left(\begin{array}{ccc}
\langle-i \tau+i \bar{\tau}\rangle^{-6} & 0 & 0 \\
0 & \langle-i \tau+i \bar{\tau}\rangle^{-4} & 0 \\
0 & 0 & \langle-i \tau+i \bar{\tau}\rangle^{-2}
\end{array}\right) \\
& +\langle-i \tau+i \bar{\tau}\rangle^{-2}\left(\begin{array}{ccc}
0 & 0 & \lambda_{u^{c}} Y_{1}^{(4) \dagger} \\
0 & 0 & 0 \\
\lambda_{u^{c}}^{*} Y_{1}^{(4)} & 0 & 0
\end{array}\right)+\ldots
\end{aligned}
$$

We can rescale the up-type quark superfields $u^{c} \rightarrow$ $\langle-i \tau+i \bar{\tau}\rangle^{3} u^{c}, c^{c} \rightarrow\langle-i \tau+i \bar{\tau}\rangle^{2} c^{c}$, and $t^{c} \rightarrow\langle-i \tau+i \bar{\tau}\rangle t^{c}$ which can be absorbed into the arbitrary coefficients in this model. Then, the Kähler metric $\mathcal{K}_{u^{c}}$ simplifies to

$$
\begin{aligned}
\mathcal{K}_{u^{c}}= & \left(\begin{array}{lll}
1 & 0 & 0 \\
0 & 1 & 0 \\
0 & 0 & 1
\end{array}\right) \\
& +\langle-i \tau+i \bar{\tau}\rangle^{2}\left(\begin{array}{ccc}
0 & 0 & \lambda_{u^{c}} Y_{1}^{(4) \dagger} \\
0 & 0 & 0 \\
\lambda_{u^{c}}^{*} Y_{1}^{(4)} & 0 & 0
\end{array}\right)+\cdots
\end{aligned}
$$

The Kähler metric is not diagonal anymore because of the additional term in Eq. (85) so that the third and first two generations can mix. A numerical analysis is performed, and we obtain the correct values of the quark masses, quark mixing angles, and $C P$ violation phase. The numerical fitting results are shown in Table XI. In a similar fashion as in Sec. VI, this model together with the lepton model $\mathcal{D}_{6}$ can give a unified interpretation of quark and lepton masses and flavor mixing for certain values of the input parameters, as shown in Table XI. The nonminimal Kähler potential in the lepton sector is discussed in Appendix C.

\section{CONCLUSION AND SUMMARY}

Modular invariance as the flavor symmetry is a promising approach to understand the puzzle of quark and lepton masses and flavor mixing. The Yukawa couplings are modular forms of a certain level $N$ in this approach. We have generalized this formalism to modular forms of general integer weights which can be arranged into irreducible representations of the homogeneous finite modular group $\Gamma_{N}^{\prime}$ [61]. Notice that $\Gamma_{N}^{\prime}$ is the double covering of $\Gamma_{N}$. It is well known that $S U(2)$ is the double covering group of $S O(3)$, and two $S U(2)$ elements correspond to one $S O(3)$ element with the same Euler angles. $\Gamma_{N}^{\prime}$ can be regarded as the inverse image of the inhomogeneous finite modular group $\Gamma_{N}$ under this map for smaller $N$. In addition to the irreducible representations of $\Gamma_{N}, \Gamma_{N}^{\prime}$ has other new representations [61]. The even-weight modular forms of level $N$ transform in the irreducible representations of $\Gamma_{N}$ [11], while the odd-weight modular forms of level $N$ are arranged into the new representations of $\Gamma_{N}^{\prime}$ [61]. The oddweight modular forms provide us with an interesting opportunity for fermion mass model building.

The ansatz of texture zeros has been widely studied to reduce the number of free parameters. It has been shown that the texture zeros of the fermion mass matrices can be enforced by means of Abelian symmetries. In the present work, we showed that the texture zeros of the fermion mass matrices can be naturally produced if we properly assign the representations and weights of the matter fields under the modular symmetry. As a concrete example, we imposed the $\Gamma_{3}^{\prime} \cong T^{\prime}$ modular symmetry on the quark sector. We assigned the first two generations of left-handed quark doublets to a $T^{\prime}$ doublet, and the third generation of left-handed quarks is a singlet of $T^{\prime}$. As regards the right-handed quark fields, we considered two scenarios where the three generations of right-handed quarks either transform as a doublet and a singlet under $T^{\prime}$ or are three singlets of $T^{\prime}$. We found that the quark mass matrix can take six possible forms with zero entries up to row and column permutations: Case $\mathcal{A}$, Case $\mathcal{B}$, Case $\mathcal{C}$, Case $\mathcal{D}$, Case $\mathcal{E}$, and Case $\mathcal{F}$, given in Eqs. (40), (52), and (53). Combining the up- and down-quark sectors, we can obtain the possible up-quark and down-quark mass matrices predicted by $T^{\prime}$ modular symmetry. It is usually assumed that the mass matrix is symmetric or Hermitian in 
texture-zero models. In our approach, the explicit form of the mass matrix is completely fixed by modular symmetry and it is generally neither symmetric nor Hermitian. We presented five interesting quark models, summarized in Table V. These models contain only 10 or 11 independent real parameters including the complex modulus $\tau$, and the experimental data on quark masses and the CKM mixing matrix can be accommodated very well.

Furthermore, we investigated the lepton sector with the $T^{\prime}$ modular symmetry. The lepton fields were assumed to transform as a triplet 3 or singlets $\mathbf{1}, \mathbf{1}^{\prime}, \mathbf{1}^{\prime \prime}$ under $T^{\prime}$ such that the $T^{\prime}$ modular symmetry cannot be distinguished from the $A_{4}$ modular symmetry in the lepton sector since doublet representations of $T^{\prime}$ are not involved. A systematic classification of lepton models with $A_{4}$ modular symmetry was performed in Ref. [43]. We combined the five quark models in Table $\mathrm{V}$ with the $\mathcal{D}_{6}$ and $\mathcal{D}_{9}$ lepton models of Ref. [43] to give a unified description of both quark and lepton masses and flavor mixing. It is highly nontrivial that the resulting models can produce very good fits to the experimental data despite containing a fewer number of free parameters than the observables.

In summary, the modular symmetry can naturally produce texture zeros in the fermion mass matrix if odd-weight modular forms are considered. The modular invariance approach has the merits of both Abelian flavor symmetry and discrete non-Abelian flavor symmetry. It would be interesting to investigate the possible texture-zero structures of the lepton sector that can be obtained from modular symmetry.

In the present paper, we followed the bottom-up model construction approach of modular symmetry to describe the flavor structure of the SM. It is interesting to investigate whether the benchmark quark-lepton unification models in Sec. VI can be embedded in a string theory framework. It has been shown that the finite modular group $T^{\prime}$ can naturally appear in simple string constructions such as $Z_{3}$ orbifolds [25,29]. Therefore, we expect that certain (but not all) models of the present paper or at the least texture-zero structure of quark mass matrix could be realized in the lowenergy effective theory, although explicit model building in string theory is needed. The phenomenological bottom-up models based on modular invariance can explain many different aspects of the flavor puzzle in the SM. However, the possible connections between this approach and a more fundamental theory such as string theory are still not clear; see Refs. $[25,29,75]$ for recent progress in this direction. Discrete flavor symmetries and modular invariance could appear simultaneously in top-down model building in string theory. Recently, it was suggested to extend the traditional discrete flavor group by finite modular symmetries [76]. The Kähler potential and superpotential are severely constrained in this new scheme; in particular, the off-diagonal contributions to the Kähler metric are forbidden. As a result, the modular form-dependent terms in the Kähler potential do not considerably change the phenomenological predictions obtained by assuming a minimal Kähler potential. Hence, it is a reasonable and good approximation to take the standard minimal Kähler potential in bottom-up model construction.

\section{ACKNOWLEDGMENTS}

This work is supported by the National Natural Science Foundation of China under Grant Nos 11975224 and 11835013.

\section{APPENDIX A: GROUP THEORY OF $T^{\prime}$}

The $T^{\prime}$ group is the double covering of the tetrahedral group $A_{4}$. All of the elements of $T^{\prime}$ can be generated by three generators $S, T$, and $\mathbb{R}$, which obey the following relations:

$$
S^{2}=\mathbb{R}, \quad(S T)^{3}=T^{3}=\mathbb{R}^{2}=1, \quad \mathbb{R} T=T \mathbb{R} .
$$

Hence, the generator $\mathbb{R}$ commutes with all elements of the group. Besides the $A_{4}$ representations-one triplet 4 and three singlets $\mathbf{1}, \mathbf{1}^{\prime}$, and $\mathbf{1}^{\prime \prime}$ - the $T^{\prime}$ group has three twodimensional irreducible representations $\mathbf{2}, \mathbf{2}^{\prime}$, and $\mathbf{2}^{\prime \prime}$. In the present work, we adopt the same basis as in Ref. [61], in particular using the following explicit representation matrices for the generators $S, T$, and $\mathbb{R}$ in different irreps:

$$
\begin{aligned}
& \text { 1: } S=1, \quad T=1, \quad \mathbb{R}=1 \text {, } \\
& \mathbf{1}^{\prime}: S=1, \quad T=\omega, \quad \mathbb{R}=1, \\
& \mathbf{1}^{\prime \prime}: S=1, \quad T=\omega^{2}, \quad \mathbb{R}=1, \\
& \text { 2: } S=-\frac{1}{\sqrt{3}}\left(\begin{array}{cc}
i & \sqrt{2} e^{i \pi / 12} \\
-\sqrt{2} e^{-i \pi / 12} & -i
\end{array}\right) \text {, } \\
& T=\left(\begin{array}{cc}
\omega & 0 \\
0 & 1
\end{array}\right), \quad \mathbb{R}=-\left(\begin{array}{ll}
1 & 0 \\
0 & 1
\end{array}\right), \\
& \mathbf{2}^{\prime}: S=-\frac{1}{\sqrt{3}}\left(\begin{array}{cc}
i & \sqrt{2} e^{i \pi / 12} \\
-\sqrt{2} e^{-i \pi / 12} & -i
\end{array}\right) \text {, } \\
& T=\left(\begin{array}{cc}
\omega^{2} & 0 \\
0 & \omega
\end{array}\right), \quad \mathbb{R}=-\left(\begin{array}{cc}
1 & 0 \\
0 & 1
\end{array}\right), \\
& \mathbf{2}^{\prime \prime}: S=-\frac{1}{\sqrt{3}}\left(\begin{array}{cc}
i & \sqrt{2} e^{i \pi / 12} \\
-\sqrt{2} e^{-i \pi / 12} & -i
\end{array}\right) \text {, } \\
& T=\left(\begin{array}{cc}
1 & 0 \\
0 & \omega^{2}
\end{array}\right), \quad \mathbb{R}=-\left(\begin{array}{cc}
1 & 0 \\
0 & 1
\end{array}\right), \\
& \text { 3: } S=\frac{1}{3}\left(\begin{array}{ccc}
-1 & 2 & 2 \\
2 & -1 & 2 \\
2 & 2 & -1
\end{array}\right), \quad T=\left(\begin{array}{ccc}
1 & 0 & 0 \\
0 & \omega & 0 \\
0 & 0 & \omega^{2}
\end{array}\right) \text {, } \\
& \mathbb{R}=\left(\begin{array}{lll}
1 & 0 & 0 \\
0 & 1 & 0 \\
0 & 0 & 1
\end{array}\right)
\end{aligned}
$$


with $\omega=e^{i 2 \pi / 3}$. The generator $\mathbb{R}$ is represented by an identity matrix for the odd-dimensional representations $\mathbf{1}, \mathbf{1}^{\prime}$, $\mathbf{1}^{\prime \prime}$, and 3 . Therefore, the elements of $T^{\prime}$ coincide two by two and can be described by the same matrices that represent the elements in $A_{4}$ for these representations. The Kronecker products between different irreducible representations of $T^{\prime}$ are given by

$\mathbf{1}^{a} \otimes \mathbf{r}^{b}=\mathbf{r}^{b} \otimes \mathbf{1}^{a}=\mathbf{r}^{a+b(\bmod 3)}, \quad$ for $\mathbf{r}=\mathbf{1}, \mathbf{2}$,

$\mathbf{1}^{a} \otimes \mathbf{3}=\mathbf{3} \otimes \mathbf{1}^{a}=\mathbf{3}$,

$\mathbf{2}^{a} \otimes \mathbf{2}^{b}=\mathbf{3} \oplus \mathbf{1}^{a+b+1(\bmod 3)}$,

$2^{a} \otimes 3=3 \otimes 2^{a}=2 \oplus 2^{\prime} \oplus 2^{\prime \prime}$,

$\mathbf{3} \otimes \mathbf{3}=\mathbf{3}_{S} \oplus \mathbf{3}_{A} \oplus \mathbf{1} \oplus \mathbf{1}^{\prime} \oplus \mathbf{1}^{\prime \prime}$,

where $a, b=0,1,2$ and we have denoted $\mathbf{1} \equiv \mathbf{1}^{0}, \mathbf{1}^{\prime} \equiv \mathbf{1}^{1}$, $\mathbf{1}^{\prime \prime} \equiv \mathbf{1}^{2}$ for singlet representations and $\mathbf{2} \equiv \mathbf{2}^{0}, \mathbf{2}^{\prime} \equiv \mathbf{2}^{1}$, $2^{\prime \prime} \equiv \mathbf{2}^{2}$ for the doublet representations. The notations $\mathbf{3}_{S}$ and $\mathbf{3}_{A}$ stand for the symmetric and antisymmetric triplet combinations, respectively. The CG coefficients in our working basis can be found in Ref. [61], but we list them in the following for completeness. We use $\alpha_{i}$ to denote the elements of the first representation, and $\beta_{i}$ to indicate those of the second representation of the product:

$$
\begin{gathered}
\mathbf{1}^{a} \otimes \mathbf{1}^{b}=\mathbf{1}^{a+b(\bmod 3)} \sim \alpha \beta, \\
\mathbf{1}^{a} \otimes \mathbf{2}^{b}=\mathbf{2}^{a+b(\bmod 3)} \sim\left(\begin{array}{c}
\alpha \beta_{1} \\
\alpha \beta_{2}
\end{array}\right), \\
\mathbf{1}^{\prime} \otimes \mathbf{3}=\mathbf{3} \sim\left(\begin{array}{c}
\alpha \beta_{3} \\
\alpha \beta_{1} \\
\alpha \beta_{2}
\end{array}\right), \\
\mathbf{1}^{\prime \prime} \otimes \mathbf{3}=\mathbf{3} \sim\left(\begin{array}{c}
\alpha \beta_{2} \\
\alpha \beta_{3} \\
\alpha \beta_{1}
\end{array}\right) .
\end{gathered}
$$

$2 \otimes 2=2^{\prime} \otimes 2^{\prime \prime}=3 \oplus 1^{\prime}$

$$
\text { with }\left\{\begin{array}{c}
\mathbf{1}^{\prime} \sim \alpha_{1} \beta_{2}-\alpha_{2} \beta_{1}, \\
\mathbf{3} \sim\left(\begin{array}{c}
e^{i \pi / 6} \alpha_{2} \beta_{2} \\
\frac{1}{\sqrt{2}} e^{i 7 \pi / 12}\left(\alpha_{1} \beta_{2}+\alpha_{2} \beta_{1}\right) \\
\alpha_{1} \beta_{1}
\end{array}\right),
\end{array}\right.
$$

$2 \otimes 2^{\prime}=2^{\prime \prime} \otimes 2^{\prime \prime}=3 \oplus 1^{\prime \prime}$

$$
\text { with }\left\{\begin{array}{l}
\mathbf{1}^{\prime \prime} \sim \alpha_{1} \beta_{2}-\alpha_{2} \beta_{1}, \\
\alpha_{1} \beta_{1} \\
\mathbf{3} \sim\left(\begin{array}{c}
e^{i \pi / 6} \alpha_{2} \beta_{2} \\
\frac{1}{\sqrt{2}} e^{i 7 \pi / 12}\left(\alpha_{1} \beta_{2}+\alpha_{2} \beta_{1}\right)
\end{array}\right),
\end{array}\right.
$$

$2 \otimes 2^{\prime \prime}=2^{\prime} \otimes 2^{\prime}=3 \oplus 1$

$$
\text { with }\left\{\begin{array}{c}
\mathbf{1} \sim \alpha_{1} \beta_{2}-\alpha_{2} \beta_{1}, \\
\mathbf{3} \sim\left(\begin{array}{c}
\frac{1}{\sqrt{2}} e^{i 7 \pi / 12}\left(\alpha_{1} \beta_{2}+\alpha_{2} \beta_{1}\right) \\
\alpha_{1} \beta_{1} \\
e^{i \pi / 6} \alpha_{2} \beta_{2}
\end{array}\right),
\end{array}\right.
$$

$2 \otimes 3=2 \oplus 2^{\prime} \oplus 2^{\prime \prime}$

$$
\text { with }\left\{\begin{array}{l}
\mathbf{2} \sim\left(\begin{array}{c}
\alpha_{1} \beta_{1}-\sqrt{2} e^{i 7 \pi / 12} \alpha_{2} \beta_{2} \\
-\alpha_{2} \beta_{1}+\sqrt{2} e^{i 5 \pi / 12} \alpha_{1} \beta_{3}
\end{array}\right), \\
\mathbf{2}^{\prime} \sim\left(\begin{array}{c}
\alpha_{1} \beta_{2}-\sqrt{2} e^{i 7 \pi / 12} \alpha_{2} \beta_{3} \\
-\alpha_{2} \beta_{2}+\sqrt{2} e^{i 5 \pi / 12} \alpha_{1} \beta_{1}
\end{array}\right), \\
\mathbf{2}^{\prime \prime} \sim\left(\begin{array}{c}
\alpha_{1} \beta_{3}-\sqrt{2} e^{i 7 \pi / 12} \alpha_{2} \beta_{1} \\
-\alpha_{2} \beta_{3}+\sqrt{2} e^{i 5 \pi / 12} \alpha_{1} \beta_{2}
\end{array}\right),
\end{array}\right.
$$

$\mathbf{2}^{\prime} \otimes \mathbf{3}=\mathbf{2} \oplus \mathbf{2}^{\prime} \oplus \mathbf{2}^{\prime \prime}$

$$
\text { with }\left\{\begin{aligned}
& \mathbf{2} \sim\left(\begin{array}{c}
\alpha_{1} \beta_{3}-\sqrt{2} e^{i 7 \pi / 12} \alpha_{2} \beta_{1} \\
-\alpha_{2} \beta_{3}+\sqrt{2} e^{i 5 \pi / 12} \alpha_{1} \beta_{2}
\end{array}\right), \\
& \mathbf{2}^{\prime} \sim\left(\begin{array}{c}
\alpha_{1} \beta_{1}-\sqrt{2} e^{i 7 \pi / 12} \alpha_{2} \beta_{2} \\
-\alpha_{2} \beta_{1}+\sqrt{2} e^{i 5 \pi / 12} \alpha_{1} \beta_{3}
\end{array}\right), \\
& \mathbf{2}^{\prime \prime} \sim\left(\begin{array}{c}
\alpha_{1} \beta_{2}-\sqrt{2} e^{i 7 \pi / 12} \alpha_{2} \beta_{3} \\
-\alpha_{2} \beta_{2}+\sqrt{2} e^{i 5 \pi / 12} \alpha_{1} \beta_{1}
\end{array}\right),
\end{aligned}\right.
$$

$2^{\prime \prime} \otimes 3=2 \oplus 2^{\prime} \oplus 2^{\prime \prime}$

$$
\text { with }\left\{\begin{array}{l}
\mathbf{2} \sim\left(\begin{array}{c}
\alpha_{1} \beta_{2}-\sqrt{2} e^{i 7 \pi / 12} \alpha_{2} \beta_{3} \\
-\alpha_{2} \beta_{2}+\sqrt{2} e^{i 5 \pi / 12} \alpha_{1} \beta_{1}
\end{array}\right), \\
\mathbf{2}^{\prime} \sim\left(\begin{array}{c}
\alpha_{1} \beta_{3}-\sqrt{2} e^{i 7 \pi / 12} \alpha_{2} \beta_{1} \\
-\alpha_{2} \beta_{3}+\sqrt{2} e^{i 5 \pi / 12} \alpha_{1} \beta_{2}
\end{array}\right), \\
\mathbf{2}^{\prime \prime} \sim\left(\begin{array}{c}
\alpha_{1} \beta_{1}-\sqrt{2} e^{i 7 \pi / 12} \alpha_{2} \beta_{2} \\
-\alpha_{2} \beta_{1}+\sqrt{2} e^{i 5 \pi / 12} \alpha_{1} \beta_{3}
\end{array}\right),
\end{array}\right.
$$

$\mathbf{3} \otimes \mathbf{3}=\mathbf{3}_{S} \oplus \mathbf{3}_{A} \oplus \mathbf{1} \oplus \mathbf{1}^{\prime} \oplus \mathbf{1}^{\prime \prime} \quad$ with

$$
\left\{\begin{aligned}
\mathbf{3}_{S} \sim\left(\begin{array}{l}
2 \alpha_{1} \beta_{1}-\alpha_{2} \beta_{3}-\alpha_{3} \beta_{2} \\
2 \alpha_{3} \beta_{3}-\alpha_{1} \beta_{2}-\alpha_{2} \beta_{1} \\
2 \alpha_{2} \beta_{2}-\alpha_{1} \beta_{3}-\alpha_{3} \beta_{1}
\end{array}\right), \\
\mathbf{3}_{A} \sim\left(\begin{array}{l}
\alpha_{2} \beta_{3}-\alpha_{3} \beta_{2} \\
\alpha_{1} \beta_{2}-\alpha_{2} \beta_{1} \\
\alpha_{3} \beta_{1}-\alpha_{1} \beta_{3}
\end{array}\right) \\
\mathbf{1} \sim \alpha_{1} \beta_{1}+\alpha_{2} \beta_{3}+\alpha_{3} \beta_{2} \\
\mathbf{1}^{\prime} \sim \alpha_{3} \beta_{3}+\alpha_{1} \beta_{2}+\alpha_{2} \beta_{1} \\
\mathbf{1}^{\prime \prime} \sim \alpha_{2} \beta_{2}+\alpha_{1} \beta_{3}+\alpha_{3} \beta_{1}
\end{aligned}\right.
$$




\section{APPENDIX B: HIGHER-WEIGHT MODULAR FORMS}

Using the contraction rules of the $T^{\prime}$ group in Appendix A, we can construct the modular forms of weight $k=5,6,7,8$ and level 3. The linear space of modular forms of weight $k$ and level 3 has dimension $k+1$. The action of the homogeneous finite modular group $T^{\prime}$ divides the space of weight- 5 modular forms into three doublets transforming the irreducible representations $\mathbf{2}, \mathbf{2}^{\prime}$, and $\mathbf{2}^{\prime \prime}$ of $T^{\prime}$. They are given by

$$
\begin{aligned}
& Y_{\mathbf{2}}^{(5)} \equiv\left(\begin{array}{c}
Y_{\mathbf{2}, 1}^{(5)} \\
Y_{\mathbf{2}, 2}^{(5)}
\end{array}\right)=\left(\begin{array}{c}
2 \sqrt{2} e^{i 7 \pi / 12} Y_{1}^{4} Y_{2}+e^{i \pi / 3} Y_{1} Y_{2}^{4} \\
2 \sqrt{2} e^{i 7 \pi / 12} Y_{1}^{3} Y_{2}^{2}+e^{i \pi / 3} Y_{2}^{5}
\end{array}\right), \\
& Y_{\mathbf{2}^{\prime}}^{(5)} \equiv\left(\begin{array}{c}
Y_{\mathbf{2}^{\prime}, 1}^{(5)} \\
Y_{\mathbf{2}^{\prime}, 2}^{(5)}
\end{array}\right)=\left(\begin{array}{c}
-Y_{1}^{5}+2(1-i) Y_{1}^{2} Y_{2}^{3} \\
-Y_{1}^{4} Y_{2}+2(1-i) Y_{1} Y_{2}^{4}
\end{array}\right), \\
& Y_{\mathbf{2}^{\prime \prime}}^{(5)} \equiv\left(\begin{array}{c}
Y_{\mathbf{2}^{\prime \prime}, 1}^{(5)} \\
Y_{\mathbf{2}^{\prime \prime}, 2}^{(5)}
\end{array}\right)=\left(\begin{array}{c}
5 e^{i \pi / 6} Y_{1}^{3} Y_{2}^{2}-(1-i) e^{i \pi / 6} Y_{2}^{5} \\
-\sqrt{2} e^{i 5 \pi / 12} Y_{1}^{5}-5 e^{i \pi / 6} Y_{1}^{2} Y_{2}^{3}
\end{array}\right) .
\end{aligned}
$$

The weight 6 modular forms can be decomposed into two triplets 3 and one singlet 1 of $T^{\prime}$, and they explicitly read

$$
\begin{aligned}
& Y_{1}^{(6)}=(1-i) e^{i \pi / 6} Y_{2}^{6}-(1+i) e^{i \pi / 6} Y_{1}^{6}-10 e^{i \pi / 6} Y_{1}^{3} Y_{2}^{3}, \\
& Y_{3 I}^{(6)} \equiv\left(\begin{array}{c}
Y_{3 I, 1}^{(6)} \\
Y_{3 I, 2}^{(6)} \\
Y_{3 I, 3}^{(6)}
\end{array}\right)=\left(\begin{array}{c}
-2(1-i) Y_{1}^{3} Y_{2}^{3}+i Y_{2}^{6} \\
-4 e^{i \pi / 6} Y_{1}^{4} Y_{2}^{2}-(1-i) e^{i \pi / 6} Y_{1} Y_{2}^{5} \\
2 \sqrt{2} e^{i 7 \pi / 12} Y_{1}^{5} Y_{2}+e^{i \pi / 3} Y_{1}^{2} Y_{2}^{4}
\end{array}\right), \\
& Y_{3 I I}^{(6)} \equiv\left(\begin{array}{c}
Y_{3 I I, 1}^{(6)} \\
Y_{3 I I, 2}^{(6)} \\
Y_{3 I I, 3}^{(6)}
\end{array}\right)=\left(\begin{array}{c}
-Y_{1}^{6}+2(1-i) Y_{1}^{3} Y_{2}^{3} \\
-e^{i \pi / 6} Y_{1}^{4} Y_{2}^{2}+2(1-i) e^{i \pi / 6} Y_{1} Y_{2}^{5} \\
4 e^{i \pi / 3} Y_{1}^{2} Y_{2}^{4}-(1+i) e^{i \pi / 3} Y_{1}^{5} Y_{2}
\end{array}\right) .
\end{aligned}
$$

For the modular forms of weight 7 , we have

$$
\begin{aligned}
& Y_{2 I}^{(7)} \equiv\left(\begin{array}{c}
Y_{2 I, 1}^{(7)} \\
Y_{2 I, 2}^{(7)}
\end{array}\right)=\left(\begin{array}{c}
3 i Y_{1} Y_{2}^{3}\left[2(1+i) Y_{1}^{3}+Y_{2}^{3}\right] \\
-4 Y_{1}^{6} Y_{2}+(1-i) Y_{1}^{3} Y_{2}^{4}-i Y_{2}^{7}
\end{array}\right), \\
& Y_{2 I I}^{(7)} \equiv\left(\begin{array}{c}
Y_{2 I I, 1}^{(7)} \\
Y_{2 I I, 2}^{(7)}
\end{array}\right)=\left(\begin{array}{c}
-Y_{1}\left[Y_{1}^{6}-(1-i) Y_{1}^{3} Y_{2}^{3}+4 i Y_{2}^{6}\right] \\
3 Y_{1}^{3} Y_{2}\left[Y_{1}^{3}-2(1-i) Y_{2}^{3}\right]
\end{array}\right), \\
& Y_{\mathbf{2}^{\prime}}^{(7)} \equiv\left(\begin{array}{c}
Y_{\mathbf{2}^{\prime}, 1}^{(7)} \\
Y_{\mathbf{2}^{\prime}, 2}^{(7)}
\end{array}\right)=\left(\begin{array}{c}
-3 e^{i \pi / 6} Y_{1}^{2} Y_{2}^{2}\left[Y_{1}^{3}-2(1-i) Y_{2}^{3}\right] \\
-e^{i \pi / 6} Y_{1}\left[Y_{1}^{3}-2(1-i) Y_{2}^{3}\right]\left[(1+i) Y_{1}^{3}-Y_{2}^{3}\right]
\end{array}\right), \\
& Y_{\mathbf{2}^{\prime \prime}}^{(7)} \equiv\left(\begin{array}{c}
Y_{\mathbf{2}^{\prime \prime}, 1}^{(7)} \\
Y_{\mathbf{2}^{\prime \prime}, 2}^{(7)}
\end{array}\right)=\left(\begin{array}{c}
{\left[2(1+i) Y_{1}^{3}+Y_{2}^{3}\right]\left(e^{i \pi / 3} Y_{1}^{3} Y_{2}+e^{i \pi / 12} \sqrt{2} Y_{2}^{4}\right)} \\
-3 e^{i \pi / 3} Y_{1}^{2} Y_{2}^{2}\left[2(1+i) Y_{1}^{3}+Y_{2}^{3}\right]
\end{array}\right) .
\end{aligned}
$$

Finally, the expressions of the weight- 8 modular forms of level 3 are as follows:

$$
\begin{aligned}
Y_{\mathbf{1}}^{(8)} & =\left[2(1+i) Y_{1}^{3}+Y_{2}^{3}\right]\left(4 e^{i \pi / 3} Y_{1}^{3} Y_{2}^{2}+e^{i \pi / 12} \sqrt{2} Y_{2}^{5}\right), \\
Y_{\mathbf{1}^{\prime}}^{(8)} & =4 Y_{1}^{7} Y_{2}-7(1-i) Y_{1}^{4} Y_{2}^{4}+4 i Y_{1} Y_{2}^{7}, \\
Y_{\mathbf{1}^{\prime \prime}}^{(8)} & =\left[Y_{1}^{4}-2(1-i) Y_{1} Y_{2}^{3}\right]^{2}, \\
Y_{\mathbf{3} I}^{(8)} & \equiv\left(\begin{array}{c}
Y_{3 I, 1}^{(8)} \\
Y_{\mathbf{3} I, 2}^{(8)} \\
Y_{\mathbf{3} I, 3}^{(8)}
\end{array}\right)=\left(\begin{array}{c}
-e^{i 2 \pi / 3} Y_{2}\left[-4 i Y_{1}^{6} Y_{2}+(1+i) Y_{1}^{3} Y_{2}^{4}+Y_{2}^{7}\right] \\
e^{i 5 \pi / 6}\left[-2(1-i) Y_{1}^{7} Y_{2}+5 i Y_{1}^{4} Y_{2}^{4}+(1+i) Y_{1} Y_{2}^{7}\right] \\
3 i Y_{1}^{2} Y_{2}^{3}\left[2(1+i) Y_{1}^{3}+Y_{2}^{3}\right]
\end{array}\right), \\
Y_{\mathbf{3} I I}^{(8)} & \equiv\left(\begin{array}{c}
Y_{3 I I, 1}^{(8)} \\
Y_{3 I I, 2}^{(8)} \\
Y_{3 I I, 3}^{(8)}
\end{array}\right)=\left(\begin{array}{c}
3 e^{i \pi / 6} Y_{1}^{3} Y_{2}^{2}\left[Y_{1}^{3}-2(1-i) Y_{2}^{3}\right] \\
e^{i \pi / 3}\left[(1+i) Y_{1}^{7} Y_{2}-5 Y_{1}^{4} Y_{2}^{4}+2(1-i) Y_{1} Y_{2}^{7}\right] \\
-Y_{1}^{2}\left[Y_{1}^{6}-(1-i) Y_{1}^{3} Y_{2}^{3}+4 i Y_{2}^{6}\right]
\end{array}\right) .
\end{aligned}
$$




\section{APPENDIX C: NONMINIMAL KÄHLER POTENTIAL IN THE LEPTON SECTOR}

In the same manner as for quarks in Sec. VII, the minimal Kähler potential in the lepton sector is given by

$$
\begin{aligned}
\mathcal{K}_{\text {min }}= & (-i \tau+i \bar{\tau})^{-k_{L_{i}}} L_{i}^{\dagger} L_{i}+(-i \tau+i \bar{\tau})^{-k_{E_{i}^{c}}} E_{i}^{c \dagger} E_{i}^{c} \\
& +(-i \tau+i \bar{\tau})^{-k_{N_{i}^{c}}} N_{i}^{c \dagger} N_{i}^{c},
\end{aligned}
$$

where $L$ and $E^{c}$ denote lepton doublets and singlets, respectively, and $N^{c}$ stands for the right-handed neutrino superfield. Depending on the weight and representation assignments of $L, E^{c}$, and $N^{c}$, the most general form of the additional term $\Delta \mathcal{K}$ is given by

$$
\begin{aligned}
\Delta \mathcal{K}= & \sum_{k_{1}, k_{2}, \mathbf{r}_{1}, \mathbf{r}_{2}} \lambda_{L}^{i j}(-i \tau+i \bar{\tau})^{-k_{L_{i}}+k_{1}}\left(L_{i}^{\dagger} Y_{\mathbf{r}_{1}}^{\left(k_{1}\right) \dagger} Y_{\mathbf{r}_{2}}^{\left(k_{2}\right)} L_{j}\right)_{\mathbf{1}} \\
& +\sum_{k_{3}, k_{4}, \mathbf{r}_{1}, \mathbf{r}_{2}} \lambda_{E^{c}}^{i j}(-i \tau+i \bar{\tau})^{-k_{E_{i}^{c}}^{c}+k_{3}}\left(E_{i}^{c \dagger} Y_{\mathbf{r}_{1}}^{\left(k_{3}\right) \dagger} Y_{\mathbf{r}_{2}}^{\left(k_{4}\right)} E_{j}^{c}\right)_{\mathbf{1}} \\
& +\sum_{k_{5}, k_{6}, \mathbf{r}_{1}, \mathbf{r}_{2}} \lambda_{N^{c}}^{i j}(-i \tau+i \bar{\tau})^{-k_{N_{i}^{c}}+k_{5}}\left(N_{i}^{c \dagger} Y_{\mathbf{r}_{1}}^{\left(k_{5}\right) \dagger} Y_{\mathbf{r}_{2}}^{\left(k_{6}\right)} N_{j}^{c}\right)_{\mathbf{1}} \\
& + \text { H.c., }
\end{aligned}
$$

with

$k_{1}-k_{2}=k_{L_{i}}-k_{L_{j}}, \quad k_{3}-k_{4}=k_{E_{i}^{c}}-k_{E_{j}^{c}}$,

$k_{5}-k_{6}=k_{N_{i}^{c}}-k_{N_{j}^{c}}$.

The Hermitian and positive Kähler metrics for $L$, $E^{c}$, and $N^{c}$ can be easily read out and they can be written as

$$
\begin{aligned}
\mathcal{K}_{L} & =\Sigma_{L} D_{L}^{2} \Sigma_{L}^{\dagger}, \quad \mathcal{K}_{E^{c}}=\Sigma_{E^{c}} D_{E^{c}}^{2} \Sigma_{E^{c}}^{\dagger}, \\
\mathcal{K}_{N^{c}} & =\Sigma_{N^{c}} D_{N^{c}}^{2} \Sigma_{N^{c}}^{\dagger},
\end{aligned}
$$

where $\Sigma_{L, E^{c}, N^{c}}$ are unitary matrices, and $D_{L, E^{c}, N^{c}}$ are diagonal with positive real entries. The Kähler metrics as well as the kinetic terms can be diagonalized by the field redefinition

$$
\begin{aligned}
& L \rightarrow \hat{L}=H_{L}^{-1} L, \quad H_{L}=\Sigma_{L} D_{L}^{-1} \Sigma_{L}^{\dagger}, \\
& E^{c} \rightarrow \hat{E}^{c}=H_{E^{c}}^{-1} E^{c}, \quad H_{E^{c}}=\Sigma_{E^{c}} D_{E^{c}}^{-1} \Sigma_{E^{c}}^{\dagger}, \\
& N^{c} \rightarrow \hat{N}^{c}=H_{N^{c}}^{-1} N^{c}, \quad H_{N^{c}}=\Sigma_{N^{c}} D_{N^{c}}^{-1} \Sigma_{N^{c}}^{\dagger} .
\end{aligned}
$$

This field redefinition would lead to changes in the charged lepton and neutrino mass matrices. If neutrino masses are described by the effective Weinberg operator, we have

$$
\begin{aligned}
\mathcal{W}_{l} & =E^{c T} Y_{e} L H_{d}+\frac{1}{2 \Lambda} L^{T} Y_{\nu} L H_{u}^{2} \rightarrow \hat{\mathcal{W}}_{l} \\
& =\hat{E}^{c T} \hat{Y}_{e} \hat{L} H_{d}+\frac{1}{2 \Lambda} \hat{L}^{T} \hat{Y}_{\nu} \hat{L} H_{u}^{2},
\end{aligned}
$$

where $\hat{Y}_{e}=H_{E^{c}}^{T} Y_{e} H_{L}$ and $\hat{Y}_{\nu}=H_{L}^{T} Y_{\nu} H_{L}$. If neutrino masses are generated by the seesaw mechanism, going to the basis of canonically normalized fields, we obtain

$$
\begin{aligned}
\mathcal{W}_{l} & =E^{c T} Y_{e} L H_{d}+N^{c T} Y_{D} L H_{u}+\frac{1}{2} N^{c T} M_{N} N^{c} \\
& =\hat{E}^{c T} \hat{Y}_{e} \hat{L} H_{d}+\hat{N}^{c T} \hat{Y}_{D} \hat{L} H_{u}+\frac{1}{2} \hat{N}^{c T} \hat{M}_{N} \hat{N}^{c},
\end{aligned}
$$

with $\quad \hat{Y}_{e}=H_{E^{c}}^{T} Y_{e} H_{L}, \quad \hat{Y}_{D}=H_{N^{c}}^{T} Y_{D} H_{L}, \quad$ and $\quad \hat{M}_{N}=$ $H_{N^{c}}^{T} M_{N} H_{N^{c}}$. The charged lepton and neutrino mass eigenvalues and the lepton mixing matrix should be calculated for canonically normalized fields. In order to be more specific, we take the model $\mathcal{D}_{6}$ as an example. Given the field assignments in Table VIII, we can find the most general superpotential for $L$ which is a $T^{\prime}$ triplet with modular weight 1 ,

$$
\begin{aligned}
\mathcal{K}_{L}= & (-i \tau+i \bar{\tau})^{-1} L^{\dagger} L \\
& +\sum_{k_{1}, \mathbf{r}_{1}, \mathbf{r}_{2}}(-i \tau+i \bar{\tau})^{k_{1}-1}\left(L^{\dagger} Y_{\mathbf{r}_{1}}^{\left(k_{1}\right) \dagger} Y_{\mathbf{r}_{2}}^{\left(k_{1}\right)} L\right)_{\mathbf{1}}+\text { H.c. } \\
= & (-i \tau+i \bar{\tau})^{-1} L^{\dagger} L+\lambda_{1}\left(\left(L^{\dagger} Y_{\mathbf{2}}^{(1) \dagger}\right)_{\mathbf{2}^{\prime \prime}}\left(L Y_{\mathbf{2}}^{(1)}\right)_{\mathbf{2}}\right)_{\mathbf{1}} \\
& +\lambda_{2}\left(\left(L^{\dagger} Y_{\mathbf{2}}^{(1) \dagger}\right)_{\mathbf{2}^{\prime}}\left(L Y_{\mathbf{2}}^{(1)}\right)_{\mathbf{2}^{\prime}}\right)_{\mathbf{1}} \\
& +\lambda_{3}\left(\left(L^{\dagger} Y_{\mathbf{2}}^{(1) \dagger}\right)_{\mathbf{2}}\left(L Y_{\mathbf{2}}^{(1)}\right)_{\mathbf{2}^{\prime \prime}}\right)_{\mathbf{1}}+\ldots,
\end{aligned}
$$

where the couplings $\lambda_{1,2,3}$ are real. After field rescaling $L \rightarrow(-i \tau+i \bar{\tau})^{1 / 2} L$, the Kähler metric matrix is 


$$
\begin{aligned}
\mathcal{K}_{L}= & \left(\begin{array}{lll}
1 & 0 & 0 \\
0 & 1 & 0 \\
0 & 0 & 1
\end{array}\right)+\lambda_{1}(-i \tau+i \bar{\tau})\left(\begin{array}{ccc}
Y_{1}^{\dagger} Y_{1}+Y_{2}^{\dagger} Y_{2} & -\sqrt{2} e^{i \frac{7 \pi}{12}} Y_{1}^{\dagger} Y_{2} & -\sqrt{2} e^{i \frac{5 \pi}{12}} Y_{2}^{\dagger} Y_{1} \\
-\sqrt{2} e^{-i \frac{7 \pi}{12}} Y_{2}^{\dagger} Y_{1} & 2 Y_{2}^{\dagger} Y_{2} & 0 \\
-\sqrt{2} e^{-i \frac{5 \pi}{12}} Y_{1}^{\dagger} Y_{2} & 0 & 2 Y_{1}^{\dagger} Y_{1}
\end{array}\right) \\
& +\lambda_{2}(-i \tau+i \bar{\tau})\left(\begin{array}{ccc}
2 Y_{1}^{\dagger} Y_{1} & -\sqrt{2} e^{-i \frac{5 \pi}{12}} Y_{1}^{\dagger} Y_{2} & 0 \\
-\sqrt{2} e^{i \frac{5 \pi}{12}} Y_{2}^{\dagger} Y_{1} & Y_{1}^{\dagger} Y_{1}+Y_{2}^{\dagger} Y_{2} & -\sqrt{2} e^{i \frac{7 \pi}{12}} Y_{1}^{\dagger} Y_{2} \\
0 & -\sqrt{2} e^{-i \frac{7 \pi}{12}} Y_{2}^{\dagger} Y_{1} & 2 Y_{2}^{\dagger} Y_{2}
\end{array}\right) \\
& +\lambda_{3}(-i \tau+i \bar{\tau})\left(\begin{array}{ccc}
2 Y_{2}^{\dagger} Y_{2} & 0 & -\sqrt{2} e^{-i \frac{7 \pi}{12} Y_{2}^{\dagger} Y_{1}} \\
0 & 2 Y_{1}^{\dagger} Y_{1} & -\sqrt{2} e^{-i \frac{5 \pi}{12} Y_{1}^{\dagger} Y_{2}} \\
-\sqrt{2} e^{i \frac{i \pi}{12}} Y_{1}^{\dagger} Y_{2} & -\sqrt{2} e^{i \frac{5 \pi}{12}} Y_{2}^{\dagger} Y_{1} & Y_{1}^{\dagger} Y_{1}+Y_{2}^{\dagger} Y_{2}
\end{array}\right)+\ldots
\end{aligned}
$$

The right-handed neutrinos $N^{c}$ also transform as a triplet $N^{c} \sim \mathbf{3}$ under $T^{\prime}$ and carry modular weight 1 , and hence the Kähler potential and Kähler metric of $N^{c}$ are of similar form as Eqs. (C8) and (C9), respectively. The right-handed charged leptons are $T^{\prime}$ singlets $\mathbf{1}^{\prime}, \mathbf{1}^{\prime}$, and $\mathbf{1}$. The associated most general Kähler potential reads

$$
\begin{aligned}
\mathcal{K}_{E^{c}}= & (-i \tau+i \bar{\tau})^{-1} e^{c \dagger} e^{c}+(-i \tau+i \bar{\tau})^{-3} \mu^{c \dagger} \mu^{c} \\
& +(-i \tau+i \bar{\tau})^{-1} \tau^{c \dagger} \tau^{c} \\
& +\zeta_{1}(-i \tau+i \bar{\tau}) e^{c^{\dagger}} \tau^{c}\left(Y_{\mathbf{3}}^{(2) \dagger} Y_{\mathbf{3}}^{(2)}\right)_{\mathbf{1}^{\prime}} \\
& +\zeta_{1}^{*}(-i \tau+i \bar{\tau}) \tau^{c \dagger} e^{c}\left(Y_{\mathbf{3}}^{(2) \dagger} Y_{\mathbf{3}}^{(2)}\right)_{\mathbf{1}^{\prime \prime}} \\
& +\zeta_{2} e^{c^{\dagger}} \mu^{c}\left(Y_{\mathbf{2}}^{(1) \dagger} Y_{\mathbf{2}}^{(3)}\right)_{\mathbf{1}}+\zeta_{2}^{*} \mu^{c^{\dagger}} e^{c}\left(Y_{\mathbf{2}}^{(3) \dagger} Y_{\mathbf{2}}^{(1)}\right)_{\mathbf{1}} \\
& +\zeta_{3} \mu^{c \dagger} \tau^{c}\left(Y_{\mathbf{2}^{\prime \prime}}^{(3) \dagger} Y_{\mathbf{2}}^{(1)}\right)_{\mathbf{1}^{\prime}}+\zeta_{3}^{*} \tau^{c \dagger} \mu^{c}\left(Y_{\mathbf{2}}^{(1) \dagger} Y_{\mathbf{2}^{\prime \prime}}^{(3)}\right)_{\mathbf{1}^{\prime \prime}}+\ldots
\end{aligned}
$$

We plot the dependence of the lepton mixing angles, mass ratio $m_{r} \equiv \sqrt{\Delta m_{21}^{2} / \Delta m_{31}^{2}}$, and $\delta_{C P}^{l}$ on the parameter $\lambda_{3}$ in Fig. 2, where the values of other input parameters in the model $\mathcal{D}_{6}$ are adapted from Table XI. We see that the corrections from the Kähler potential can be sizable, which confirms the insight of Ref. [30]. This drawback of bottomup construction with modular symmetry can be overcame by combining the traditional and modular flavor symmetries [76]. In this scheme, both the superpotential and Kähler potential are strongly constrained, and the offdiagonal contributions to the Kähler metric are forbidden by all symmetries of the theory. Consequently, the additional terms depending on modular forms in the Kähler potential do not significantly change the phenomenological predictions obtained by assuming a minimal Kähler potential.
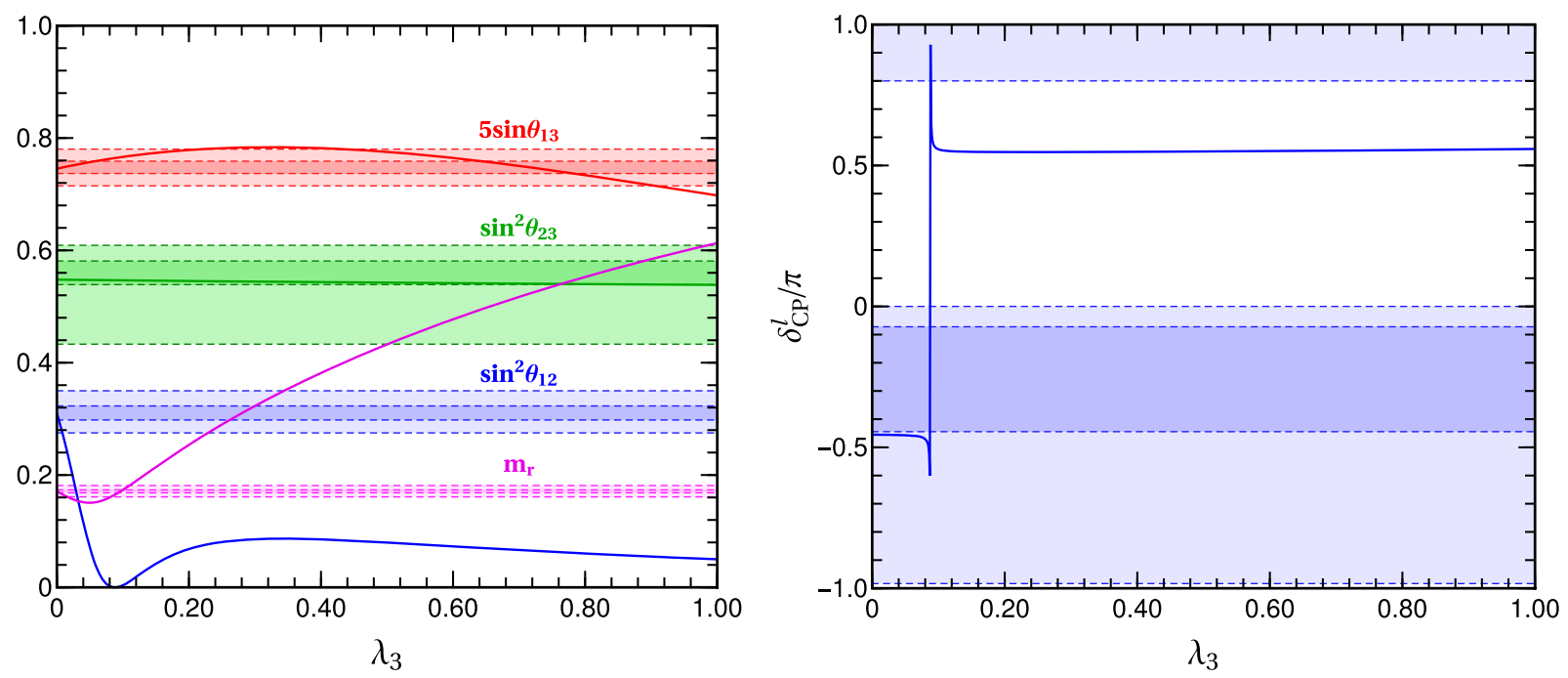

FIG. 2. Variation of the lepton mixing angles, mass ratio $m_{r} \equiv \sqrt{\Delta m_{21}^{2} / \Delta m_{31}^{2}}$ (left panel), and Dirac $C P$ phase $\delta_{C P}^{l}$ (right panel) with respect to the coupling $\lambda_{3}$. The shaded regions represent the $1 \sigma$ and $3 \sigma$ ranges of the mixing parameters [1]. 
[1] I. Esteban, M. C. Gonzalez-Garcia, A. HernandezCabezudo, M. Maltoni, and T. Schwetz, Global analysis of three-flavour neutrino oscillations: Synergies and tensions in the determination of $\theta_{23}, \delta_{C P}$, and the mass ordering, J. High Energy Phys. 01 (2019) 106.

[2] K. Abe et al. (T2K Collaboration), Measurement of neutrino and antineutrino oscillations by the $\mathrm{T} 2 \mathrm{~K}$ experiment including a new additional sample of $\nu_{e}$ interactions at the far detector, Phys. Rev. D 96, 092006 (2017); Erratum, Phys. Rev. D 98, 019902 (2018).

[3] K. Abe et al. (T2K Collaboration), Search for $C P$ Violation in Neutrino and Antineutrino Oscillations by the T2K Experiment with $2.2 \times 10^{21}$ Protons on Target, Phys. Rev. Lett. 121, 171802 (2018).

[4] G. Altarelli and F. Feruglio, Discrete flavor symmetries and models of neutrino mixing, Rev. Mod. Phys. 82, 2701 (2010).

[5] H. Ishimori, T. Kobayashi, H. Ohki, Y. Shimizu, H. Okada, and M. Tanimoto, Non-Abelian discrete symmetries in particle physics, Prog. Theor. Phys. Suppl. 183, 1 (2010).

[6] S. F. King and C. Luhn, Neutrino mass and mixing with discrete symmetry, Rep. Prog. Phys. 76, 056201 (2013).

[7] S. F. King, A. Merle, S. Morisi, Y. Shimizu, and M. Tanimoto, Neutrino mass and mixing: From theory to experiment, New J. Phys. 16, 045018 (2014).

[8] S. F. King, Models of neutrino mass, mixing and $C P$ violation, J. Phys. G 42, 123001 (2015).

[9] S. F. King, Unified models of neutrinos, flavour and $C P$ violation, Prog. Part. Nucl. Phys. 94, 217 (2017).

[10] F. Feruglio and A. Romanino, Neutrino flavour symmetries, arXiv: 1912.06028.

[11] F. Feruglio, Are neutrino masses modular forms? in From My Vast Repertoire ...: Guido Altarelli's Legacy, edited by A. Levy, S. Forte, and G. Ridolfi (World Scientific, Singapore, 2019), pp. 227-266, https://doi.org/10.1142/ 9789813238053_0012.

[12] L. J. Dixon, D. Friedan, E. J. Martinec, and S. H. Shenker, The conformal field theory of orbifolds, Nucl. Phys. B282, 13 (1987).

[13] S. Hamidi and C. Vafa, Interactions on orbifolds, Nucl. Phys. B279, 465 (1987).

[14] J. Lauer, J. Mas, and H. P. Nilles, Duality and the role of nonperturbative effects on the world sheet, Phys. Lett. B 226, 251 (1989).

[15] D. Cremades, L. E. Ibanez, and F. Marchesano, Yukawa couplings in intersecting $D$-brane models, J. High Energy Phys. 07 (2003) 038.

[16] R. Blumenhagen, M. Cvetic, P. Langacker, and G. Shiu, Toward realistic intersecting $D$-brane models, Annu. Rev. Nucl. Part. Sci. 55, 71 (2005).

[17] D. Cremades, L. E. Ibanez, and F. Marchesano, Computing Yukawa couplings from magnetized extra dimensions, J. High Energy Phys. 05 (2004) 079.

[18] H. Abe, K.-S. Choi, T. Kobayashi, and H. Ohki, NonAbelian discrete flavor symmetries from Magnetized/ Intersecting brane models, Nucl. Phys. B820, 317 (2009).

[19] L. E. Ibanez, Hierarchy of quark-Lepton masses in orbifold superstring compactification, Phys. Lett. B 181, 269 (1986).

[20] O. Lebedev, The CKM phase in heterotic orbifold models, Phys. Lett. B 521, 71 (2001).
[21] P. Brax and M. Chemtob, Flavor changing neutral current constraints on standard-like orbifold models, Phys. Rev. D 51, 6550 (1995).

[22] P. Binetruy and E. Dudas, Dynamical mass matrices from effective superstring theories, Nucl. Phys. B451, 31 (1995).

[23] E. Dudas, S. Pokorski, and C. A. Savoy, Soft scalar masses in supergravity with horizontal U(1)-x gauge symmetry, Phys. Lett. B 369, 255 (1996).

[24] P. P. Novichkov, J. T. Penedo, S. T. Petcov, and A. V. Titov, Generalized $C P$ symmetry in modular-invariant models of flavour, J. High Energy Phys. 07 (2019) 165.

[25] A. Baur, H. P. Nilles, A. Trautner, and P. K. S. Vaudrevange, Unification of flavor, $C P$, and modular symmetries, Phys. Lett. B 795, 7 (2019).

[26] B. S. Acharya, D. Bailin, A. Love, W. A. Sabra, and S. Thomas, Spontaneous breaking of $C P$ symmetry by orbifold moduli, Phys. Lett. B 357, 387 (1995); Erratum, Phys. Lett. B 407, 451 (1997).

[27] T. Dent, $C P$ violation and modular symmetries, Phys. Rev. D 64, 056005 (2001).

[28] J. Giedt, $C P$ violation and moduli stabilization in heterotic models, Mod. Phys. Lett. A 17, 1465 (2002).

[29] A. Baur, H. P. Nilles, A. Trautner, and P. K. S. Vaudrevange, A string theory of flavor and $C P$, Nucl. Phys. B947, 114737 (2019).

[30] M.-C. Chen, S. Ramos-Sanchez, and M. Ratz, A note on the predictions of models with modular flavor symmetries, Phys. Lett. B 801, 135153 (2020).

[31] T. Kobayashi, K. Tanaka, and T. H. Tatsuishi, Neutrino mixing from finite modular groups, Phys. Rev. D 98, 016004 (2018).

[32] T. Kobayashi, Y. Shimizu, K. Takagi, M. Tanimoto, T. H. Tatsuishi, and H. Uchida, Finite modular subgroups for fermion mass matrices and baryon/lepton number violation, Phys. Lett. B 794, 114 (2019).

[33] T. Kobayashi, Y. Shimizu, K. Takagi, M. Tanimoto, and T. H. Tatsuishi, Modular $S_{3}$ invariant flavor model in SU(5) GUT, arXiv:1906.10341.

[34] H. Okada and Y. Orikasa, A modular $S_{3}$ symmetric radiative seesaw model, Phys. Rev. D 100, 115037 (2019).

[35] J. C. Criado and F. Feruglio, Modular invariance faces precision neutrino data, SciPost Phys. 5, 042 (2018).

[36] T. Kobayashi, N. Omoto, Y. Shimizu, K. Takagi, M. Tanimoto, and T. H. Tatsuishi, Modular $A_{4}$ invariance and neutrino mixing, J. High Energy Phys. 11 (2018) 196.

[37] F. J. de Anda, S. F. King, and E. Perdomo, $S U(5)$ grand unified theory with $A_{4}$ modular symmetry, Phys. Rev. D 101, 015028 (2020).

[38] H. Okada and M. Tanimoto, $C P$ violation of quarks in $A_{4}$ modular invariance, Phys. Lett. B 791, 54 (2019).

[39] P. P. Novichkov, S. T. Petcov, and M. Tanimoto, Trimaximal neutrino mixing from modular $\mathrm{A}_{4}$ invariance with residual symmetries, Phys. Lett. B 793, 247 (2019).

[40] T. Nomura and H. Okada, A modular $A_{4}$ symmetric model of dark matter and neutrino, Phys. Lett. B 797, 134799 (2019).

[41] H. Okada and M. Tanimoto, Towards unification of quark and lepton flavors in $A_{4}$ modular invariance, arXiv:1905 .13421 . 
[42] T. Nomura and H. Okada, A two loop induced neutrino mass model with modular $A_{4}$ symmetry, arXiv:1906.03927.

[43] G.-J. Ding, S. F. King, and X.-G. Liu, Modular A A $_{4}$ symmetry models of neutrinos and charged leptons, J. High Energy Phys. 09 (2019) 074.

[44] H. Okada and Y. Orikasa, A radiative seesaw model in modular $A_{4}$ symmetry, arXiv:1907.13520.

[45] T. Nomura, H. Okada, and O. Popov, A modular $A_{4}$ symmetric scotogenic model, Phys. Lett. B 803, 135294 (2020).

[46] T. Kobayashi, Y. Shimizu, K. Takagi, M. Tanimoto, and T. H. Tatsuishi, $A_{4}$ lepton flavor model and modulus stabilization from $S_{4}$ modular symmetry, Phys. Rev. D 100, 115045 (2019).

[47] T. Asaka, Y. Heo, T. H. Tatsuishi, and T. Yoshida, Modular $A_{4}$ invariance and leptogenesis, J. High Energy Phys. 01 (2020) 144.

[48] G.-J. Ding, S. F. King, X.-G. Liu, and J.-N. Lu, Modular $S_{4}$ and $A_{4}$ symmetries and their fixed points: New predictive examples of lepton mixing, J. High Energy Phys. 12 (2019) 030 .

[49] D. Zhang, A modular $A_{4}$ symmetry realization of two-zero textures of the Majorana neutrino mass matrix, Nucl. Phys. B952, 114935 (2020).

[50] T. Nomura, H. Okada, and S. Patra, An Inverse Seesaw model with $A_{4}$-modular symmetry, arXiv:1912.00379.

[51] T. Kobayashi, T. Nomura, and T. Shimomura, Type II seesaw models with modular $A_{4}$ symmetry, arXiv:1912 .00637 .

[52] J. T. Penedo and S. T. Petcov, Lepton masses and mixing from modular $S_{4}$ symmetry, Nucl. Phys. B939, 292 (2019).

[53] P. P. Novichkov, J. T. Penedo, S. T. Petcov, and A. V. Titov, Modular $\mathrm{S}_{4}$ models of lepton masses and mixing, J. High Energy Phys. 04 (2019) 005.

[54] I. de Medeiros Varzielas, S. F. King, and Y.-L. Zhou, Multiple modular symmetries as the origin of flavour, Phys. Rev. D 101, 055033 (2020).

[55] T. Kobayashi, Y. Shimizu, K. Takagi, M. Tanimoto, and T. H. Tatsuishi, New $A_{4}$ lepton flavor model from $S_{4}$ modular symmetry, J. High Energy Phys. 02 (2020) 097.

[56] S. F. King and Y.-L. Zhou, Trimaximal $\mathrm{TM}_{1}$ mixing with two modular $S_{4}$ groups, Phys. Rev. D 101, 015001 (2020).

[57] J. C. Criado, F. Feruglio, F. Feruglio, and S. J. D. King, Modular invariant models of lepton masses at levels 4 and 5, J. High Energy Phys. 02 (2020) 001.

[58] X. Wang and S. Zhou, The minimal seesaw model with a modular $S_{4}$ symmetry, J. High Energy Phys. 05 (2020) 017.

[59] P. P. Novichkov, J. T. Penedo, S. T. Petcov, and A. V. Titov, Modular $A_{5}$ symmetry for flavour model building, J. High Energy Phys. 04 (2019) 174.

[60] G.-J. Ding, S. F. King, and X.-G. Liu, Neutrino mass and mixing with $A_{5}$ modular symmetry, Phys. Rev. D 100, 115005 (2019).

[61] X.-G. Liu and G.-J. Ding, Neutrino masses and mixing from double covering of finite modular groups, J. High Energy Phys. 08 (2019) 134.

[62] H. Fritzsch, Calculating the cabibbo angle, Phys. Lett. 70B, 436 (1977).
[63] S. Weinberg, The problem of mass, Trans. N.Y. Acad. Sci. 38, 185 (1977).

[64] F. Wilczek and A. Zee, Discrete flavor symmetries and a formula for the cabibbo angle, Phys. Lett. 70B, 418 (1977); Erratum, Phys. Lett. 72B, 504 (1978).

[65] W. Grimus, A. S. Joshipura, L. Lavoura, and M. Tanimoto, Symmetry realization of texture zeros, Eur. Phys. J. C 36, 227 (2004).

[66] R. G. Felipe and H. Serôdio, Abelian realization of phenomenological two-zero neutrino textures, Nucl. Phys. B886, 75 (2014).

[67] P. O. Ludl and W. Grimus, A complete survey of texture zeros in the lepton mass matrices, J. High Energy Phys. 07 (2014) 090; Erratum, J. High Energy Phys. 10 (2014) 126.

[68] P. O. Ludl and W. Grimus, A complete survey of texture zeros in general and symmetric quark mass matrices, Phys. Lett. B 744, 38 (2015).

[69] H. Fritzsch and Z.-z. Xing, Mass and flavor mixing schemes of quarks and leptons, Prog. Part. Nucl. Phys. 45, 1 (2000).

[70] M. Gupta and G. Ahuja, Flavor mixings and textures of the fermion mass matrices, Int. J. Mod. Phys. A 27, 1230033 (2012).

[71] R. de Adelhart Toorop, F. Feruglio, and C. Hagedorn, Finite modular groups and lepton mixing, Nucl. Phys. B858, 437 (2012).

[72] S. Ferrara, D. Lust, A. D. Shapere, and S. Theisen, Modular invariance in supersymmetric field theories, Phys. Lett. B 225, 363 (1989).

[73] S. Ferrara, D. Lust, and S. Theisen, Target space modular invariance and low-energy couplings in orbifold compactifications, Phys. Lett. B 233, 147 (1989).

[74] L. J. Dixon, V. Kaplunovsky, and J. Louis, On effective field theories describing $(2,2)$ vacua of the heterotic string, Nucl. Phys. B329, 27 (1990).

[75] H. P. Nilles, S. Ramos-Sanchez, and P. K. S. Vaudrevange, Eclectic flavor groups, J. High Energy Phys. 02 (2020) 045.

[76] H. P. Nilles, S. Ramos-Sanchez, and P. K. Vaudrevange, Lessons from eclectic flavor symmetries, arXiv:2004 .05200 .

[77] S. Antusch and V. Maurer, Running quark and lepton parameters at various scales, J. High Energy Phys. 11 (2013) 115.

[78] S. Ferrara, N. Magnoli, T. R. Taylor, and G. Veneziano, Duality and supersymmetry breaking in string theory, Phys. Lett. B 245, 409 (1990).

[79] M. Cvetic, A. Font, L. E. Ibanez, D. Lust, and F. Quevedo, Target space duality, supersymmetry breaking and the stability of classical string vacua, Nucl. Phys. B361, 194 (1991).

[80] M.-C. Chen, M. Fallbacher, M. Ratz, and C. Staudt, On predictions from spontaneously broken flavor symmetries, Phys. Lett. B 718, 516 (2012).

[81] M.-C. Chen, M. Fallbacher, Y. Omura, M. Ratz, and C. Staudt, Predictivity of models with spontaneously broken non-Abelian discrete flavor symmetries, Nucl. Phys. B873, 343 (2013). 